Aus der Abteilung Psychiatrie und Psychotherapie

(ehem. Leiter: Prof. Dr. med. E. Rüther)

im Zentrum Psychosoziale Medizin

der Medizinischen Fakultät der Universität Göttingen

\title{
Der Schlaf im Verlauf einer Behandlung mit Interpersoneller Psychotherapie im Vergleich mit progressiver Muskelrelaxation von Patienten mit primärer Insomnie
}

\author{
INAUGURAL-DISSERTATION \\ zur Erlangung des Doktorgrades \\ der Medizinischen Fakultät \\ der Georg-August-Universität zu Göttingen \\ vorgelegt von \\ Christine Meyer \\ aus \\ Bremerhaven
}

Göttingen 2009 
Dekan: Prof. Dr. med. C. Frömmel

I. Berichterstatter: Prof. Dr. med. G. Hajak

II. Berichterstatter/in: Prof. Dr. med. C. Hermann-Lingen

III. Berichterstatter/in:

Tag der mündlichen Prüfung: 26.09.2011 


\section{Inhaltsverzeichnis}

\section{ABBILDUNGSVERZEICHNIS}

1.1

Primäre Insomnie

1.1.1 Definition

1.1.2 Epidemiologie

1.1.3 Besonderheiten des Schlafes und seiner Behandlung im Alter

1.1 .4

Pathogenese

1.1.5

Symptome

1.1.5.1 Wahrnehmung und Fehlwahrnehmung des Schlafes

1.1.6 Diagnostik der Insomnie

1.1.7 Behandlung der Insomnie

$\begin{array}{lll}\text { 1.1.7.1 Medikamentöse Therapieformen } & 7\end{array}$

1.1.7.2 Nichtmedikamentöse Therapieformen 9

1.1.7.2.1 Grundlegende Maßnahmen: Aufklärung, Beratung und Schlafhygiene 10

1.1.7.2.2 Entspannungsverfahren 11

1.1.7.2.3 Verhaltenstherapeutische, kognitive und regulierende Techniken 12

1.1.7.2.4 Psychotherapie im engeren Sinne 14

$\begin{array}{lll}\text { 1.1.7.2.4.1 Behandlungsdauer } & 15\end{array}$

$\begin{array}{lll}\text { 1.1.7.2.5 Vergleich } & 16\end{array}$

$\begin{array}{llr}1.2 & 18\end{array}$

$\begin{array}{lll}\text { 1.2.1 Einleitung } & 18\end{array}$

$\begin{array}{ll}1.2 .2 & 19\end{array}$

$\begin{array}{lll}1.2 .3 & 20\end{array}$

1.2.3.1 Die Bearbeitung des Problembereichs 24

$\begin{array}{lll}\text { 1.2.3.1.1 Trauer } & 24\end{array}$

1.2.3.1.2 Interpersonelle Rollenkonflikte 25

1.2.3.1.3 Rollenwechsel 26

1.2.3.1.4 Interpersonelle Defizite 26

1.2.3.2 Beendigung der Therapie $\quad 27$

$\begin{array}{lll}\text { 1.2.4 Die Effektivität der IPT und ihrer Modifikationen } & 27\end{array}$

1.3 Progressive Muskelrelaxation $\quad 29$

$\begin{array}{lll}\text { 1.3.1 Die Durchführung der PMR } & 30\end{array}$

1.3.2 Effektivität der PMR bei Patienten mit primärer Insomnie 32 
1.4 Das Ziel dieser Untersuchung

1.4.1 Annahmen über die Veränderungen des subjektiven Schlafes und ihre Konsequenzen für die Auswertung $\quad 35$

1.4.1.1 Schlafquantität $\quad 35$

1.4.1.2 Schlafqualität

1.4.1.3 Bewertungszeitraum 35

1.4.1.3.1 Die Bewertung des Schlafes der zurückliegenden zwei Wochen 35

1.4.1.3.2 Der Schlaf in der täglichen Bewertung 36

1.4.1.4 Der Schlaf nach zwei Dritteln des Behandlungszeitraumes 36

$2 \quad$ MATERIAL UND METHODEN 37

$\begin{array}{llr}2.1 & \text { Patienten } & 37\end{array}$

$\begin{array}{llr}2.2 & \text { Studienaufbau } & 39\end{array}$

$2.3 \quad$ Untersuchungsverfahren $\quad 41$

2.3.1 Strukturiertes Interview für Schlafstörungen (SIS-D) 41

2.3.2 Polysomnografie 41

2.3.3 Schlaffragebögen A und B (SF-A und SF-B) 43

2.3.4 Visuelle Analogskalen zur Erfassung der Schlafqualität (VIS-M) 43

$\begin{array}{lll}2.3 .5 & \text { Urinkontrollen }\end{array}$

$2.4 \quad$ Therapeutische Verfahren $\quad 45$

2.4.1 Interpersonelle Psychotherapie für Insomniepatienten 45

2.4.2 Progressive Muskelrelaxation 46

$\begin{array}{llr}2.5 & \text { Auswertung der Daten } & 46\end{array}$

$3 \quad$ ERGEBNISSE $\quad 52$

3.1 Patienten - Stichprobe $\quad 52$

Subjektive Schlafparameter vor und nach der Behandlung 53

3.2.1 Die subjektive Bewertung des Schlafes im Rückblick auf die vergangenen zwei Wochen (SF-B) 53

3.2.1.1 SF-B - quantitative Parameter $\quad 53$

3.2.1.2 SF-B - qualitative Parameter $\quad 57$

3.2.2 Die subjektive Bewertung des Schlafes im Rückblick auf die vergangene Nacht (SF-A) 62

3.2.2.1 SF-A - quantitative Parameter 62

$\begin{array}{lll}\text { 3.2.2.2 } & \text { SF-A - qualitative Parameter } & 67\end{array}$ 
4.4.1.1 Die Schlafbewertung eines zweiwöchigen Zeitraumes 93

4.4.1.1.1 quantitative Parameter 93

4.4.1.1.2 qualitative Parameter 94

4.4.1.2 Der Schlaf in der Bewertung der vergangenen Nacht 95

4.4.1.2.1 quantitative Parameter 95

$\begin{array}{lll}\text { 4.4.1.2.2 } & 97\end{array}$

$\begin{array}{lll}\text { 4.4.2 objektive Schlafparameter } & 98\end{array}$

4.4.2.1 Die Auswertung polysomnografischer Ableitungen 98

4.4.2.2 Gegenüberstellung von objektiven und subjektiven Schlafparametern

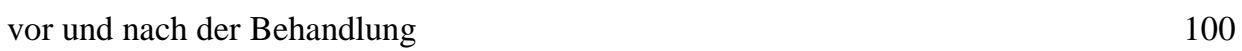

4.4.3 Verlaufsbetrachtung anhand subjektiver Schlafparameter (VIS-M) 101

4.4.3.1 Quantitative Parameter 102

4.4.3.2 Qualitative Parameter 103

4.4.4 Verlaufsbetrachtung anhand subjektiver Schlafparameter (VIS-M) mit Berücksichtigung der Baseline und der Daten aus dem Schlaflabor $\quad 104$

4.4.5 Veränderungen in den ersten zwei Dritteln des Behandlungszeitraums 107

$\begin{array}{llr}4.5 & \text { Die Therapeutin } & 108\end{array}$

$\begin{array}{llr}4.6 & \text { Zusammenfassende Beurteilung und Ausblick } & 108\end{array}$

$5 \quad$ ZUSAMMENFASSUNG $\quad 111$

$\begin{array}{llr}6 & \text { ANHANG } & 113\end{array}$

$\begin{array}{lll}7 & \text { LITERATURVERZEICHNIS } & 115\end{array}$ 


\section{Abbildungsverzeichnis}

Abb. 2.01: Auswertung der Daten

Abb. 2.02: Auswertung der zu den Polysomnografieterminen erhobenen Daten des SF-A und des SF-B 49

Abb. 2.03: VIS-M-Verlaufsbetrachtung 50

Abb. 3.01: SF-B-Schlafparameter: TST, arithmetische Mittelwerte zum Zeitpunkt der Eingangs- (EP) und Kontrollpolysomnografie (KP) 53

Abb. 3.02: SF-B-Schlafparameter: WASO, arithmetische Mittelwerte zum Zeitpunkt der Eingangs- (EP) und Kontrollpolysomnografie (KP) 54

Abb. 3.03: SF-B-Schlafparameter: SQ, arithmetische Mittelwerte zum Zeitpunkt der Eingangs- (EP) und Kontrollpolysomnografie (KP) 58

Abb. 3.04: SF-B-Schlafparameter: $\mathrm{mE}$, arithmetische Mittelwerte zum Zeitpunkt der Eingangs- (EP) und Kontrollpolysomnografie (KP)

Abb. 3.05: SF-A-Schlafparameter: TST, arithmetische Mittelwerte zum Zeitpunkt der Eingangs- (EP) und Kontrollpolysomnografie (KP)

Abb. 3.06: SF-A-Schlafparameter: WASO, arithmetische Mittelwerte zum Zeitpunkt der Eingangs- (EP) und Kontrollpolysomnografie (KP) 64

Abb. 3.07: SF-A-Schlafparameter: SQ, arithmetische Mittelwerte zum Zeitpunkt der Eingangs- (EP) und Kontrollpolysomnografie (KP) 68

Abb. 3.08: SF-A-Schlafparameter: $\mathrm{mE}$, arithmetische Mittelwerte zum Zeitpunkt der Eingangs- (EP) und Kontrollpolysomnografie (KP) 70

Abb. 3.09: 1. Verlaufsbetrachtung VIS-M: tatsächliche Schlafzeit (TST) in Minuten __ 76

Abb. 3.10: 2. Verlaufsbetrachtung VIS-M: Einschlaflatenz (EL) in Minuten___ 77

Abb. 3.11: 3. Verlaufsbetrachtung VIS-M: Schlafqualität (SQ) _ 78

Abb. 3.12: 4. Verlaufsbetrachtung VIS-M: morgendliches Erholungsgefühl (mE)__ 79

Abb. 3.13: 1. eingebettete Verlaufsbetrachtung VIS-M: tatsächliche Schlafzeit (TST) in Minuten _ 86

Abb. 3.14: 2. eingebettete Verlaufsbetrachtung VIS-M: Einschlaflatenz (EL) in Minuten___ 87

Abb. 3.15: 3. eingebettete Verlaufsbetrachtung VIS-M: Schlafqualität (SQ) ___ 88

Abb. 3.16: 4. eingebettete Verlaufsbetrachtung VIS-M: morgendliches Erholungsgefühl (mE)__ 89 


\section{Einleitung}

\subsection{Primäre Insomnie}

\subsubsection{Definition}

Mit dem Wort „Schlaflosigkeit“ werden Schlafstörungen bezeichnet, die aus dem Missverhältnis von Schlafbedürfnis und Schlafvermögen entstehen und sich in einem Mangel an Schlafqualität und Schlafquantität (Hajak und Rüther 1995) äußern. Wegen objektiver oder auch nur subjektiv wahrgenommener Ein- oder Durchschlafstörungen wird der Schlaf als derart ungenügend oder nicht erholsam empfunden, dass erheblicher Leidensdruck und / oder eine Einschränkung der Tagesbefindlichkeit bzw. eine störende Auswirkung auf die alltägliche Funktionsfähigkeit (WHO 2008) die Folgen sind. Tritt diese Schlaflosigkeit mindestens dreimal pro Woche über einen Zeitraum von wenigstens einem Monat auf, so sind nach ICD-10 (WHO 1991, WHO 2008) die Kriterien für eine Insomnie erfüllt. In der Definition nach DSM-IV (APA 1996) ist keine Mindestfreqenz pro Woche angegeben.

Einige Autoren verstehen unter Insomnie keine Krankheit, sondern ein Symptom, das abgeklärt werden muss (Hauri und Esther 1990, Dement et al. 1984). Liegt der Insomnie, auf die der Patient oft sehr fixiert ist, keine organische Ursache zugrunde und ist sie nicht Teil einer anderen Schlaf- oder psychischen Störung, so handelt es sich um eine primäre (synonym: psychophysiologische, nichtorganische) Insomnie (APA 1987, Hauri 1989, WHO 2008). Praktisch kann es jedoch schwierig sein, zwischen den beiden häufigsten DSM-IV-Diagnosen „primäre Insomnie“ und „Insomnie im Rahmen einer psychischen Störung“ zu differenzieren (Nowell et al. 1997a). Die erstgenannte ist mit einem erhöhten Risiko verbunden, vor allem an Depression, jedoch auch an einer Angststörung und an Alkoholismus zu erkranken (Weissman et al. 1997, Gillin 1998).

\subsubsection{Epidemiologie}

Ein Viertel der westdeutschen Bevölkerung leidet an Ein- bzw Durchschlafstörungen, die nicht durch äußere Einflüsse bedingt sind, doch nur jeder zweite chronisch, das heißt länger als zwei Jahre, davon Betroffene konsultiert deshalb einen Arzt (Simen et al. 1995). 
Schlafbeschwerden treten mit zunehmendem Alter, niedrigem Sozialstatus und Bildungsniveau und bei Arbeitslosigkeit häufiger auf, Frauen sind besonders betroffen. (Holzrichter et al. 1994, Hajak und Rüther 1991a, Mellinger et al. 1985, Ford und Kamerow 1989). Frauen könnten unter anderem aufgrund des hormonellen Einflusses auf das Schlaf-Wach-Verhalten und die Stimmung ein erhöhtes Risiko aufweisen, im Laufe des Lebenszyklus an Insomnie zu erkranken (Soares und Murray 2006). Ein bedeutender genetischer Einfluss auf den Schlaf zeigte sich in Zwillingsstudien in Finnland und Australien (Partinen et al. 1983, Heath et al. 1990). Hierbei kann ein Polymorphismus in der Promotorregion des Monoaminoxinase-A-Gens, der zu einem Allel mit verminderter Transkriptioneffizienz führt, eine Rolle spielen (Brummett et al. 2007).

\subsubsection{Besonderheiten des Schlafes und seiner Behandlung im Alter}

Einschlaflatenz, nächtliches Erwachen, Schlaffragmentierung und Nickerchen am Tage nehmen im Alter signifikant zu, Schlafzeit und Schlafeffizienz dagegen ab, wie mittels Aktigrafie der Handgelenksaktivität gemessen wurde (Huang et al. 2002). Die Häufigkeit von nächtlichen Aufwachvorgängen nimmt im Alter zu. Ursächlich können folgende Fakten sein: Altersbedingt kommt es zu einer Reduktion der SchlafKonsolidierung und zu einer Reduktion der Konsolidierung des Non-REM-Schlafes, die physiologische REM-Schlaf-Zunahme gegen Ende der Nacht nimmt im Alter ab (Dijk, Duffy and Czeisler 2001). Evidenzbasierte psychologische Behandlungsformen für die Insomnie im Alter sind nach den Kriterien des American Psychological Association's Committee on Science and Practice of the Society for Clinical Psychology die Schlafrestriktions-/Schlafkompressionstherapie und die Multikomponenten-kognitivbehaviorale Therapie, z.T. auch die Stimuluskontrolle; die für die Untersuchung angesetzte Altersgrenze $>=60$ Jahre und der Mangel an konsequenter Beachtung von Komorbiditäten wird jedoch von den Autoren kritisiert (Mc Curry et al. 2007). Bei Älteren können atemstimulierende Einreibungen Entspannung und Schlaf fördern und werden zudem als sehr angenehm empfunden (Schiff 2006). Yoga kann bei älteren signifikant die Einschlaflatenz reduzieren und sowohl die Gesamtschlafzeit als auch das Gefühlt des Ausgeruhtseins am Morgen steigern (Manjunath und Telles 2005).

\subsubsection{Pathogenese}

Einige Studien haben belegt, dass einschneidende Lebensereignisse, mangelnde soziale Kompetenz und die Neigung, Emotionen $\mathrm{zu}$ internalisieren, für Insomnie prädisponierende Faktoren darstellen, andere konnten mittels Clusteranalysen 
unterschiedliche Persönlichkeitsprofile von Insomniepatienten abgrenzen (HerrmannMaurer et al. 1988).

Die Studien zeigten, dass Insomniepatienten im Jahr des Beschwerdebeginns vermehrt mit einschneidenden Lebensereignissen konfrontiert wurden, die zumeist negativ und mit Krankheit oder Verlust im sozialen Umfeld verbunden waren. Diese Begebenheiten wirken als Stressoren auf Menschen, die aufgrund ihrer erhöhten persönlichen Verwundbarkeit mit der Schlafstörung reagieren. Diese Patienten waren schon als Kinder eher verstimmt und unzufriedener mit ihren Eltern und dem Familienleben; sie neigten schon damals dazu, ihre Unzufriedenheit zu internalisieren und zu somatisieren, was sich in Ess- und Schlafproblemen zeigte (Healey et al. 1981). Diese Tendenz, Gefühle zu internalisieren anstatt sie offen auszudrücken (Kales et al. 1976, Coursey et al. 1975), führt zur Erregung. Das emotionale Arousal zieht eine physiologische Aktivierung nach sich, die nicht mit dem Schlaf vereinbar ist: Sowohl Ein- als auch Durchschlafstörungen sind die Folge (Kales et al. 1984). Akuter psychophysiologischer Stress ist mit abnehmender parasympathischer Modulation während Non-REM- und REM-Schlaf vergesellschaftet, mit zunehmender sympathovagaler Balance während des Non-REM-Schlafes, mit schlechterem Durchschlafen und verminderter Delta-Aktivität (Hall et al. 2004).

Insomniepatienten schlossen und unterhielten vor dem Auftreten der Schlafstörung weniger Freundschaften und fühlten sich häufiger unterlegen. Das Selbstbild der Betroffenen ist deutlich negativer als das guter Schläfer: sie sehen sich selbst eher als aggressiv, passiv, schwach, ungenügend, unkontrolliert, weniger anpassungsfähig und töricht; außerdem haben sie mehr Schwierigkeiten in interpersonellen Beziehungen (Healey et al. 1981). Andere Autoren charakterisieren Insomniepatienten als „angespannte und ängstliche Personen, die über die Probleme des Tages grübeln, während sie versuchen einzuschlafen“ (Kales und Kales 1984, S.94). Ihre zahlreichen psychosozialen Probleme (Kales et al. 1984) finden Ausdruck in sozialem Rückzug, gesteigerter Selbstbezogenheit und körperlicher und geistiger Inaktivität während des Tages, die möglicherweise mit nächtlicher Hyperaktivität einhergeht (Marchini et al. 1983). Von erhöhter Wachsamkeit und gesteigerter Aufmerksamkeit auch während des Tages wird ebenfalls berichtet (Regestein et al. 1993). Schlechte Schläfer neigen dazu, bei zweideutig zu interpretierenden Szenarien eher eine bedrohliche Entwicklung anzunehmen als gute Schläfer (Ree, Pollitt und Harvey 2006).

Zwei Gruppen von Insomniepatienten konnte eine Forschergruppe nach Erstmanifestationsalter differenzieren, von denen die jüngere schon in der Kindheit zu 
Schlafstörungen neigt und sich in den späten dreißiger Jahren in Behandlung begibt. Dieser Patiententyp ringt verzweifelter um seinen Schlaf, ist ängstlicher und grüblerischer als die andere, erst im Alter von Anfang fünfzig therapeutische Hilfe suchende Gruppe (Edinger et al. 1988). Mindestens drei Patientengruppen ließen sich mittels Cluster-Analyse des FPI voneinander abgrenzen: Während Gruppe 1 sich in ihrem Selbstbild nicht von Gesunden unterschied, zeigte das Persönlichkeitsprofil von Gruppe 2 erhöhte emotionale Labilität und Erregbarkeit, eine Tendenz zu psychosomatischen Beschwerden und herabgesetzte Lebenszufriedenheit; Gruppe 3 der Insomniepatienten war introvertierter sowie gehemmter und unsicher im sozialen Kontakt; Leistungsorientierung, Aggressivität und Reaktivität waren herabgesetzt, trotzdem fühlten sich die Patienten stark beansprucht und überfordert (HerrmannMaurer et al. 1988).

\subsubsection{Symptome}

Schlafgestörte Patienten leiden während der Nacht an unterschiedlichen Symptomen: Der Schlaf kann zu kurz oder zu unruhig sein oder als zu wenig erholsam empfunden werden, bei einer Einschlafstörung kann er sich erst nach längerem Wachliegen einstellen. Er kann durch häufiges nächtliches Erwachen unterbrochen werden und mit langem Wachliegen verbunden sein (vgl. Hajak und Rüther 1995). Auch das Tagesbefinden ist deutlich beeinträchtigt. Die Patienten sind besorgt, aufgrund des Schlafmangels den Anforderungen des Tages nicht gewachsen zu sein. Sie sind deprimiert, angespannt und vorrangig mit sich selbst beschäftigt (Kales und Kales 1984). Die täglichen Verrichtungen fallen dem Schlafgestörten schwerer, es fehlt an Kraftreserven und an Spontanität (Hauri 1979). Tatsächlich jedoch ist die Leistungsfähigkeit während des Tages nicht eingeschränkt, zumindest lässt sie sich für die Testdauer mobilisieren. Nur bei längerdauernden, monotonen Aufgaben ist die Aufmerksamkeit gemindert.

Ermüdung am Tage ist bei Insomniepatienten sehr verbreitet (Mendelson 1993, Lichstein et al. 1997), die Neigung, während des Tages einzuschlafen, nimmt bei ihnen jedoch mit der steigenden Zahl nächtlicher Aufwachvorgänge ab. Dies steht im Gegensatz zur reaktiven Hypersomnie bei Gesunden (Stepanski et al. 1984). Andere Autoren grenzen einen weiteren Insomnikertyp ab, der weniger an Ermüdung leidet als vielmehr an Angst, vager Dysphorie, Nervosität, dem Gefühl, „ein Band um den Kopf“ zu haben, Gefühlen der Depression, Beeinträchtigung, Reizbarkeit und dergleichen mehr (Dement et al. 1984). 
Eine multizentrische Studie zeigte auf, dass die gesundheitsbezogene Lebensqualität von Insomnikern deutlich gemindert ist und Gesundheitseinrichtungen von ihnen vermehrt genutzt werden (Hatoum et al. 1998). Aufgrund verstärkter nächtlicher Aktivität des sympathischen Nervensystems könnten Insomniker anfälliger für die Entwicklung einer koronaren Herz- und anderer Krankheiten sein (Bonnet und Arand 1998).

Ein weiteres Charakteristikum ist die Sorge selbst, nicht ein- oder durchschlafen zu können, doch steigende Anspannung und Erregung lassen alle dahingehenden Bemühungen scheitern, was wiederum zu stärkerer Besorgnis um ausreichend Schlaf und Leistungsfähigkeit am Tage führt. Der Schlaf stellt sich oft gerade dann ein, wenn er nicht gewollt ist, wie zum Beispiel vor dem Fernseher. Eine ungewohnte Umgebung kann ebenfalls den Schlaf verbessern (APA 1987). Es resultiert das Gefühl, der Schlaflosigkeit machtlos gegenüberzustehen, sie nicht selbst in den Griff bekommen zu können (Lacks und Morin 1992).

Schlafstörungen haben einen ungünstigen Einfluss auf das soziale Leben, die persönlichen Beziehungen und die berufliche Situation der Betroffenen (Kales et al. 1984). Letztere wird bei einem Viertel der Insomniepatienten beeinträchtigt durch Müdigkeit, Depression, Nervosität, Angst oder den Gebrauch von Drogen und Alkohol. Schlafgestörte sind für den Gebrauch dieser Substanzen prädisponiert (Weissman et al. 1997, Gillin 1998), die ihrerseits den Schlaf negativ beeinflussen können (ASDA 2001).

\subsubsection{Wahrnehmung und Fehlwahrnehmung des Schlafes}

Neurotische und besorgte Patienten leiden verstärkt unter den Nachwirkungen des Schlafmangels (Coyle und Watts 1991), der gerade bei diesen Patienten oft nicht polysomnografisch nachgewiesen werden kann. Einige Autoren vermuten, Patienten mit einer Charakterstörung oder der Unfähigkeit zu zwischenmenschlichen Beziehungen (Dement et al. 1984) zögen einen Krankheitsgewinn aus der Schlafstörung, die es ihnen ermöglicht, sich einem Konflikt zu entziehen (Trilling 1992). Als weitere Ursachen für eine Diskrepanz zwischen wahrgenommener und nachgewiesener Schlafstörung vermuten Dement et al. (1984) ein gesteigertes Schlafbedürfnis oder das Vorhandensein einer mit der Standard-Schlafauswertung (Rechtschaffen und Kales 1968) nicht nachweisbaren Veränderung des Schlafes. Zwei mögliche Ursachen für die Fehlwahrnehmung des Schlafes wären möglich: entweder ein Übergangsstadium zur polysomnografisch manifestierten Insomnie oder aber eine dazu prädisponierte Persönlichkeit, da diese Patienten im Gegensatz zu anderen Insomnikern ein vor allem 
auf der Hysterieskala verändertes MMPI-Profil aufwiesen (Salin-Pasqual et al. 1992). Für das Übergangsstadium spricht eine nachgewiesene Erhöhung der Stoffwechselrate (Bonnet und Arand 1997).

Auch Insomniepatienten mit positiven Befunden in der Polysomnografie schätzen ihren Schlaf schlechter ein, als er tatsächlich ist: Im Gegensatz zu guten Schläfern neigen sie verstärkt dazu, Einschlafzeiten und nächtliche Wachzeiten zu über- und ihre Gesamtschlafdauer zu unterschätzen (Dement et al. 1984). Werden sie kurz nach dem Einschlafen geweckt, so sind sie sich öfter als gute Schläfer nicht bewusst, schon geschlafen zu haben (Coates et al. 1983, Mendelson et al. 1986, Rechtschaffen 1968). Neuere Mikrostrukturanalysen haben gezeigt, dass sich in der Einschlafperiode der Schlaf von Insomniepatienten bezüglich der Alphaaktivität unterscheidet und hier ein möglicher Grund für die Fehlwahrnehmung des Schlafes liegen könnte (Lamarche und Ogilvie 1997).

Die Nacht-zu-Nacht-Variabilität kann bereits bei gesunden Menschen dadurch zustande kommen, dass durch Stress, der durch die Erwartung des nächsten Arbeitstages ausgelöst wurde, der Tiefschlaf reduziert sein kann (Kecklund und Akerstedt 2004); durch emotionale Anspannung kann hauptsächlich der erste Schlafzyklus strukturell verändert werden, indem der Deltawellenschlaf vermehrt in die zweite Nachthälfte verlagert wird; des Weiteren nimmt die Frequenz und Variabilität des Herzschlags im Schlaf zu (Vein et al. 2002). Nacht-zu-Nacht-Variabilität kann bei schlechten Schläfern sehr ausgeprägt sein (Hauri 1989), sie ist im Schlaflabor bewiesen (Coates et al. 1982) und wird in ihren negativen Auswirkungen auf die Tagesbefindlichkeit als besonders belastend empfunden.

\subsubsection{Diagnostik der Insomnie}

Zur Diagnostik der Insomnie steht zur Erfassung der subjektiven Schlafparameter eine Vielzahl von Fragebögen zurVerfügung. Die Polysomnografie gibt Aufschluss über die Makrostruktur und das Ausmaß der Schlaffragmentierung, letzteres mittels der Erfassung von Mikro-Arousals oder weniger zeitintensiver Verfahren wie des SchlafFragmentations-Indexes (Haba-Rubio, Ibanez und Sforza 2004) und der HandgelenksSimultanmessung von peripherem arteriellen Tonus, Oximetrie und Aktigrafie (Pillar et al. 2003). Die Polysomnografie erlaubt zudem den Ausschluss anderer Schlafstörungen wie des Restless-Legs-Syndroms und schlafbezogener Atemstörungen. Die Aktigrafie ist der Polysomnografie in der Einfachheit der Anwendung überlegen, steht im Informationsgehalt hinsichtlich einzelner Schlafparameter jedoch deutlich zurück 
(Ancoli-Israel et al. 2003). Neuere Verfahren zur computergestützten Auswertung nach den immer noch einzigen weltweit anerkannten Standart-Kriterien zur Schlaf-EEGAuswertung von Rechtschaffen und Kales 1968 wie der Somnolyzer 24x7 erscheinen vielversprechend, da hier eine 80\%ige Zuverlässigkeit nachgewiesen wurde, die mit einer Inter-Rater-Übereinstimmung von 77\% durchaus mithalten kann (Anderer et al. 2005). Polysomnografische Ableitungen wurden mittels eines automatischen SleepStaging-Systems und eines EEG-abgeleiteten Spektralfrequenzindexes (SFx) ausgewertet und die SFx-Werte wurden mit Daten von SF-A-Fragebögen in Beziehung gesetzt. Der Vorteil dieser Methode ist gegenüber der visuellen Schlafauswertung, dass hierbei die Tiefe des Schlafes objektiv festgehalten wird (Hammer et al. 2001). Mittels neuer Auswertungskriterien im 4-Sekunden Rhythmus mögen Instabilitäten bezüglich des Stadienwechsels in der initialen Einschlafphase besser zu erfassen sein (Moul et al. 2007). Verschiedene Methoden zur Erfassung von Schlafspindeln sind unterschiedlich genau (Huupponen et al. 2007).

\subsubsection{Behandlung der Insomnie}

Behandelt werden sollte eine Insomnie dann, wenn die diagnostischen Kriterien dafür (s. Kapitel 1.1.1.) erfüllt sind.

Vor der Empfehlung einer Behandlung sind patientenspezifische Kriterien wie Alter, Krankengeschichte, Vormedikation und die der Schlafstörung zugrunde liegende Ursache zu berücksichtigen (Morin, Jarvis und Lynch 2007). Die Therapieformen lassen sich in medikamentöse und nichtmedikamentöse Behandlungsformen einteilen, die wesentlichen sind im Folgenden in Grundzügen dargestellt. Bei anhaltender Insomnie über drei Monate trotz durchgeführter Behandlung sollte ein Spezialist für Schlafmedizin konsultiert werden (Clarenbach et al. 1995).

\subsubsection{Medikamentöse Therapieformen}

Pharmakotherapie ist bislang die vorherrschende Behandlungsform der Insomnie (Sievertsen und Nordhus 2007), ihre Anwendung ist seit 1987 jedoch um ein Viertel zurückgegangen (Walsh und Schweitzer 1999). Die Hälfte der Patienten beklagt auch unter Medikation anhaltende Beschwerden (Hohagen et al. 1993, Holzrichter et al. 1994, Simen et al. 1995).

Am häufigsten wurden bis vor zehn Jahren Benzodiazepine verschrieben (Morin et al. 1994). Diese senken kurzfristig die Einschlaflatenz und die Anzahl und Dauer nächtlicher Aufweckvorgänge, sie steigern die Gesamtschlafdauer und die 
Schlafeffizienz (Gillin und Byerley 1990, Morin und Kwentus 1988) und können im letzten Drittel der Nacht den Schlaf aufrechterhalten (Erman, Loewy und Scharf 2005). Die Behandlung mit Benzodiazepinen hat jedoch eine Reihe gravierender Nachteile und birgt die Gefahr von Missbrauch und Abhängigkeit (Hajak et al. 1997) sowie insbesondere beim älteren Menschen die Gefahr der Überdosierung und Kumulation. Bei kurzwirksamen Benzodiazepinen setzt schon nach kurzer Zeit eine Gewöhnung ein, die Wirkung ist bereits vor Ablauf der Nacht beendet, und nach Absetzen ist eine Rebound-Insomnie besonders stark ausgeprägt; längerwirksame Substanzen dagegen verursachen durch ihre über die Nacht hinaus anhaltenden Wirkung oft morgendliche Müdigkeit, auch sie verlieren bei langfristigem Gebrauch an Effekt, der Schlaf verschlechtert sich im Vergleich zum Therapiebeginn sogar (Gillin und Byerley 1990, Morin und Kwentus 1988).

In den letzten zwei Jahrzehnten ist es zu einem deutlichen Rückgang der Benzodiazepine um über 50\% zugunsten von Antidepressiva gekommen, über deren Wirksamkeit und Nebenwirkungen nur wenige Daten vorliegen (Walsh und Schweitzer 1999). Niedrig dosiert kann das trizyklische Antidepressivum Doxepin die Schlafeffizienz und die Arbeitsfähigkeit von Patienten mit chronischer primärer Insomnie verbessern, es kam jedoch zu signifikant häufigerer Rebound-Insomnie als in der Plazebo-Gruppe, zum Transaminasen-Anstieg und zu Blutbildveränderungen (Hajak et al. 2001). Die Anwendung von Neuroleptika ist durch unerwünschte Wirkungen eingeschränkt (Hajak und Rüther 1993). Andere Nichtbenzodiazepine können nebenwirkungsarme Alternativen sein, z.B. wird das Imidazopyridin Zolpidem von Hausärzten durchweg als effektives Hypnotikum beurteilt. Es konnte damit eine signifikante Verbesserung der objektiven Schlafqualität erreicht werden, die subjektiven Veränderungen gingen jedoch nicht über den Plazeboeffekt hinaus (Saletu-Zyhlarz et al. 2000). Eine Rebound-Insomnie und eine Toleranzentwicklung zeigte sich in der Untersuchung von Monti et al. 1994 bei diesem Präparat im Gegensatz zum Benzodiazepin Triazolam nicht, es konnten sich hier sowohl objektive als auch subjektive Schlafparameter bessern. Eine kleine mexikanische Studie konnte eine Reduktion nächtlicher Wachperioden durch Valeriana edulis ssp. procera, besser bekannt als Valeriana mexicana, und V. officinalis sowie eine REM-Schlaf-Zunahme, v.a. durch die letztere Substanz polysomnografisch nachweisen (Herrera-Arellano et al. 2001). Die begrenzte Dauer von Medikamentenstudien sowie der Mangel an Katamnesen (Nowell et al. 1997b) geben jedoch wenig Aufschluss über die Anwendung bei chronischer Insomnie. 
Allen Medikamenten gemeinsam ist eine nachteilige psychologische Wirkung: Der Patient führt die Verbesserung des Schlafes allein auf das Medikament zurück und nicht auf Faktoren, die er selbst beeinflussen kann. Dies bestärkt ihn in seiner Überzeugung, der Schlaflosigkeit machtlos gegenüberzustehen, sie nicht selbst in den Griff zu bekommen. Diese passive Haltung macht ihn für eine anschließende nichtmedikamentöse Therapie wenig empfänglich. Studien belegen, dass medikationsfreie Patienten von einer nichtmedikamentösen Therapie mehr profitieren als zuvor medikamentös behandelte Patienten, die die gleiche Therapie und ein Entzugsprogramm erhielten (Riedel et al. 1998, Murtagh und Greenwood 1995) und langfristig auch gegenüber Patienten, die gelegentlich Hypnotika konsumieren. Dies liegt zum Teil daran, dass die kausale Therapie der Insomnie vernachlässigt wird, weil durch prompte Beschwerdebesserung nach Beginn der Medikamenteneinnahme der Leidensdruck zunächst gemindert wird. Zudem kann eine der Schlafstörung möglicherweise zugrundeliegende Erkrankung wie beispielsweise eine Angststörung oder Depression durch das Medikament verschleiert werden. Nach herrschender Meinung sollte jede medikamentöse Therapie nicht isoliert durchgeführt, sondern mit einer nichtmedikamentösen Behandlung kombiniert werden (Hajak et al. 1997). Die Patienten selbst bevorzugen, wenn sie wählen können, nichtmedikamentöse Therapieformen (Stepanski 2005).

Eine Sonderform medikamentöser Behandlungen ist die Hormonsubstitution, deren Einfluss auf den Schlaf bislang wenig untersucht worden ist. In den USA verbreitet ist der Gerbrauch von Melatonin, das zumindest auf den Schlaf älterer Menschen positiven Einfluss hat (Brusco et al. 1999), den REM-Schlaf-Anteil erhöhen kann, dessen Bedeutung für Gehirnfunktionen, metabolische Prozesse und das Immunsystem erst im letzten Jahrzehnt zunehmend erkannt wurde (Kunz et al. 2004). Bei der von Insomnie besonders betroffenen Gruppe der postmenopausalen Frauen kann Östrogensubstitution unter Minderung der klimakterischen Symptome den subjektiven Schlaf und die Tagesmüdigkeit verbessern (Polo-Kantola et al. 1998).

\subsubsection{Nichtmedikamentöse Therapieformen}

Seit langem fordern Schlafforscher, den Schwerpunkt auf nichtmedikamentöse Behandlung zu setzen (Hajak et al. 1997), denn chronische Insomnien entstehen zumeist aus einer Interaktion von physiologischen, kognitiven, psychosozialen und behavioralen Faktoren (Espie 1991). Mit nichtmedikamentösen Therapieformen kann daher nicht nur symptomatisch, sondern auch kausal der Schlafstörung begegnet werden, auch geben Insomniepatienten selbst diesen den Vorzug gegenüber medikamentöser Behandlung 
(Morin et al. 1992). Einige Autoren empfehlen die Kombination beider Therapieformen (Vgontzas und Kales 1999), andere wiesen die Überlegenheit der alleinigen Durchführung einer nichtmedikamentösen Therapie gegenüber der Kombination mit Pharmakotherapie nach (Hauri 1997). Langfristig gesehen wird sich nichtmedikamentöse Behandlung im Vergleich zur Pharmakotherapie möglicherweise als kostengünstiger erweisen (Morin et al. 1994).

\subsection{Grundlegende Maßnahmen: Aufklärung, Beratung und Schlafhygiene} Informationsvermittlung über den Schlaf und seine Variationsbreite sowie Ursachen und Konsequenzen seiner Störung sind die Voraussetzung für jede Behandlung von Schlafstörungen. Insomniepatienten haben bezüglich ihres Schlafes oft falsche Erwartungen und Vorstellungen (Morin et al. 1993), die ihrerseits das Schlafproblem aufrechterhalten können. Durch Aufklärung können falsche Attributionen korrigiert und Missverständnisse über den Schlaf und seine Störung aufgeklärt werden (Espie 1991).

Schlafhygiene ist Bestandteil der meisten kognitiven und Verhaltenstherapien, allein angewendet ist sie jedoch nicht ausreichend (Schoicket et al. 1988, Morgenthaler et al. 2006). Sie klärt den Patienten über den Schlaf und seine Störung auf und leitet dazu an, den Schlaf verhindernde Verhaltensweisen abzustellen und eine schlaffördernde Umgebung zu schaffen. Eine genaue Darstellung der Inhalte erfolgt in Kapitel 1.2.3.

Schlechter Schlaf kann durch mangelnde Schlafhygiene verursacht sein, er kann sie jedoch auch bedingen (Nau und Walsh 1983). Bei einer Studie trat zutage, dass Patienten mit Einschlafproblemen über bessere Kenntnisse der Schlafhygiene verfügen als gute Schläfer, jedoch davon weniger Gebrauch machen. Auch sind sie sich des Zusammenhangs von Verhalten und Schlaf bewusster als gute Schläfer, sie verhalten sich jedoch weniger schlaffördernd. Ältere Patienten praktizieren Schlafhygiene am stärksten. Da das Wissen über Schlafhygiene und die Anwendung derselben sowohl bei guten als auch bei schlechten Schläfern generell hoch ist, scheint ihr Mangel in den meisten Fällen nicht die Hauptursache für gestörten Schlaf zu sein (Lacks und Rotert 1986). In einer Studie über praktizierte Schlafhygiene bei Frauen zeigte sich, dass Frauen, die an Insomnie litten, weniger Kaffee tranken, häufiger Alkohol-abstinent waren und eine geringere Variationsbreite im täglichen Alkoholkonsum zeigten als gute Schläferinnen (Cheek, Shaver und Lentz 2004). 


\subsection{Entspannungsverfahren}

Zu den Entspannungsverfahren zählen Biofeedback, autogenes Training nach Schultz, Meditation und die progressive Muskelrelaxation nach Jacobson.

Musik des Gehirns wurde von russischen Forschern entwickelt, indem sie EEG in Musik transformierten. Die Patienten hörten ihr eigenes EEG vor dem Einschlafen an, die Kontrollgruppe lauschte fremden EEGs. Der Autor berichtet von subjektiver und objektiver Wirkung in 80\% bei fehlenden Nebenwirkungen (Levin 1998).

Eine weitere Möglichkeit des Biofeedbacks ist die Rückmeldung der eigenen Muskelanspannung mittels akustischer Signale (Hauri 1981, Nicassio et al. 1982). Patienten, die für Entspannungsverfahren nicht zugänglich sind, lernen auf einem indirekten Weg den Spannungs- und Entspannungszustand ihrer Muskulatur wahrzunehmen. Wegen des Erfolges von als Placebo dienenden Pseudo-BiofeedbackTherapien vermuten Morin et al. (1994), dass die Wahrnehmung der Kontrolle in höherem Maße für die Schlafverbesserung verantwortlich ist als die Kontrolle selbst.

Yoga, bislang zur Insomniebehandlung noch wenig untersucht, konnte in der täglichen Anwendung bei einer kleineren Stichprobe in der subjektiven täglichen Beurteilung statistisch signifikant die Schlafparameter Schlafeffizienz, Gesamtschlafzeit, Gesamtwachzeit, nächtliche Wachzeit und Einschlaflatenz verbessern (Khalsa 2005).

Beim Autogenen Training wird ein Zustand tiefer Entspannung durch die Suggestion bestimmter, auf einzelne Körperregionen bezogener Empfindungen erreicht. Hierzu denkt der Patient sich in entspannter Körperhaltung einer Reihe von Standardsätzen (Schultz und Luthe 1959) folgend in seinen Körper hinein. Voraussetzung für das erfolgreiche Nachempfinden von Wärme, Schwere etc. ist regelmäßiges, gar tägliches Üben (Eberlein 1996). Eine Minderung der Einschlaflatenz sowie eine globale Verbesserung wurden von Nicassio und Bootzin (1974) beobachtet, letztere verlor sich jedoch nach Therapieende.

Anspannungs-Entspannungsübungen wie die progressive Muskelrelaxation bewirken neben physischer Entspannung die Verringerung einschießender Gedanken beim Einschlafen, auch die Anspannung am Tag kann verringert werden (Borkovec und Hennings 1978). Sie wirken vermutlich durch kognitive Entspannung (Espie 1991). Die Entspannungsverfahren sind hinsichtlich der Einschlafdauer, weniger jedoch bei Durchschlafproblemen praktisch immer erfolgreich und sind einfach zu erlernen. Bis 
zum Auftreten deutlicher Erfolge muss allerdings mit 6 Wochen gerechnet werden, die Motivation spielt dabei eine wichtige Rolle (Friebel und Friedrich 1992).

Die Progressive Muskelrelaxation nach Jacobson lässt den Patienten die zwei gegensätzlichen Empfindungen Anspannung und Entspannung durch Muskelübungen erfahren und erzielt ein Gefühl tiefer Entspannung (Pritz 1990). Diesem Verfahren, das sich bereits zur Therapie von Insomnien bewährt hat, ist als Gegenstand dieser Untersuchung das Kapitel 1.3 gewidmet. Borkovec et al. $(1975,1979)$ sahen sowohl die gerichtete Aufmerksamkeit auf Spannungszustand und -änderung der Muskulatur als auch den Vorgang von An- und Entspannung selbst als die beiden wesentlichen Komponenten der progressiven Relaxation an. Andere Autoren konnten die wesentliche Bedeutung des letzteren Elementes jedoch nicht bestätigen (Woolfolk und McNulty 1983, Haynes et al. 1974, Nicassio und Bootzin 1974). Auch Espie (1991) konnte keinen signifikanten Zusammenhang zwischen dem Grad der physiologischen Erregung und der Einschlaflatenz feststellen und befand, dass Muskelentspannung allein nicht für die verkürzte Einschlaflatenz verantwortlich zu sein scheint. Die meisten Patienten geben Grübeln als Ursache ihrer Einschlafstörung an (Freedman und Papsdorf 1976). Dieses abzustellen könnte dem Einschlafen gar förderlicher sein als die Verminderung körperlicher Anspannung (Woolfolk und McNulty 1983) und kann mit einem kognitiven Therapieansatz erreicht werden.

\subsection{Verhaltenstherapeutische, kognitive und regulierende Techniken}

Verhaltenstherapeutische und kognitive Techniken sind nichtmedikamentöse Behandlungsformen, die auf unterschiedliche Weise maladaptive Verhaltensweisen ändern und das Einschlafen verhindernde Gedanken fernhalten sollen.

Paradoxe Intention durchbricht den Teufelskreis, der entsteht, wenn der Patient versucht, willentlich den Schlaf herbeizuführen. Der Selbstbeobachtung - ich liege wach und kann nicht einschlafen - folgt zunehmende Erregung über das eigene Unvermögen sowie die Angst, nicht einschlafen zu können und am nächsten Tag nicht ausgeruht zu sein. Daraus resultierende verstärkte Bemühungen einzuschlafen scheitern zwangsläufig, da der Eintritt des Schlafes ein gänzlich unwillkürlicher physiologischer Prozess ist (Espie 1991). Erst die paradoxe Absicht, wach zu bleiben anstatt einzuschlafen, erzielt den gewünschten Effekt.

Stimuluskontrolle zielt darauf ab, durch die von Bett und Schlafzimmer ausgehenden Reize den Schlaf herbeizuführen. Daher müssen andere Verrichtungen als Schlaf und Sex sowie das Wachsein räumlich ferngehalten werden, das heißt bei Schlaflosigkeit 
wird das Zimmer verlassen und erst nach entspannender Tätigkeit bei erneut auftretender Müdigkeit wieder aufgesucht. Regelmäßige Weckzeiten und Verzicht auf Schlaf während des Tages sind weitere Maßnahmen zur Stimuluskontrolle, die sich sowohl bei jüngeren als auch bei älteren Patienten als wirksam herausgestellt hat. In den meisten Studien wurde vor allem eine baldige Reduktion der Einschlaflatenz erreicht. (Espie 1991)

Schlafrestriktion setzt an der geringen Schlafeffizienz an (Schlafzeit in Prozent der Gesamtzeit im Bett), die daher rührt, dass Insomniepatienten viel Zeit im Bett mit Wachliegen verbringen (Spielman et al. 1987). Zu Beginn der Therapie wird eine feste Weckzeit eingerichtet, an der sich die Zubettgehzeit orientiert, die zunächst spät gewählt wird. Es wird dem Patienten nur die Zeit im Bett zugebilligt, die er als seine übliche Gesamtschlafzeit angibt. Das heisst, wenn er angibt, durchschnittlich 5 Stunden zu schlafen, dann darf er zu Beginn der Therapie auch nur diese Zeit im Bett verbringen. Durch diese anfänglich starke Zeitbegrenzung soll durch Erhöhung des Schlafdruckes eine hohe Schlafeffizienz erreichet werden, im Therapieverlauf kann bei anhaltend guter Schlafeffizienz die Bettzeit ausgedehnt werden. Friedman et al. (1991) untersuchten diese Behandlungsform im Vergleich mit Entspannungstherapie bei älteren Patienten. Einschlaflatenz und nächtliche Wachzeit reduzierten sich bei beiden Patientengruppen, wobei im Drei-Monats-Follow-up die verbesserte Schlafeffizienz nur bei der mit der Schlafrestriktion behandelten Gruppe beobachtet werden konnte.

Sleep Retraining ist eine neuartige Kurzzeittherapie zur Behandlung der chronischen primären Insomnie. Sie beinhaltet eine einzelne Nacht des Schlafentzugs mit nachfolgenden Gelegenheiten zu einem kurzen Nickerchen. Hiermit ließ sich bei einer kleineren Stichprobe der Schlaf verbessern, die Verbesserungen hielten zwei Monate über das Behandlungswochenende hinaus an. (Harris et al. 2007)

Ein dem „Schafezählen“ ähnliches Gedankenstop-Verfahren blockiert wiederholte, aber nicht gefühlsbeladene Gedanken (Cautela und Wisocki 1977), kann aber bei stark belastenden Gedankeninhalten versagen.

Imaginationstraining bzw. kognitive Fokussierung lenken von negativen und ungerichteten sowie sich schnell ändernden Gedanken ab, indem die Aufmerksamkeit auf eine vorgestellte ruhige Situation oder ein vorgestelltes Objekt gelenkt wird. Diese Behandlung konnte als Einzeltherapie nicht empfohlen werden aufgrund unzureichender Evidenz (Morgenthaler et al. 2006). 
Über kognitive Kontrolle liegen bisher nur wenige Publikationen vor. Sie wirkt präventiv und zielt darauf ab, dem das Einschlafen verhindernden Grübeln vorzubeugen, indem sie für Planungs- und Bilanz ziehende Gedanken eine feste Zeit am früheren Abend bereitstellt (Espie und Lindsay 1987). Durch die regelmäßige Auseinandersetzung mit Ereignissen des Tages sowie anliegenden Problemen am frühen Abend gibt es nachts nichts mehr zum Grübeln.

Kognitive Therapie, bei der negative Gedanken bewusst gemacht werden, auf ihren Realitätsgehalt hin überprüft werden sowie schließlich durch realistische angemessene Gedanken ersetzt werden, konnte als Einzeltherapie nicht empfohlen werden aufgrund unzureichender Evidenz (Morgenthaler et al. 2006).

Auch zur Akupunktur gibt es nur wenig Datenmaterial, bei einer kleinen Stichprobe hat sie sich als wirksam erwiesen (Montakab 1999).

Selbsthilfegruppen sind eine weitere, kostengünstige Möglichkeit, die bisher noch nicht evaluiert wurde. Aufgrund der geringen gesellschaftlichen Akzeptanz der Insomnie als behandlungswürdige Störung fühlten sich darunter leidende Patienten bisher oft alleingelassen. Das Gefühl der Machtlosigkeit, der Schlaflosigkeit allein ausgeliefert zu sein, könnte gemildert werden, wenn der Patient sich dessen bewusst ist, dass er zumindest Leidensgenossen hat, was ihm angesichts des zumeist hervorragenden Schlafes des Bettpartners nicht bewusst wird. Zur Umsetzung einer schlaffördernden Lebensweise gibt die Gruppe Motivation, und im Sinne von Weight Watchers könnte ein Gruppendruck die Umsetzung schlaffördernden Verhaltens bewirken, an der es Insomniepatienten oft mangelt (Lacks und Rotert 1986).

\subsection{Psychotherapie im engeren Sinne}

In der Verhaltenstherapie werden zunächst die Faktoren identifiziert, die die Schlafstörung verursachten und aufrechterhalten. Sie zielt darauf ab, die Funktionalität der Störung zu bearbeiten, das gelernte maladaptive Verhalten $\mathrm{zu}$ ändern und dem Patient Bewältigungsstrategien zu vermitteln, dies können unter anderem die bereits aufgeführten Techniken sein (s. Kapitel 1.1.7.2.3).

Kognitiv-behaviorale Therapie reduziert dysfunktionale Meinungen über den Schlaf, was mit anderen positiven Ergebnissen in der Insomniebehandlung assoziiert ist (Edinger et al. 2001). Nach der Einsicht in die Dysfunktionalität bestimmter Denkmuster werden alternative funktionale Denkmuster erarbeitet und praktisch eingeübt (Beck 1995). Verhaltensänderung und die Veränderung von Denkmustern 
beeinflussen sich gegenseitig. Kognitiv-behaviorale Therapie ist zur Behandlung der chronischen Insomnie der medikamentösen Behandlung im Langzeit-Management der Insomnie überlegen (Stepanski 2005). Die Methode kann auch in Kleingruppen durch trainierte und supervidierte Gemeindeschwestern durchgeführt werden, wenn auch mit eingeschränkter Effektivität; ob sie in der medizinischen Grundversorgung Therapie erster Wahl für persistierende Insomnie werden kann, müsste weiter erforscht werden (Espie et al. 2007).

Über analytisch orientierte Therapie zur Behandlung der Insomnie liegen kaum Publikationen vor (Hoffmann 1980, Hajak et al. 1997).

Interpersonelle Psychotherapie für Insomnie betrachtet den psychosozialen Kontext, in dem sich der Patient mit seiner Schlafstörung befindet. Wie die analytische Therapie arbeitet sie ursachenorientiert, im Gegensatz zu ihr jedoch gegenwartsbezogen. Ein oder zwei zwischenmenschliche Problembereiche, die mit der Auslösung beziehungsweise Aufrechterhaltung der Schlafstörung in Zusammenhang stehen, werden bearbeitet, des weiteren wird der Tagesablauf auf mögliche schlafstörende Verhaltensweisen (Hajak et al. 1997) untersucht. Dieses Verfahren ist Gegenstand der vorliegenden Untersuchung, es wird in Kapitel 1.2.3 ausführlich dargestellt.

\subsection{Behandlungsdauer}

Die Dauer der Behandlung spielt im Gesundheitswesen aufgrund der Kosten eine immer größere Rolle. Die Literatur zu Behandlungszeiträumen ist spärlich, über analytisch orientierte Therapien forschten Kordy, von Rad und Senf 1989 und kamen ungeachtet der Diagnosen zu einem besonders effektiven Behandlungsmodell von 160 Sitzungen innerhalb von 2,5 Jahren. Um bei Krankheiten mit einem hohen Rückfallrisiko wie der rezidivierenden Depression das beschwerdefreie Intervall zu verlängern, wurden nach Beendigung der Therapie die Interpersonelle Psychotherapie als Erhaltungstherapien mit monatlichen Sitzungen fortgeführt, mit Erfolg (Frank et al. 1991). 


\subsection{Vergleich}

Morin et al. verglichen 1994 in einer Meta-Analyse die Wirksamkeit von 59 Studien über nichtmedikamentöse Therapien der Insomnie. Untersucht wurden: somatische und kognitive Entspannungstherapie, Biofeedback, paradoxe Intention, Erziehung in Schlafhygiene, Multikomponententherapie, Stimuluskontrolle und Schlafrestriktion. Die beiden letzteren stellten sich dabei als die effektivsten aller Einzeltherapien heraus, Schlafhygiene erwies sich bei alleiniger Anwendung als nicht ausreichend. Im Vergleich von Einzel- zu Gruppen- und Selbstanwendungstherapien nahm die Effektivität in dieser Reihenfolge ab. Patienten mit Einschlafstörungen verbesserten sich stärker als jene mit Durchschlafstörungen. Murtagh und Greenwood (1995) konnten in ihrer MetaAnalyse von 66 Studien über psychologische Behandlungsformen deren Überlegenheit gegenüber Placebo, jedoch keinen wesentlichen Unterschied der verschiedenen Therapien in ihrer Wirksamkeit feststellen, allenfalls eine leichte Überlegenheit der Stimuluskontrolle. Überwiesene Patienten und solche ohne regelmäßige Schlafmedikation profitierten stärker als andere.

Grundsätzlich ist ein Vergleich der verschiedenen Therapieformen schwierig, da neben den therapieeigenen Effekten unterschiedliche Faktoren den Behandlungserfolg beeinflussen: Art (Ein-/ Durchschlafstörung), Schwere und Chronifizierungsgrad der Erkrankung, Motivation, Alter, Geschlecht und Bildungsstand des Patienten, Qualifikation der Therapeuten, Art der Erfolgskontrolle, Rahmenbedingungen sowie weitere unspezifische Faktoren.

Die einzelnen Behandlungsarten wirken sich unterschiedlich auf den Schlaf aus: Stimuluskontrolle ändert quantitativ das Schlafmuster, scheint jedoch auf die Schlafqualität einen geringeren Einfluss zu haben. Die Entspannungsverfahren stellen sich dagegen quantitativ bezüglich Einschlaflatenz und nächtlicher Wachzeit weniger erfolgreich dar, wirken sich jedoch auf die Befindlichkeit am Tage positiv aus, indem sie den Patient befähigen, Angst und Anspannung nicht nur während der Nacht zu reduzieren. Die Hypothese, Entspannungsverfahren könnten langfristig auch bezüglich der subjektiven Schlafqualität überlegen sein (Espie et al. 1989), fand sich nicht bestätigt (Murtagh und Greenwood 1995). Ein bedeutsamer Faktor für den Therapieerfolg ist die Compliance (Chambers 1992). Sie ist bei einigen Therapien wie z.B. der Stimuluskontrolle der den Behandlungserfolg limitierende Faktor (Hajak et al. 1997). Wichtig ist, dass Arzt und Patient die gleichen Vorstellungen über das Therapieziel haben (Espie 1991). Die Art der Beeinträchtigung durch die Schlafstörung kann für den Erfolg einer ganz bestimmten Behandlung sprechen: Lichstein et al. 2001 
fanden bei der Untersuchung an älteren Insomniepatienten heraus, dass Patienten mit einer hohen Beeinträchtigung der Befindlichkeit am Tage am besten von einer Behandlung profitieren, die zu einer Verlängerung des Schlafes führt, wie der Relaxation, und dass die älteren Insomniepatienten, die nur eine geringe Beeinträchtigung der Tagesbefindlichkeit aufweisen, eher von einer Konsolidierung des Schlafes, wie sie durch Schlafkompression erreicht werden kann, profitieren.

Morin et al. (1994) kommen zu dem Schluss, dass mit nichtpharmakologischen Methoden insgesamt verlässliche und dauerhafte, wenn auch nur moderate Therapieerfolge erzielt wurden. Von einer durch die American Academy of Sleep Medicine gebildeten Projektgruppe wurde eine Metaanalyse von 37 zwischen 1998 und 2004 veröffentlichten Studien durchgeführt, in denen Patienten mit Insomnie als Hauptund Nebendiagnose mit wenigstens einem psychologischen oder verhaltenstherapeutischen Therapieverfahren behandelt worden waren. Mindestens eine der abhängigen Variablen Einschlaflatenz, Anzahl oder Dauer der nächtlichen Wachphasen, Gesamtschlafzeit, Schlafeffizienz oder Schlafqualität war untersucht worden. Hierbei zeigte sich, dass es zu zuverlässigen Veränderungen mehrerer dieser Schlafparameter kam. Es gab jedoch nur begrenzt Evidenz bezüglich des Einflusses auf andere Morbiditäts-Indices wie z. B. Tagesmüdigkeit. Fünf der bereits im Kapitel 1.1.7.2 näher erläuterten Behandlungsverfahren erfüllten die Kriterien für empirische Wirksamkeit: Stimuluskontrolle, Relaxation, Paradoxe Intention, Schlafrestriktion und kognitiv-behaviorale Therapie (Morin et al. 2006). Morgenthaler et al (2006) stellten diese Ergebnisse aufgeschlüsselt nach den differenzierten Diagnosen dar. Demnach sind Stimuluskontrolle, Relaxationsübungen und kognitiv-behaviorale Therapie individuell wirksame Therapien zur Behandlung der chronischen primären Insomnie und Schlafrestriktion, Multikomponententherapie ohne kognitive Therapie, Biofeedback und paradoxe Intention individuell wirksame Therapien zur Behandlung der chronischen sekundären Insomnie.

Wenn mit den bereits beschriebenen Methoden zwei Drittel der Insomniepatienten geholfen werden kann (Hajak et al. 1997), dann leidet ein weiteres Drittel weiterhin an Insomnie. Da aus dargelegten Gründen (s. Kapitel 1.1.7.1) für diese Patienten die Pharmakotherapie keine befriedigende dauerhafte Lösung bieten kann, wird in der vorliegenden Studie die für Insomniepatienten neue Interpersonelle Psychotherapie untersucht und diese in ihrer Wirksamkeit mit der bereits bewährten Progressiven Muskelrelaxation nach Jacobson verglichen. Beide Behandlungsformen werden in den folgenden Kapiteln ausführlich dargestellt. 


\subsection{Interpersonelle Psychotherapie}

\subsubsection{Einleitung}

Ein langfristiger durchschlagender Erfolg in der Therapie primärer Insomnien ist bisher ausgeblieben. Die Arousal-Theorie (s. Kapitel 1.1.4) bietet hierfür einen Erklärungsansatz: Während des Tages internalisierte Gefühle führen nach dem Zubettgehen zu einer emotionalen Erregung. Diese Erregung zieht wiederum eine körperliche Aktivierung nach sich, und dieses physiologische Arousal verhindert letztendlich das Einschlafen (Kales et al. 1984). Arousals sind nach den Kriterien der American Sleep Disorders Association (ASDA 1992) Kennzeichen für Schlafstörung, die ein schädliches Merkmal darstellen (Halasz et al. 2004). Die ungarischen Autoren sehen im physiologischen Arousal zwei Typen von Mikroarousals und sprechen ihnen unterschiedliche Ausprägungen vegetativer Aktivierung zu. Sie vermuten, dass die Arousals den Schläfer mit der Umgebung verbinden, damit er sich an von außen kommende Gefahren und Anforderungen anpassen kann.

Die bisher angewandten nichtmedikamentösen Therapieformen setzen nicht direkt an der Wurzel des Schlafproblems an: die Entspannungsverfahren zielen auf die Minderung des physiologischen Arousals ab. Einen früheren Ansatzpunkt nützen kognitive und Verhaltenstherapien: Die dem physiologische Arousal vorausgehende geistige Erregung wird z.B. durch den Gedankenstop aus der Einschlafphase verdrängt oder durch die kognitive Kontrolle in die Tageszeit vorverlagert (s. Kapitel 1.1.7.2.3).

Die Ursachen dieser geistigen Erregung jedoch wurden bisher noch nicht therapeutisch angegangen: Insomniepatienten haben sehr oft gravierende Probleme im zwischenmenschlichen Bereich oder in der Bewältigung einschneidender Lebensereignisse (s. Kapitel 1.1.4), die häufig mit Trauer oder Rollenwechsel einhergehen. Diese Ursachen primärer Insomnie sind der Ansatzpunkt einer neuen Psychotherapieform, der Interpersonellen Psychotherapie für Insomnie (IPT-I).

Die IPT ist als ambulante Kurzzeittherapie für Patienten mit unipolarer, nichtpsychotischer Depression entwickelt worden (Klerman et al. 1984), die einen Zeitraum von 12-20 Sitzungen umfasst. Ihre ersten Ansätze aus den frühen 70er Jahren wurden im Laufe der letzten 25 Jahre immer weiter verbessert. „Der Ansatz der IPT ist in internationalen Leitlinien empfohlen und zählt $\mathrm{zu}$ den am besten überprüften Depressionsbehandlungen“ (Schramm 2007, S.362). Für die Depression und weitere psychische Erkrankungen sind Modifikationen entstanden. Die IPT basiert zum einen auf der Theorie A. Meyers (1957), der psychische Krankheit betrachtete als den Versuch 
einer Person, sich an eine sich verändernde Umwelt anzupassen, zum anderen auf der interpersonellen Schule Sullivans (1953) und seiner Mitarbeiter mit der Theorie über den Zusammenhang von psychiatrischen Krankheiten und interpersonellen Beziehungen.

\subsubsection{Der interpersonelle Kontext}

Gestörte interpersonelle Beziehungen können einerseits zur Ausbildung einer psychischen Störung beitragen, zum anderen hat eine psychische Störung Auswirkungen auf die Beziehungen eines Menschen zu seinen nächsten Bezugspersonen. Am Beispiel der Depression wird diese Wechselwirkung im Folgenden näher erläutert. Einschneidende Lebensereignisse wie der Verlust einer nahestehenden Person durch Tod oder Trennung können soziale Beziehungen gravierend verändern und depressiogene Wirkung haben (Brown und Harris 1978, Brown et al. 1986). Der Verlust oder die Gefahr des Verlustes von sozialen Bindungen führt zu Angst und Traurigkeit und kann die Entstehung einer Depression begünstigen (Bowlby 1969). Hingegen wirken gute soziale Beziehungen schützend gegen die Entwicklung einer Depression (Brown et al. 1980). Ein Fürsorge hervorrufendes Verhalten kann soziale Unterstützung fördern, wird diese Unterstützung nicht ausreichend gewährt, kann dieses Verhalten sich ins Krankhafte steigern und zur Depression führen, die zunächst erhöhte soziale Unterstützung hervorruft (Henderson 1977). Auf der anderen Seite hat eine Depression deutliche Auswirkungen auf die interpersonellen Beziehungen eines Patienten. In einer ausschließlich an Frauen durchgeführten Untersuchung zum Beispiel stellte sich heraus, dass Depressive bei Gesunden Gefühle von Feindseligkeit, Depressivität und Ängstlichkeit hervorrufen und von ihnen negativer beurteilt werden als gesunde Frauen (Coyne 1976). Nicht nur interpersonelle Schwierigkeiten, sondern auch Probleme mit der Erfüllung sozialer Rollen stehen sowohl mit Depressionen als auch mit Schlafstörungen in einem wechselseitigen Zusammenhang. Nach der Chicagoer Schule (Klerman et al. 1984) erwächst das Ich aus den Interaktionen zwischen dem Kind und seinen Bezugspersonen, das erwachsene Ich wird durch Beziehungen zwischen dem Individuum und seiner Umgebung erhalten. Die strukturgebenden Elemente dieser Beziehungen sind die sozialen Rollen, von denen jedes Individuum mehrere ausübt. Nach dem interpersonellen Ansatz beeinflussen sich soziale Rolle und Krankheit gegenseitig: Probleme bei der Erfüllung sozialer Rollen können zu klinischer Psychopathologie führen, andererseits behindert die Krankheit wiederum die Erfüllung sozialer Rollen (Klerman et al. 1984). 
Drei Faktoren sind nicht nur für die Ausbildung der Depression, sondern auch für den Beginn einer Schlafstörung von Bedeutung: Einschneidende, vor allem unangenehme oder mit Verlust verbundene Lebensereignisse, Schwierigkeiten in interpersonellen Beziehungen und ein negatives Selbstbild (s. Kapitel 1.1.4). Insomniepatienten haben mehr zwischenmenschliche Konflikte als Kontrollpersonen (Vollrath et al. 1989), insbesondere negative Lebensereignisse stehen in unmittelbarem Zusammenhang mit Schlafstörungen (Cernovsky 1984). Zeitlich ist dabei vor allem das Jahr vor Beginn der Insomnie relevant, das mehr negative Lebensereignisse beinhaltet als vorhergehende oder nachfolgende Jahre (Healey et al. 1981). Der Verlust einer Beziehung spielt eine besondere Rolle. Gefragt nach ihrer Kindheit berichten Insomniepatienten eher als Kontrollpersonen von wenig zufriedenstellenden Beziehungen und häufigeren somatischen Problemen. Das Selbstbild der Betroffenen ist deutlich negativer als das guter Schläfer: Sie sehen sich selbst eher als passiv, schwach, ungenügend, unkontrolliert, weniger anpassungsfähig und unklug. Außerdem haben sie mehr Schwierigkeiten in interpersonellen Beziehungen. Es fällt ihnen schwerer, befriedigende soziale Beziehungen aufrechtzuerhalten (Healey et al. 1981). Schlafgestörte sehen sich wie Depressive ungenügend und inkompetent und glauben, von ihrer Umgebung ebenso negativ eingeschätzt zu werden (Klerman et al. 1984). Auch internalisieren sie ihre Schwierigkeiten, um dann mit somatischen Problemen und sozialem Rückzugsverhalten zu reagieren (Kales et al. 1976, 1983). Retrospektiv beschreiben sich Insomniepatienten als ängstlich, angespannt und besorgt (Coursey et al. 1975). Sie sind eher introvertiert, weniger aggressiv als gute Schläfer und pessimistisch (Engel und Knab 1985).

\subsubsection{IPT für Insomnie (IPT-I)}

Aufgrund dieser Fülle möglicher interpersoneller Probleme lag es nahe, die IPT in einer modifizierten Version auch für Schlafgestörte anzuwenden. Das entsprechende Manual stammt von E. Schramm (1993 unveröffentlicht, zit. n. Müller-Popkes 1997, S.8). Zuvor war die IPT schon für spezifische Aspekte der Depression sowie für andere psychische Störungen abgeändert worden (s. Kapitel 1.2.4).

Die IPT für Insomnie (IPT-I) ist der ursprünglichen IPT recht ähnlich. In unserer Studie ist sie auf 12 Stunden begrenzt. Wie bei der IPT für Depressive liegen die Ziele der IPTI in der Symptombewältigung und in der Bewältigung der interpersonellen Schwierigkeiten, die mit der Insomnie in Zusammenhang stehen (Schramm 1993 unveröffentlicht, zit. n. Müller-Popkes 1997, S.69-70). Die Störung wird nicht einfach als Folge dieser zwischenmenschlichen Probleme, sondern als eingebettet in den interpersonellen Kontext verstanden. Die IPT für Insomniepatienten strebt ebensowenig 
wie die IPT für Depressive eine Veränderung der Persönlichkeit an. Die Krankheiten unterscheiden sich in der zeitlichen Dynamik: Schlafstörungen remittieren langsamer und verlaufen weniger phasenhaft als die Depression. Die Schlafstörung hat häufig seit vielen Jahren bestanden und sich verfestigt, bis die Patienten sich in Behandlung begeben (Simen et al. 1995). Daher nimmt die Symptombewältigung bei der IPT-I einen größeren Raum ein als bei der Urform der IPT, wo sie nach den ersten Sitzungen thematisch beendet wird. Die Patienten müssen zum Teil ihre Verhaltensweisen ändern, Gewohnheiten aufgeben und neue Verhaltensweisen einüben. So wird nicht nur in den ersten Sitzungen viel Wert auf das Symptommanagement gelegt, auch in den späteren Sitzungen wird immer wieder das Schlaf-Wach-Verhalten kontrolliert und gegebenenfalls weiter daran gearbeitet (Müller-Popkes 1997).

Die IPT-I besteht aus drei Therapiephasen, die nach einem kurzen Überblick ausführlich dargestellt werden. In der ersten Phase wird der Patient nach einer eingehenden Anamnese über seine Diagnose und den Schlaf informiert und über Schlafhygiene aufgeklärt. Dann erfolgt eine Bestandsaufnahme der wichtigen sozialen Beziehungen und deren Zusammenhang mit der Schlafstörung. In der zweiten Phase wird der zwischenmenschliche Problembereich bearbeitet, der ursächlich oder aufrechterhaltend mit der Schlafstörung in Zusammenhang steht. Die dritte Therapiephase thematisiert das Ende der Therapie als eine Zeit des Abschieds und der möglichen Trauer und zieht Bilanz über das Erreichte.

\section{Die ersten Sitzungen}

In den Anfangssitzungen werden die Anamnese erhoben und aktuelle Beschwerden, frühere Therapieversuche und weitere Erkrankungen erfragt. Die Patienten erfahren so, dass ihre Symptome ernst genommen werden und dass sie eine therapierbare Krankheit haben. Die eingehende Anamnese trägt darüber hinaus dazu bei, andere Erkrankungen als Ursache der Schlafstörung auszuschließen. Zuletzt erhält der Patient Informationen über seine Krankheit und die Behandlungsmöglichkeiten.

Zur schlafhygienische Abklärung werden gezielte Fragen gestellt, um die Schlafstörung aufrechterhaltende Verhaltensweisen zu erfassen:

1. Ist der Schlaf-Wach-Rhythmus des Patienten sehr unregelmäßig oder zeitlich verschoben?

2. Verrichtet der Patient im Schlafzimmer sowohl tagsüber als auch nachts mit dem Schlaf unvereinbare Tätigkeiten wie arbeiten, essen, fernsehen? 
3. „Hat der Patient negative Gedanken oder Gefühle bezüglich des Schlafes oder des Schlafzimmers? Geht er ängstlich zu Bett, weil er fürchtet, nicht schlafen zu können? Grübelt der Patient viel im Bett? Liegt der Patient lange wach im Bett, um verlorenen Schlaf nachholen zu wollen?“ (Müller-Popkes 1997, S. 71)

4. „Tragen fehlangepasste Einstellungen zur Schlafstörung bei? Hat der Patient Vorurteile über den Schlaf? Versucht er, seine Schlafstörung mit schlafinkompatiblen Mitteln zu fördern (z.B. viel Sport vor dem Schlafengehen, Alkohol als Schlafhilfe)?“ (Müller-Popkes 1997, S. 71)

5. „Gibt es andere Störquellen, die zur Schlafstörung beitragen, wie z.B. Lärm, Temperatur und Helligkeit des Schlafzimmers?“ (Müller-Popkes 1997, S. 71)

6. „Wird die Schlafstörung durch das Tagesgeschehen verschlimmert? Ist der Patient tagsüber ausgelastet, oder ist er überanstrengt?“ (Müller-Popkes 1997, S. 71)

Schlafgestörte Patienten haben häufig einen unregelmäßigen Tag-Nacht-Rhythmus mit wechselnden Schlafzeiten, wenig Tagesstrukturierung und viel Aktivität in den Abendstunden. Nach der sozialen Zeitgeber-Hypothese besteht eine enge wechselseitige Beziehung zwischen interpersoneller Belastung und Unterbrechung der sozialen Rhythmen im Leben des Patienten (Ehlers et al. 1988). Soziale Zeitgeber sind z.B. zwischenmenschliche Beziehungen oder soziale Pflichten, die den biologischen Rhythmus aufrechterhalten. Auch belastende Lebensereignisse können zu einer Verschiebung der Tag-Nacht-Struktur führen. Während sich normalerweise diese Verschiebung allmählich wieder einpendelt, kann es bei vulnerablen Menschen zu einer Destabilisierung verschiedener biologischer Rhythmen, wie z.B. dem des Schlafes (Healey et al. 1981), kommen. Durch sensorische Deprivation kann es zu einem besonderen Zustand kommen, der in der Dynamik von Herzschlagfrequenz und der Dynamik des EEG dem REM-Schlaf ähnelt (Iwata et al. 2000).

In dieser Studie wird der Tag-Nacht-Rhythmus mit der sozialen Rhythmusskala (Frank et al. 1990a unveröffentlicht, zit. n. Müller-Popkes 1997, S.71-72) genauer erfasst, um ihn gegebenenfalls ändern zu können. Alle Aktivitäten des Tages mit dem Grad der Beteiligung anderer Personen werden dort eingetragen. Der Patient füllt über mindestens drei Wochen täglich diese Skala aus, in der er notiert, welche Aktivitäten er wann unternimmt, auch die Tagesstimmung wird dort vermerkt. Dabei hält der Patient während dieser Beobachtungsphase an seinem gewohnten Tagesablauf fest. Nach dieser Zeit besprechen Patient und Therapeut die Protokolle und versuchen, Unregelmäßigkeiten und ihre Ursachen zu finden. In einem vorläufigen Plan halten sie Veränderungen fest, die für den gesunden Schlaf nötig sind. Eine realistische Planung 
ist dabei von großer Bedeutung. Während der nächsten Wochen versucht der Patient, durch die Umsetzung des Planes einen besseren sozialen Rhythmus zu erreichen. Der Erfolg des Patienten bei dieser sozialen Rhythmisierung wird während der gesamten Therapie überprüft, dabei kann die ursprüngliche Planung gegebenenfalls modifiziert werden (Müller-Popkes 1997).

Der Patient wird nach der Diagnosestellung ausführlich über den Schlaf und schlafhygienische Maßnahmen beraten. Förderliche und hinderliche Verhaltensmaßnahmen werden erklärt, Vorurteile und Ängste des Patienten hinsichtlich möglicher Folgen der Schlafstörung abgebaut. Die Informationsvermittlung ist sehr wichtig in der Therapie, weil viele Patienten sich aus Unkenntnis über den normalen Schlaf und seine Variationen häufig unnötig sorgen. Grundsätzlich ist eine medikamentöse Behandlung neben der IPT-I möglich. Der Therapeut sollte Vor- und Nachteile individuell abwägen und mit dem Patienten einen genauen Zeitraum der Medikamentengabe absprechen, nachdem er ihn über die Risiken, Nebenwirkungen und Absetzphänomene aufgeklärt hat. In dieser Studie wurde auf die Kombination der IPT-I mit Schlafmedikation verzichtet, da die Ergebnisse sonst nicht eindeutig zuzuordnen gewesen wären (Müller-Popkes 1997).

Nach der Befunderhebung und der Informationsvermittlung bezüglich des Tag-NachtRhythmus und der Schlafhygiene wendet sich die Therapie dem interpersonellen Inventar zu: Alle relevanten sozialen Beziehungen und ihr Zusammenhang mit der Schlafstörung werden erfasst bzw. bereits im Rahmen der Symptomerhebung Erfragtes vervollständigt. Dafür verschafft der Therapeut sich einen Überblick über alle gegenwärtigen und vergangenen Beziehungen seines Patienten innerhalb der sozialen Gruppen. Wichtig sind die Einschätzungen des Patienten von sich und anderen, der gegenseitigen Rollen und der Entwicklung der Beziehungen, seine Normen, Erwartungen und die Bedeutung, die er Rollen und deren Erfüllung zuschreibt sowie die ganze Palette damit verbundener Gefühle (Klerman et al. 1984).

Die IPT-I arbeitet ausdrücklich gegenwartsbezogen, vergangene Beziehungen werden zwar erfasst, sind jedoch nicht Gegenstand der Therapie.

Oft fällt es Patienten schwer, einen Zusammenhang zwischen der Schlafstörung und den zwischenmenschlichen Problemen zu erkennen. Die Beziehungen, Veränderungen im sozialen Rhythmus und belastende Ereignisse müssen deshalb sehr genau und ausführlich analysiert werden, um auslösende und aufrechterhaltende Faktoren der Insomnie zu ermitteln. Schlafgestörte sind häufig auf eine mögliche organische Ursache 
ihrer Erkrankung fixiert und leugnen zugrundeliegende Probleme. Der Therapeut ist wie in der IPT nicht neutral, sondern steht auf der Seite des Patienten und verhält sich besonders in den ersten Sitzungen aktiv und direktiv. Die therapeutische Beziehung gilt als Modell für andere Beziehungen, es gilt wie für die anderen Formen der IPT: Übertragungsphänomene werden nur thematisiert, wenn sie für die Therapie hinderlich sind oder gar ein Abbruch der Therapie durch den Patienten droht (Schramm 2007).

Während der Exploration hat der Therapeut herausgearbeitet, welchem Problembereich die Schwierigkeiten zuzuordnen sind. Im Anschluss wird mit dem Patienten ein Therapievertrag aufgesetzt, in dem realistische Ziele bezüglich der Symptombewältigung und der interpersonellen Probleme formuliert werden, das ist für die Erhöhung der Compliance sehr bedeutsam (s. Kapitel 1.1.7.2.5). Ein oder zwei Problembereiche werden ausgewählt und zum Gegenstand der folgenden Sitzungen erklärt. In diesem Vertrag wird auch festgehalten, dass der Patient das Material zur Bearbeitung liefert (Müller-Popkes 1997).

\subsubsection{Die Bearbeitung des Problembereichs}

Folgende vier Problembereiche können behandelt werden:

Trauer

interpersonelle Konflikte mit wichtigen Personen aus dem privaten oder beruflichen Bereich, sei es der Ehepartner oder der Geliebte, seien es die Kinder, Freunde oder Arbeitskollegen

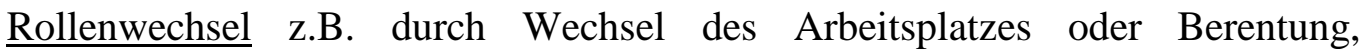
Scheidung, Umzug oder Veränderung familiärer oder wirtschaftlicher Verhältnisse

interpersonelle Defizite, aufgrund derer der Patient einsam und sozial isoliert ist (Klerman et al. 1984)

Weitere Problembereiche sind möglich.

\subsection{Trauer}

Normale Trauerreaktionen wie Traurigkeit, Schlafstörung, Agitiertheit und die verminderte Fähigkeit, tägliche Verrichtungen auszuführen, verlieren sich nach zwei bis vier Monaten (Klerman et al. 1984).

Fehlt dieser normale Trauerprozess, kann eine anomale Trauerreaktion die Folge sein, die zur Depression oder Schlafstörung führt. Diagnostische Hinweise können der Schilderung des Patientin entnommen werden: eine zeitliche Häufung mehrerer Todesfälle, die Aufrechterhaltung der Umgebung wie vor dem Tod oder die Angst vor 
der Krankheit des Verstorbenen können Anzeichen einer anomalen Trauerreaktion sein (Müller-Popkes 1997).

Eine verzögerte Trauerreaktion kann zum Ausbruch kommen, wenn der Patient an den nicht verarbeiteten Tod erinnert wird, z.B. durch einen aktuellen, an sich weniger belastenden Todesfall. Eine verzerrte Trauerreaktion kann sowohl sofort als auch Jahre nach dem Tod einer geliebten Person auftreten und sich weniger in Traurigkeit und Dysphorie als vielmehr in nichtaffektiven Symptomen äußern, die von oft zahlreichen konsultierten Ärtzten nicht ursächlich mit der Trauer in Verbindung gebracht werden (Schramm und Berger 1994).

Bei der Anamnese der interpersonellen Beziehungen des Patienten fragt der Therapeut daher auch nach früheren wichtigen Beziehungen mit später verstorbenen Personen.

Therapieziel ist es, den Trauerprozess zu erleichtern. Dazu schildert der Patient ausführlich die Beziehung mit dem Verstorbenen und seine Gefühle vor, während und nach dessen Tod. Der Therapeut hilft so dem Patienten, starke Gefühle und Ängste (Horowitz 1976) zu artikulieren und auch negative Gefühle gegenüber dem Verstorbenen zuzulassen, ohne deren Bearbeitung der Trauerprozess sich nicht vollziehen kann. Nach der Aufarbeitung der Beziehung unterstützt der Therapeut den Patienten bei der Öffnung gegenüber anderen Menschen und dem Aufbau und der Pflege von Beziehungen, die den Verlust ersetzen können (Klerman et al. 1984).

\subsection{Interpersonelle Rollenkonflikte}

Interpersonelle Rollenkonflikte entstehen, wenn der Patient und eine oder mehrere für ihn wichtige Personen unterschiedliche Erwartungen an ihre Beziehung haben. Dieser Rollenkonflikt kann vor allem dann für die Auslösung der Schlafstörung verantwortlich sein, wenn er dauerhaft ist und der Patient sich unfähig sieht, den Konflikt zu entschärfen, wenn es an Kommunikation mangelt und der Patient das Ende der Beziehung und das drohende Alleinsein fürchtet (Klerman et al. 1984). Häufig neigen Patienten in der Therapie dazu, interpersonelle Auseinandersetzungen zu bagatellisieren. Anzeichen hierfür kann sowohl eine idealisierte Darstellung der Beziehung als auch die Vermeidung des Themas sein.

Ziel der Behandlung ist es, dem Patienten bei der Identifizierung des Rollenkonfliktes zu helfen. Es ist für das weitere Vorgehen wichtig zu klären, ob sich die Beziehung noch im Verhandlungsstadium befindet, ob sie in eine Sackgasse geraten ist oder ob sie sich in Auflösung befindet (Schramm 1993 unveröffentlicht, zit. n. Müller-Popkes 1997, 
S.58). Erst dann kann der Therapeut zusammen mit dem Patienten einen Aktionsplan entwickeln und ihn dazu anhalten, falsche Kommunikationsmuster und gegebenenfalls auch seine Erwartungen zu ändern, um den Rollenkonflikt lösen zu können (Klerman et al. 1984). Liegt die Lösung in einer Trennung, ähnelt die weitere Therapie der Erleichterung des Trauerprozesses (s. Kapitel 1.2.3.1.1).

\subsection{Rollenwechsel}

Jeder Mensch durchlebt mehrere Rollenwechsel, zum größten Teil solche, die einen neuen Lebensabschnitt einleiten, wie z.B. das Verlassen des Elternhauses, der Beginn der Elternschaft oder des Ruhestandes. Durch Angst vor, Unzufriedenheit mit oder das Gefühl des Versagens in einer neuen Rolle kann sich sowohl eine Depression als auch eine Schlafstörung ausbilden. Auch wenn eine Person sich der Notwendigkeit eines Rollenwechsels bewusst ist aber damit Schwierigkeiten hat, kann eine Störung auftreten, oder aber, wenn die Person ein Versagen in einer Rolle richtig erkennt, aber nicht bereit oder fähig ist, ihr Verhalten oder ihre Rolle zu ändern. Der Verlust des familiären Rückhalts, der Erwerb neuer sozialer Kompetenzen und die Bewältigung von den Rollenwechsel begleitenden Gefühlen wie Angst oder Ärger sind die häufigsten Probleme, die mit dem Rollenwechsel verbunden sind (Klerman et al. 1984).

Nachdem der Therapeut zunächst herausgearbeitet hat, dass die Schlafstörung Folge eines Rollenwechsels ist, hilft er dem Patienten, alle Vor- und Nachteile der alten und der neuen Rolle zu erkennen. Ähnlich wie im Trauerprozess ermöglicht er ihm, Gefühle wie Wut, Schuld und Angst zuzulassen, um sich von der alten Rolle und damit verbundenen Beziehungen lösen zu können. Faktoren, die den Patienten an der Erfüllung der neuen Rolle gehindert haben, müssen erkannt und bearbeitet werden, dazu zählen auch falsche Vorstellungen und überhöhte Leistungsansprüche an sich selbst.

\subsection{Interpersonelle Defizite}

Finden sich im interpersonellen Inventar überwiegend vergangene unbefriedigende Beziehungen und ist der Patient zum Zeitpunkt der Therapie weitgehend sozial isoliert, so ist der Problembereich der interpersonellen Defizite das Aktionsfeld des Therapeuten. Das langfristige Ziel der Therapie ist, die Isolation zu beenden und dauerhafte Beziehungen zu schaffen, in der kurzen Zeit können die interpersonellen Defizite jedoch nur in Ansätzen abgebaut werden. Der Therapeut verhält sich hier aktiver als in den übrigen Problembereichen, da ein Patient mit bedeutenden interpersonellen Defiziten kaum in der Lage ist, die Therapie aktiv mitzugestalten. Mangels gegenwärtiger Beziehungen werden frühere analysiert. Das Training 
zwischenmenschlicher Kommunikation geschieht im Rahmen der Therapeut-PatientBeziehung, die besonders bei diesem Problembereich als Modell dient.

\subsubsection{Beendigung der Therapie}

Der Therapeut spricht in den letzten Sitzungen das bevorstehende Ende der Therapie an und gibt dem Patienten Gelegenheit, Gefühle und Befürchtungen zu äußern und diesen Abschied als eine Zeit des Trauerns zu verstehen. Fortschritte des Patienten bei der Symptombewältigung und der Gestaltung interpersoneller Beziehungen werden bewertet, Erfolg und noch bestehende Defizite besprochen sowie Möglichkeiten, letztere zu beheben. Dem Patienten wird sowohl das Vertrauen in die eigene Kompetenz vermittelt als auch das Erkennen ihrer Grenzen, damit er beim Auftreten erneuter nicht zu bewältigender Probleme rechtzeitig professionelle Hilfe sucht (Müller-Popkes 1997). In den verbleibenden Sitzungen wird die Tag-Nacht-Rhythmisierung weiter gefestigt.

\subsubsection{Die Effektivität der IPT und ihrer Modifikationen}

Die Wirksamkeit der IPT für Depressive ist im Vergleich mit Medikamenten und einer kognitiven Therapie mehrfach überprüft worden. 1979 nahmen 81 ambulante depressive Patienten an einer kontrollierten Studie teil. Sowohl IPT als auch Amitriptylin erwiesen sich signifikant effektiver als die Kontrollgruppe, eine Kombinationsbehandlung war jedoch allen überlegen (Weissman et al. 1979, DiMascio 1979). Das soziale Funktionsniveau der mit IPT und der Kombinationsbehandlung therapierten Patientengruppen war gegenüber der Medikamenten- und der Kontrollgruppe signifikant erhöht. Zehn Jahre später wurde in der NIMH-Studie zur Depressionsbehandlung (Elkin et al. 1989) die IPT im Vergleich mit einer kognitiven Therapie, mit Imipramin und mit einer stützende Gespräche erhaltenden Kontrollgruppe getestet. Nach der 16wöchigen Behandlung ging es den Patienten in allen Behandlungsgruppen deutlich besser, mehr als die Hälfte der 250 Patienten war symptomfrei. Wegen der starken Verbesserung in der Kontrollgruppe waren die Ergebnisse jedoch nicht signifikant. Bei der Behandlung der schwer erkrankten Patienten waren im Gegensatz zur kognitiven Therapie sowohl die IPT als auch Imipramin signifikant wirksamer als die Kontrollgruppe. Die IPT war bezüglich einzelner Messwerte ebenso wirksam wie die medikamentöse Therapie.

Auch die verschiedenen Abwandlungen der IPT sind auf ihre Wirksamkeit hin überprüft worden: Die Entwicklung einer Langzeitversion der IPT ergab sich zum einen aus der Tatsache, dass einige Patienten noch lange nach Remissionsbeginn Defizite in der sozialen Anpassung zeigen. Patienten mit wiederholt auftretenden depressiven Phasen, die sich schlecht behandeln lassen, brauchen ebenfalls eine kontinuierliche Behandlung 
über einen längeren Zeitraum. Es wurde die Hypothese aufgestellt, dass eine eingeschränkte soziale Funktionsfähigkeit einen Risikofaktor für eine erneute depressive Episode darstellt (Frank 1991, Frank et al. 1991). In der Erhaltungstherapie IPTMaintenance (IPT-M) werden die remittierten Patienten über etwa drei Jahre mit monatlichen Sitzungen weiterbehandelt. Es werden weitere Problembereiche und Probleme, die durch die Erkrankung oder deren Ende entstanden sind, bearbeitet. Den Übergang von der IPT zur IPT-Maintenance stellt ein Wechsel von wöchentlichen zu monatlichen Sitzungen dar. Die IPT-M wurde in einer Studie mit 5 Behandlungsgruppen überprüft (Frank et al. 1990b): 1. IPT-M allein, 2. IPT-M und Imipramin (ca. 200 mg), 3. IPT-M und Placebo, 4. stützende Gespräche und Imipramin und 5. stützende Gespräche und Placebo. Die Behandlung dauerte drei Jahre, wenn es nicht bereits zuvor zu einer erneuten depressiven Episode kam. Die Patienten der Imipramingruppen blieben längere Zeit beschwerdefrei als die der Psychotherapiegruppen und der Kontrollgruppe. Patienten, die mit IPT-M allein oder in Kombination mit Placebo behandelt wurden, hatten eine fast doppelt so lange beschwerdefreie Zeit wie die Patienten der Kontrollgruppe.

Es wurden weitere spezifische Modifikationen der IPT entwickelt. Für Patienten mit interpersonellen Auseinandersetzungen in der Paarbeziehung wurde die Paartherapie IPT-Conjoint marital (IPT-CM) entwickelt (Weissman und Klerman 1993), bei der die Depression auslösende und aufrechterhaltende Beziehungsmuster erfasst und verändert werden. Wie in der Ursprungsform der IPT werden nur die aktuellen Beziehungen bearbeitet. Bei dem Vergleich von IPT und IPT-CM (Foley et al. 1989) zeigten Patienten in beiden Gruppen eine deutliche Besserung der depressiven Symptomatik und der sozialen Funktionsfähigkeit, wobei sich die Gruppen in diesen Maßen nicht voneinander unterschieden. Die Interaktion zwischen den Partnern gestaltete sich in der Gruppe der IPT-CM-Patienten nach Beendigung der Therapie in mehreren Bereichen deutlich besser als in der IPT-Gruppe.

Weitere Therapieabwandlungen existieren für depressive Jugendliche (IPT for Adolescents, IPT-A; Mufson et al. 1993) und ältere Menschen (IPT-late life (IPT-LL), IPT-LLM als Erhaltungs- (Maintenance) Therapie der IPT-LL; Sholomskas et al. 1983, Frank et al. 1993). Als Kurzzeittherapie für spezielle Patientengruppen wie z.B. HIVpositive Depressive (Markowitz et al. 1993) kam die IPT ebenso erfolgreich zum Einsatz wie zur Behandlung der Bulimie (Fairburn et al. 1993). In letzterer müssen die Patientinnen zunächst lernen, dass ihr gestörtes Essverhalten Ausdruck zwischenmenschlicher Probleme ist, die bearbeitet werden. Das Essverhalten selbst 
steht in der Behandlung völlig im Hintergrund. Wie die Schlafstörung verläuft die Bulimie im Gegensatz zur Depression oft chronisch (Russel 1979, Herzog et al. 1991). In einer klinischen Studie erwies sich die IPT als ebenso erfolgreich wie die kognitivbehaviorale Therapie, die bisher vorwiegend in der Therapie von Bulimie-Patientinnen eingesetzt wird (Fairburn et al. 1991). Beide Therapien waren auch in der Katamnese nach zwölf Monaten erfolgreicher als eine rein behaviorale Therapie, die sich nur auf die Normalisierung des Essverhaltens konzentrierte. Bei der IPT dauerte der Eintritt einer Besserung zwar etwas länger, der Zustand der Patientinnen verbesserte sich jedoch noch während des Katamnesezeitraumes weiter. Es trat eine Besserung des Essverhaltens und der Selbstbeurteilung von Figur und Gewicht ein, obwohl diese Themen in der Therapie nicht behandelt wurden. (Fairburn et al. 1993)

Die IPT-Modifikationen für Suchtkranke (Rounsaville und Carroll 1993) wie die für Opiat-(Rounsaville et al. 1983, Woody et al. 1983) und Kokainsüchtige (Carroll et al. 1991) konnten sich nicht bewähren.

\subsection{Progressive Muskelrelaxation}

Die Progressive Muskelrelaxation (PMR) ist eines der am häufigsten angewandten verhaltenstherapeutischen Entspannungsverfahren. Die bereits in den dreißiger Jahren von Jacobson (1938) entwickelte Therapie findet seit den siebziger Jahren in unterschiedlich modifizierter Form ihre Anwendung (z.B. Bernstein und Borkovec 1973) und hat sich auch in der Therapie von Insomniepatienten bewährt. Jacobson (1938) erkannte den Zusammenhang von Muskelkontraktion, Spannungsgefühl und Angst und fand heraus, dass Angst durch den Abbau der Muskelspannung gemindert werden konnte. Durch das systematische Anspannen und Entspannen verschiedener Muskelgruppen und durch die Konzentration darauf kann es gelingen, sich sehr entspannt zu fühlen. Jacobsons Verfahren war mit insgesamt 56 Sitzungen sehr langwierig und wurde von späteren Anwendern modifiziert und verkürzt. Zunächst wandte Wolpe (1958) die so abgewandelte PMR im Rahmen der systematischen Desensibilisierung bei Angstpatienten an. Wie Jacobson sah auch er die Entspannung als Gegensatz zur ängstlichen Anspannung. Die PMR setzte sich im Laufe der Zeit immer mehr durch und wurde im Rahmen der Angstbehandlung und gegen eine Vielzahl anderer Störungen eingesetzt. Zur Behandlung der Insomnie gab es zu Beginn der 70er Jahre erste kontrollierte Studien. Bernstein und Borkovec (1973, 1995) entwickelten das in Studien heute am meisten verwendete Verfahren, bei dem die Muskelgruppen mehrfach angespannt werden, bis der Patient sie als entspannt 
empfindet. Die PMR ist umso wirksamer, je verspannter die Patienten zu Therapiebeginn sind (Hauri 1981).

Über die Wirkungsweise der PMR gibt es unterschiedliche Theorien (Nicassio und Bootzin 1974, Haynes et al. 1974, Borkovec et al. 1975, Borkovec et al. 1979, Borkovec und Hennings 1978, Woolfolk und McNulty 1983). Die Arbeitsgruppe um Borkovec hält die tatsächliche Entspannung der Muskeln für entscheidend und schreibt der in der progressiven Relaxation bewussten Wahrnehmung der körperlichen Vorgänge sogar einen negativen Einfluss zu (Borkovec und Hennings 1978). Als nicht entscheidend für den Therapieerfolg beurteilten dagegen Woolfolk und McNulty (1983) die An- und Entspannungsübungen. In ihrer Studie über vier Wochen zeigte sich die Richtung der Aufmerksamkeit auf neutrale Gegenstände als wirksamer als die Muskelentspannungsformen PMR und die um die Anspannungsphase verminderte somatische Fokussierung. Freedman und Papsdorf (1976) konnten keine signifikante Beziehung zwischen dem Grad der physiologischen Aktivierung und der Einschlaflatenz herstellen. Es gab in der Studie jedoch Anzeichen für eine gesteigerte kognitive Aktivierung: Die Patienten berichteten von Gedanken, die das Einschlafen verhinderten. Dazu gesellt sich nach klinischer Erfahrung eine Schlaferwartungsangst (Espie 1991), die diese geistige Erregung noch weiter verstärkt. Durch die regelmäßige Anwendung der PMR können die Patienten ihre kognitive Aktivität reduzieren und von den Gedanken, die das Einschlafen verhindern, ablenken.

\subsubsection{Die Durchführung der PMR}

Den relevanten Modifikationen der PMR ist gemeinsam, dass 16 Muskelgruppen nacheinander isoliert angespannt und entspannt werden. Die vorhergehende Anspannung ermöglicht eine tiefere Entspannung, die durch den Kontrasteffekt deutlicher wahrgenommen wird.

„Ablauf der Sitzungen: Der Patient sitzt oder liegt in bequemer Kleidung in einem möglichst lärm- und lichtgeschützten Raum“ (Müller-Popkes 1997, S. 79) und folgt den Anweisungen des Therapeuten. Er spannt die Muskeln zunächst für fünf bis sieben Sekunden an und entspannt sie danach für 30-45 Sekunden. Er soll während der Sitzung nicht sprechen. Der Therapeut erinnert den Patienten immer wieder an die Konzentration auf die entspannten Muskelpartien. Nach der Entspannung aller Muskelpartien führt der Therapeut den Patienten langsam in die Realität zurück. Er zählt dazu abwärts und spricht noch einmal Wohlbefinden und Entspannung an, bevor er den Patienten auffordert, sich zum Abschluss zu strecken und rekeln. Diese 
zunehmende körperliche Aktivierung am Ende eines Durchgangs unterbleibt jedoch, wenn der Patient vor dem Einschlafen übt.

Der Patient lernt das An- und Entspannen der Muskelgruppen in einer bestimmten Reihenfolge. „Folgende Muskelgruppen werden angespannt:

1. Dominante Hand und Unterarm

2. Dominanter Oberarm

3. Nichtdominante Hand und Unterarm

4. Nichtdominanter Oberarm

5. Stirn

6. Obere Wangenpartie und Nase

7. Untere Wangenpartie und Kiefer

8. Nacken und Hals

9. Brust, Schultern und obere Rückenpartie

10. Bauchmuskulatur

11. Dominanter Oberschenkel

12. Dominanter Unterschenkel

13. Dominanter Fuß

14. Nichtdominanter Oberschenkel

15. Nichtdominanter Unterschenkel

16. Nichtdominanter Fuß“

(Müller-Popkes 1997, S. 80).

Manche Autoren schlagen vor, die einzelnen Muskelpartien zweimal anzuspannen und zu entspannen. Außerdem können die Muskelpartien mit anderen, bereits entspannten Gruppen in ihrem Entspannungsgrad verglichen werden (Bernstein und Borkovec 1995). 


\subsubsection{Effektivität der PMR bei Patienten mit primärer Insomnie}

Die PMR ist bereits vor Jahrzehnten mit verhaltenstherapeutischen und mit anderen Entspannungsmethoden verglichen worden. Polysomnografisch kontrollierte Studien der Wirksamkeit bei Insomniepatienten sind rar, meist wurde lediglich die subjektive Einschlaflatenz anhand von Schlaftagebüchern erfasst, seltener wurden weitere Schlafparameter untersucht. Es deutet sich in den kontrollierten Studien von Borkovec und Fowles 1973 sowie von Nicassio und Bootzin 1974 eine Abhängigkeit des Behandlungserfolges und des Placeboeffektes an vom Schweregrad der Schlafstörung, von der Behandlungsdauer und von der Erwartungshaltung der Patienten.

Um herauszufinden, in welchem Ausmaß die Wirksamkeit der PMR auf unspezifische Effekte beruht, ließen Steinmark und Borkovec (1974) die Patienten die Glaubwürdigkeit der jewiligen Behandlungsform bewerten und ihnen mitteilten, dass bis zur vierten und letzten wöchentlichen Sitzung keine Besserung des Zustandes zu erwarten sei (Counterdemand). Beim Vergleich von PMR, systematischer Desensibilisierung, einer Quasidesensibilisierung (Placebo) und einer Wartelistenkontrollgruppe zeigten in dieser Untersuchung nur die PMR und die systematische Desensibilisierung schon vor der letzten Sitzung signifikante Wirkung, jedoch lediglich auf die Einschlaflatenz. Bis zur Nachuntersuchung nach fünf Monaten verstärkte sich diese noch weiter. In der letzten Sitzung auftretende Erfolge der Placebogruppe konnten sich dagegen nicht halten.

In einer längeren Studie verglichen Nicassio et al. (1982) PMR mit EMG-Biofeedback, Biofeedback-Placebo und einer Wartelistenkontrollgruppe über zehn Sitzungen. Dabei zeigte sich in der subjektiven täglichen Beurteilung eine kontinuierliche Abnahme der Einschlaflatenz bei der PMR, dem Biofeedback und dem Biofeedback-Placebo, die Placebogruppe war den beiden Therapien unterlegen. Andere Schlafparameter erfuhren keine signifikante Besserung.

Im Vergleich mit der Stimuluskontrolle zeigte diese sich in mehreren Studien gegenüber der PMR in der Reduktion der Einschlaflatenz wirksamer (Bootzin 1975 unveröffentlicht, Lawrence und Tokarz 1976, zit. n. Espie 1991, S. 178-180; Lacks et al. 1983a)

Die PMR ist bei klinischen Stichproben einer Placebo- oder Kontrollgruppe überlegen, wobei die Verbesserung des Schlafes vorwiegend auf der subjektiven Einschätzung beruht. Die Wirkung der PMR beschränkt sich hauptsächlich auf die signifikante Verringerung der Einschlaflatenz, es wurden jedoch auch Auswirkungen auf die 
Schlafqualität (Espie et al. 1989), die Aufwachqualität (Lick und Heffler 1977) und die Anzahl nächtlicher Aufwachvorgänge (Haynes et al. 1974) beobachtet. Nach bisherigen Studien unterscheidet sich die PMR in ihrer Wirksamkeit nicht wesentlich von anderen Entspannungsverfahren. Im Vergleich zu weiteren psychologischen Therapien scheint nur die Stimuluskontrolle der PMR hinsichtlich der Einschlaflatenz überlegen zu sein (Lacks et al. 1983a,b), es gibt aber auch Studien, die dies nicht bestätigen können (Turner und Ascher 1979). Während sich die Wirkung von kurz dauernden PMRTherapien auf die Verbesserung der Einschlaflatenz beschränkt, beeinflussen längere Behandlungen zusätzlich die Schlafqualität positiv (Turner und Ascher 1979, Espie et al. 1989, Lacks et al. 1983a).

\subsection{Das Ziel dieser Untersuchung}

Das Ziel der dieser Untersuchung zugrunde liegenden Studie war herauszufinden, ob die IPT-I eine wirksame Behandlungsmethode für Patienten mit primärer Insomnie ist. Dazu wurde sie mit der bereits mehrfach erprobten und bewährten PMR verglichen. Neben der positiven Wirkung auf den Schlaf waren auch Tagesbefindlichkeit, Lebenszufriedenheit und die Verbesserung interpersoneller Beziehungen von Interesse. Diese Bereiche hätten jedoch den Rahmen dieser Untersuchung gesprengt und sind Gegenstand anderer Arbeiten (Kesten i.V., Müller-Popkes 1997).

Die vorliegende Untersuchung beschäftigt sich ausschließlich mit den Auswirkungen der angewandten therapeutischen Verfahren auf den Schlaf im Verlauf der Behandlung. Die Patienten beurteilen das Ausmaß ihrer Schlafstörung weniger anhand der im Schlaflabor ermittelten objektiven Parameter, sondern hauptsächlich nach ihrer subjektiven Einschätzung der Schlafquantität und -qualität. Diese subjektiven Beurteilungen waren daher von größerem Interesse als die Polysomnografiewerte, zumal verschiedene Therapieformen auf letztere nur einen geringen Einfluss hatten (Espie 1991).

Aus den in Müller-Popkes 1997 veröffentlichten Daten war zu ersehen, dass die IPT-I im Vergleich zur PMR für die meisten Patienten keine wesentliche Besserung der Schlafstörung bringt (Müller-Popkes 1997). Die Patienten hatten jedoch mehr Fragebögen ausgefüllt, als in bisherigen Auswertungen berücksichtigt wurden. Es sollte nun unter Berücksichtigung eines weiteren Fragebogens, der bislang keinen Eingang in die Auswertung fand, überprüft werden, ob sich hierdurch deutliche Veränderungen sichtbar machen lassen. In der vorliegenden Untersuchung wurde dafür bislang unveröffentlichtes Datenmaterial berücksichtigt, das während der Aufenthalte im 
Schlaflabor vor und nach der Therapie gewonnen wurde und Informationen über den subjektiven Schlaf enthielt.

Es sollten zudem objektive den subjektiven Parametern gegenübergestellt werden, um mögliche therapiebedingte Veränderungen der Abweichungen qualitativ erfassen zu können.

Des weiteren liegen bislang noch keine Kenntnisse über die Veränderungen der subjektiven Schlafbewertung während der zwölfwöchigen Therapie vor. Es wurden bislang nur Daten veröffentlicht über die Baseline, die Zeitpunkte der Schlaflabornächte vor Beginn und nach Beendigung der zwölfwöchigen Behandlungen sowie zu den Nachbeobachtungen drei und sechs Monate nach Behandlungsende (Müller-Popkes 1997). Um zu untersuchen, ob in den während der zwölfwöchigen Behandlungen stattfindenden Veränderungen der Beurteilungen des Schlafes durch die Patienten eine mögliche Ursache für das geringe Ausmaß der Therapieeffekte liegt, sollten die während der gesamten Behandlung durch die Patienten täglich durchgeführten Beurteilungen ihres Schlafes im Verlauf betrachtet werden.

Aufgrund des hohen zeitlichen Aufwands insbesondere der IPT-I (Müller-Popkes 1997) sollte geprüft werden, ob auch durch eine verkürzte Therapie Behandlungserfolge zu erzielen waren. Eine ursprünglich niedriger angesetzte Altersobergrenze sollte hier Berücksichtigung finden und deshalb der in der bisherigen Auswertung Extremwerte liefernde älteste Patient eliminiert werden, um ein deutlicheres Bild der Therapieeffekte nicht zu verwischen.

Zudem war es nun durch die über die bisherige Auswertung (Müller-Popkes 1997) hinaus zusätzlich ermittelten und analysierten Daten, die Auskunft über den subjektiven Schlaf während der zwölfwöchigen Behandlung geben, zu folgendem Erkenntnisgewinn gekommen: Die subjektive Bewertung der Polysomnografienächte wich von der subjektiven Beurteilung der häuslichen Nächte stark ab. Deshalb sollte die Frage nach einer Verkürzungsmöglichkeit der Behandlungsdauer unter Berücksichtigung der Beurteilung der in der gewohnten häuslichen Umgebung verbrachten Nächte beantwortet werden. Hierzu wurde weiteres, bislang unveröffentlichtes Datenmaterial ausgewertet, das Informationen zur täglichen Beurteilung des Schlafes während der laufenden Behandlung liefert. 


\subsubsection{Annahmen über die Veränderungen des subjektiven Schlafes und ihre Konsequenzen für die Auswertung}

\subsubsection{Schlafquantität}

Da die Stichprobe sich vor allem aus Patienten zusammensetzte, die entweder an Einoder an Durchschlafstörungen litten, war zu erwarten, dass sich nur die jeweils entsprechenden quantitativen Schlafparameter verbessern. Zwar müsste die Besserung beider Schlafstörungen zu einer Zunahme der Gesamtschlafzeit führen, bei einer Verteilung des Therapieerfolgs auf die zwei Gruppen Ein- und Durchschlafgestörte wären in den statistischen Tests für die entsprechenden quantitativen Parameter jedoch nicht unbedingt signifikante Ergebnisse zu erwarten. Bereits die Änderung der Hälfte der quantitativen Schlafparameter wäre somit als Therapieerfolg zu werten.

\subsubsection{Schlafqualität}

Die Datenerhebung der qualitativen Schlafparameter durch jeweils einen Test der Schlafqualität und einen Test des morgendlichen Erholungsgefühls ist ebenfalls zu differenziert, um bei der vorliegenden Patientenzahl in beiden Tests signifikante Ergebnisse liefern zu können. Auch hier kann deshalb eine signifikante Änderung jeweils der Hälfte der untersuchten qualitativen Parameter als Therapieerfolg betrachtet werden.

\subsubsection{Bewertungszeitraum}

In der Gesamtbetrachtung eines längeren Zeitraumes bewerten Patienten ihren Schlaf schlechter als bei kurzfristiger Beurteilung (Mendelson 1987). Daher wurden die unterschiedlichen Bewertungszeiträume getrennt untersucht. Da sich der Schlaf in der Einschätzung durch den Patienten theoretisch auch verschlechtern kann, erfolgten auch hier die Tests zweiseitig.

\subsection{Die Bewertung des Schlafes der zurückliegenden zwei Wochen}

Für die Beurteilung des Schlafes der vergangenen zwei Wochen wurde untersucht, ob die Patientengruppen vor Behandlungsbeginn miteinander vergleichbar sind.

Patienten neigen dazu, ihre schlechten Nächte eher im Gedächtnis zu behalten als die guten Nächte. Sie bewerten daher ihren Schlaf über einen längeren Zeitraum als schlechter als in der kurzfristigen Beurteilung (Dement et al. 1984). Fragebögen, die sich auf die zurückliegenden zwei Wochen beziehen, lassen deshalb generell eine negativere Beurteilung des Schlafes erwarten als Protokolle einer vergangenen Nacht. Es war jedoch zu erwarten, dass sich bei beiden Gruppen in den Beurteilungen der 
Schlafparameter eine Besserung zum posttherapeutischen Messzeitpunkt gegenüber dem Messzeitpunkt vor Behandlungsbeginn ergeben würde. Da sich der Schlaf theoretisch in der subjektiven Bewertung auch unter der Therapie verschlechtern könnte, erfolgen die statistischen Tests zweiseitig.

Damit auch eine unterschiedliche Veränderung der längerfristige Bewertung des Schlafes im Laufe der Behandlung beider Gruppen erfasst werden kann, wurden mögliche Interaktionseffekte berücksichtigt.

\subsection{Der Schlaf in der täglichen Bewertung}

Auch in der täglichen Beurteilung wurde eine Vergleichbarkeit der Patientengruppen vor Behandlungsbeginn überprüft.

Es war zu erwarten, dass sich bei beiden Gruppen in den allmorgendlichen Beurteilungen der Schlafparameter eine deutliche Besserung im Verlauf der Behandlung ergeben würde. Auch hier ist wegen möglicher negativer Auswirkungen der Behandlungen eine zweiseitige Testung nötig.

Zur Prüfung eines möglichen Interaktionseffektes wurde hier genauso wie bei der Bewertung eines zweiwöchigen Zeitraums untersucht, ob sich in einer der beiden Gruppen die Einschätzung der vergangenen Nacht signifikant stärker ändert als in der anderen Guppe.

\subsubsection{Der Schlaf nach zwei Dritteln des Behandlungszeitraumes}

Es sollte untersucht werden, ob ein Therapieerfolg bereits acht Wochen nach Behandlungsbeginn eintritt. Wegen möglicher negativer Auswirkungen nach acht Wochen erfolgt die Testung zweiseitig.

Zur Prüfung eines möglichen Interaktionseffektes wurde untersucht, ob sich in einer der beiden Gruppen die Einschätzung der vergangenen Nacht schon nach acht Wochen signifikant stärker als in der anderen Gruppe verändert. 


\section{Material und Methoden}

\subsection{Patienten}

Das Patientengut ist mit der in Müller-Popkes 1997 verwendeten Stichprobe identisch. Die detaillierte Darstellung wurde bereits veröffentlicht (Müller-Popkes 1997) und wird in diesem Abschnitt noch einmal dargelegt.

In Zeitungsartikeln und einer Radiosendung war über die geplante Studie berichtet worden. Sowohl durch die Information in den Medien als auch durch die Schlafambulanz der Klinik erkundigten sich die Patienten telefonisch nach der Behandlung. 61 der Patienten erschienen zu einem Vorgespräch im Schlaflabor, wo sie einen Aufklärungsbogen und eine Einwilligungserklärung unterschrieben und damit die Bereitschaft bestätigten, sich an vereinbarte Termine zu halten, auf Medikamente zu verzichten und in der Therapie aktiv mitzuarbeiten.

Die Geschlechtsverteilung war in beiden Gruppen gleich. Die Altersgrenzen wurden auf 18 und 70 Jahre festgelegt. Eine geplante Begrenzung der Krankheitsdauer auf drei Jahre konnte nicht umgesetzt werden, da die meisten Patienten erst nach jahrelanger chronischer Erkrankung ins Schlaflabor kommen (vgl. Simen et al. 1995).

Im Erstgespräch wurden die Patienten nach körperlichen Erkrankungen, Medikamentenund Alkoholkonsum, psychischen Störungen und organisch bedingten Schlafstörungen gefragt. Sie schilderten genau ihre Symptome und deren zeitlichen Verlauf.

Nach den Kriterien der bei Studienbeginn gängigen Klassifikationssysteme ICD-10 (WHO 1991) und DSM-III-R (APA 1987) gab die subjektive Empfindung insomnischer Beschwerden den Ausschlag für die Aufnahme in die Studie. Patienten mit einer positiven Suchtanamnese wurden nicht aufgenommen. Psychopathologische Auffälligkeiten von Krankheitswert sind mit der Diagnose „primäre Insomnie“ unvereinbar, vor allem Depressionen und Angsterkrankungen mussten ausgeschlossen sein. Die Abgrenzung der primären Insomnie von einer möglichen Dysthymie erfolgte anhand der Symptomatik zu Beginn der Schlafbeschwerden.

Körperliche Erkrankungen, die die Insomnie verursacht haben oder sie beeinflussen können, sind ebenfalls nicht mit einer primären Insomnie in Einklang zu bringen. Während des stationären Aufenthalts erfolgte deshalb neben der Schlafableitung eine 
ärztliche internistische und neurologische Untersuchung sowie EKG und EEG, die Erfassung der Routinelaborparameter, einschließlich der Schilddrüsenparameter, der Luesserologie und der Blutsenkung. Erbrachten die Untersuchungen auffällige Befunde wie zum Beispiel den Hinweis auf eine Schilddrüsenfehlfunktion, wurden die betroffenen Patienten nicht in die Studie aufgenommen, sondern gegebenenfalls über die Notwendigkeit einer diagnostischen Abklärung informiert.

Während der Studie durften die Patienten weder Schlafmedikamente einnehmen noch Präparate, die Schlafstörungen verursachen können. Falls ein Patient Schlafmittel einnahm, wurden diese bis zum Studienbeginn, das heißt bis zur Baseline kontrolliert ausgeschlichen. Weitere Vormedikation wurde mit der behandelnden Therapeutin besprochen (Müller-Popkes 1997).

Mit 42 Patienten wurde im diagnostischen Vorgespräch ein Termin zur Polysomnografie vereinbart. Die übrigen 19 Patienten wurden aus unterschiedlichen Gründen nicht in die Studie aufgenommen: Drei Patienten lehnten eine Behandlung ab, zwei von ihnen erschien ein Verzicht auf Schlafmedikation nicht möglich. Drei Patienten litten nicht an einer Schlafstörung. Vier Patienten hatten schwerwiegende organische Erkrankungen oder nahmen schlafstörende Medikamente ein. Neun Patienten litten an einer psychiatrischen Erkrankung, darunter vier an einer Depression, zwei an einer Persönlichkeitsstörung, zwei an einer Suchterkrankung, und eine Patientin litt an einer Angsterkrankung.

Die nach dem Erstgespräch durchgeführte Polysomnografie diente zum einen der Objektivierung der subjektiven Beschwerden, zum anderen dem Ausschluss organischer Ursachen. Wurden diese zugrundeliegenden organischen Störungen wie das Schlafapnoe- und das PMS-Syndrom aufgedeckt, führte dies zum Ausschluss der betroffenen Patienten aus der Studie und zu ihrer adäquaten Weiterbehandlung (s. Kapitel 2.3.2). Es stellte sich bei der Polysomnografie heraus, dass von den 42 Patienten nur 31 an einer primären Insomnie litten. Von den übrigen elf Patienten litten drei an einem Hypopnoe-Syndrom und acht an einem Syndrom nächtlicher periodischer Beinbewegungen. Diese Patienten wurden nicht in die Studie aufgenommen, sondern einer entsprechenden Behandlung zugeführt.

Zu Beginn der Studie bestand die Stichprobe aus 31 Patienten. Sechs Patienten brachen die begonnene Behandlung ab, so dass lediglich 25 Patienten die Behandlung vollständig durchführten und zur abschließenden Polysomnografie erschienen.

21 der 25 Patienten erschienen zu den Katamnesen nach drei und sechs Monaten. 
Alter, Erkrankungsdauer, Familien- und Bildungsstand und die berufliche Tätigkeit der 25 Patienten sind innerhalb der Gruppen sehr unterschiedlich, die beiden Gruppen sind jedoch bezüglich der genannten Variablen sowie der Geschlechtsverteilung im Wesentlichen vergleichbar.

Im Folgenden wird die 13 IPT-I-Patienten und zwölf PMR-Patienten umfassende Stichprobe genauer beschrieben. In der IPT-I-Gruppe waren fünf Männer und acht Frauen, in der PMR-Gruppe jeweils sechs Männer und Frauen. Die Geschlechtsverteilung unterschied sich nicht signifikant. Die Patienten waren 28 bis 66 Jahre alt, im Durchschnitt 47 Jahre. Die Dauer der Schlafstörung war mit einer Spanne von 0,5 bis 35 Jahren sehr unterschiedlich, sie betrug durchschnittlich in der IPT-IGruppe zehn Jahre und in der PMR-Gruppe 8,4 Jahre. Zweiseitige t-Tests für unverbundene Stichproben ergaben, dass sich die Gruppen bezüglich Alter und Erkrankungsdauer nicht signifikant voneinander unterschieden (Müller-Popkes 1997).

Die Patienten waren überwiegend verheiratet ( $n=17$, IPT-I: PMR=8:9), drei ledig (3:0), drei geschieden (0:3) und zwei lebten von ihrem Partner getrennt (2:0). Bis auf eine Patientin aus jeder Gruppe lebten alle mit einem Partner zusammen. Elf Patienten hatten einen Realschulabschluss (5:6), neun Patienten hatten Abitur (5:4), davon hatten vier ein Fachhochschul- oder Hochschulstudium absolviert (4:0). Fünf Patienten hatten einen Hauptschulabschluss (3:2). Zwei Patientinnen waren Hausfrau, eine arbeitslos, ein Patient war Rentner, drei befanden sich in einer Ausbildung und die übrigen waren berufstätig, elf in Vollzeit und sieben in Teilzeit.

Die Gruppen unterscheiden sich nicht signifikant bezüglich des Vorhandenseins eines festen Partners, ihrer Schulbildung und ihres Berufes, lediglich der Familienstand unterscheidet sich signifikant: In der IPT-I-Gruppe sind mehr Patienten ledig oder getrennt lebend, in der PMR-Gruppe sind mehr geschieden (Müller-Popkes 1997).

\subsection{Studienaufbau}

Sowohl die Interpersonelle Psychotherapie für Insomniepatienten (IPT-I) als auch die Progressive Muskelrelaxation (PMR) wurden durch dieselbe diplomierte Psychologin durchgeführt. In ihrer Dissertation (Müller-Popkes 1997) ist der in diesem Unterkapitel dargestellten Studienaufbau ausführlich beschrieben. Auf eine Wartelistenkontrollgruppe wurde verzichtet, da zum einen die progressive Muskelrelaxation nach Jacobson ein bereits auch für Schlafstörungen vielfach erprobtes 
Verfahren war, zum anderen es ethisch nicht vertretbar erschien, den hilfesuchenden Patienten für die Dauer der Therapie eine Behandlung vorzuenthalten.

Vor Studienbeginn war ein Probedurchgang mit jeweils einer Patientin pro Behandlungsgruppe vorgenommen worden, dabei hatte sich der Studienablauf als problemlos durchführbar erwiesen. Die Patienten wurden nun randomisiert den beiden Behandlungsgruppen zugeteilt. In einem Erstgespräch wurde ihnen zunächst der Ablauf der Studie erklärt, bevor sie die Einwilligungserklärung unterschrieben (s. Kapitel 2.1). Dann wurden die Krankheitsgeschichte und die Symptomatik erfasst, wobei auf die Abgrenzbarkeit zu anderen psychischen Störungen besonders geachtet wurde. Etwa zwei Wochen nach dem Erstgespräch fand die erste polysomnografische Untersuchung (PSG) (s. Kapitel 2.3.2) über zwei Nächte statt. In den zwei Wochen dazwischen wurde anhand der Visuellen Analogskalen (Ott et al. 1981) jeden Morgen die Schlafqalität erfasst (s. Kapitel 2.3.4).

Die Patienten füllten zu den Polysomnografienächten die Schlaffragebögen SF-A und SF-B (Görtelmeyer 1981) sowie den VIS-M (Ott et al. 1981) zur Beurteilung der Schlafqualität und -quantität aus. Diejenigen Patienten, für die sich durch die Polysomnografie die Diagnose der primären Insomnie bestätigt hatte (s. Kapitel 2.3.2), nahmen an zwölf wöchentlichen Einzelsitzungen teil, jeweils für 50 Minuten in der IPTI-Gruppe und für 30 Minuten in der PMR-Gruppe.

Während des gesamten Behandlungszeitraumes füllten die Patienten täglich die Fragebögen VIS-M aus. Zu für die Patienten unvorhersehbaren Terminen gaben sie Urinproben ab (s. Kapitel 2.3.5). In der Woche nach der zwölften Sitzung wurden die Patienten erneut zur Polysomnografie für zwei Nächte stationär aufgenommen. Im Schlaflabor füllten sie die gleichen Fragebogen aus wie bei der Erstuntersuchung.

Der polysomnografische Befund wurde mit den Patienten besprochen. Alle Patienten bekamen das Angebot, sich auch mit weiteren Schlafproblemen an die Therapeutin zu wenden. Mit den Patienten der IPT-I-Gruppe wurde vereinbart, sich während der sechs Monate nach Behandlungsende in keine anderweitige Psychotherapie zu begeben.

Drei und sechs Monaten nach Behandlungsende wurden die Patienten erneut zu einem Ambulanzgespräch in die Psychiatrische Klinik eingeladen, zu dem sie mit ausgefüllten Fragebögen erscheinen und zu ihrer Befindlichkeit und Schlafentwicklung Stellung nehmen sollten. Die zu diesen Nachbeobachtungsterminen erhobenen Daten sind jedoch nicht Gegenstand der vorliegenden Untersuchung. 
Neben den oben angeführten Fragebögen füllten die Patienten die folgenden Bögen aus, die jedoch nicht Gegenstand dieser Untersuchung sind: den Pittburgher Schlafqualitätsindex PSQI (Buysse et al. 1989), den Quality of Life of Insomniacs Questionnaire (QOLI) (Rombaut et al. 1990) in der deutschen Übersetzung, den abends auszufüllenden Teil der Visuellen Analogskalen zur Erfassung der Schlafqualität VIS-A (Ott et al. 1981), das Inventar zur Erfassung interpersonaler Probleme - deutsche Version IIP-D (Horowitz et al. 1994), die Depressions-Skala DS (v. Zerssen 1976), das Freiburger Persönlichkeitsinventar - revidierte Fassung FPI-R (Fahrenberg et al. 1984) und den Fragebogen zur Beurteilung der Behandlung FBB (Müller-Popkes 1997).

\subsection{Untersuchungsverfahren}

\subsubsection{Strukturiertes Interview für Schlafstörungen (SIS-D)}

In Erstgesprächen durch die Therapeutin wurden die Schlafstörungen der einzelnen Patienten standardisiert erfasst. Das Strukturierte Interview für Schlafstörungen (SIS-D) (Schramm et al. 1991) ermöglicht eine Diagnose von Schlafstörungen nach den Kriterien des DSM-III-R (APA 1987). In einer halben bis dreiviertel Stunde können auslösende Faktoren, die genaue Symptomatik und der zeitliche Verlauf der Erkrankung vollständig erfragt werden und zu einer vorläufigen Diagnose führen (Müller-Popkes 1997). Diese wird im Zweifelsfall noch durch weitere Untersuchungen wie zum Beispiel eine Polysomnografie erhärtet.

In einer multizentrischen Untersuchung zu den Gütekriterien des Interviews zeigen sich gute Werte zur Retest-Reliabilität (Schramm et al. 1990).

\subsubsection{Polysomnografie}

Die Polysomnografie (PSG) ist die lückenlose Aufzeichnung von Parametern während einer ganzen Nacht, um objektive Informationen über den Schlaf zu erhalten. Sie ermöglichte der Verfasserin mittels manueller Auswertung jeweils eines 30-SekundenZeitraumes eine Bestimmung der Schlafstadien nach den allgemein gültigen Kriterien von Rechtschaffen und Kales (1968).

Die Aufzeichnung geschah mit einem 22-Kanal-EEG-Gerät der Firma Nihon Kohden. Folgende Parameter wurden abgeleitet:

- Elektroenzephalogramm (EEG) (rechts zentrale bipolare Ableitung: C4 gegen A1) zur Bestimmung der verschiedenen Schlafstadien und des Wachzustandes, 
- Elektrookulogramm (EOG) zur beidseitigen Erfassung der Augenbewegungen, vorrangig zum Nachweis des REM-Schlafes

- Elektromyogramm (EMG) des M. mentalis zur Differenzierung zwischen REM- und Non-REM-Schlaf und zwischen Wachzustand und Schlaf anhand des Muskeltonus

- Elektrokardiogramm (EKG) (Ableitung Einthoven I oder II) und

- Elektromyogramm (EMG) der M. tibialis anterior rechts und links zur Kennzeichnung nächtlicher Beinbewegungen.

In der ersten Nacht wurden zur Überprüfung der Atmung zusätzlich

- nasaler und oraler Atemfluss mittels eines Drei-Wege-Thermistors der Firma Nihon Kohden,

- Thoraxatemexkursionen und Abdomenatemexkursionen, Messung durch Induktionspletysmographie, Gurte der Fima ZAK, und die

- Sauerstoffsättigung mit der Ohmeda Biox 3700e Pulsoximetrie am Finger gemessen.

Die Einstellung der Filter, Verstärkung und Zeitkonstanten erfolgte nach den allgemein üblichen Kriterien (Penzel et al. 1993) und wurde während der Nacht gegebenenfalls adaptiert, um eine möglichst genaue Aufzeichnung zu ermöglichen. Ausschlusskriterium für ein Schlafapnoe-Syndrom war ein Apnoe-/Hypopnoe-Index von mehr als 10/h (vgl. Lavie 1983), bei den nächtlichen periodischen Beinbewegungen während des Schlafes lag das Ausschlusskriterium bei mehr als 5/h.

Durch Eingabe der anhand der Polysomnografie ermittelten Schlafstadien in das schlaflaboreigene Auswertungsprogramm erhielt die Verfasserin eine grafische Darstellung der Schlafarchitektur (s. Anhang) und die Daten, auf deren Grundlage die Therapeutin zu den objektiven Schlafparametern die statistische Auswertung durchführte (Müller-Popkes 1997).

Zur Erfassung der objektiven Schlafparameter wurden Daten zu den vier Variablen Einschlaflatenz (EL), tatsächliche Schlafzeit (TST), Schlafeffizienz (SE) und nächtliches Wach (WASO) erhoben. Die Mittelwerte der zwei Nächte vor Behandlungsbeginn und die Mittelwerte der zwei Nächte nach Behandlungsende wurden für die einzelnen Gruppen bereits veröffentlicht (Müller-Popkes 1997). 


\subsubsection{Schlaffragebögen A und B (SF-A und SF-B)}

Während der Aufenthalte im Schlaflabor zur Eingangs- und Kontrollpolysomnografie machten die Patienten Angaben zu Schlafquantität und qualität. Da die subjektive Bewertung einzelner, isoliert betrachteter Nächte sich oft gravierend von der Einschätzung des Schlafs über einen längeren Zeitraum unterscheidet, wurden zwei verschiedene Schlaffragebögen SF-A und SF-B (Görtelmeyer 1981) ausgefüllt. Den zeitlichen Bezugsrahmen bildet im SF-A die letzte Nacht, im SF-B der Zeitraum der vergangenen zwei Wochen.

Es werden Daten zur Zeit des Lichtlöschens, zu Einschlaflatenz, Dauer und Häufigkeit der Aufwachvorgänge und Gesamtschlafzeit erhoben und Erscheinungen wie Muskelzuckungen, Herzstiche und weitere körperliche Beschwerden erfasst. Beurteilt werden zudem die Schlafqualität und das Befinden vor dem Zubettgehen und nach dem Aufstehen. Durch die Aufsummierung einzelner Items werden fünf Faktorenwerte berechnet, von denen in dieser Untersuchung nur die Daten zur Schlafqualität (SQ) und zum Gefühl des Erholtseins nach dem Schlaf (GES), das in den folgenden Kapiteln als „morgendliches Erholungsgefühl“ (mE) referiert wird, ausgewertet wurden. Daten zu psychischer Ausgeglichenheit, Erschöpftheit am Abend und psychosomatischen Symptomen in der Schlafphase wurden nicht berücksichtigt.

Die Faktorenwerte SQ und GES (mE) sind mit Koeffizienten von 0.92 bis 0.83 sehr konsistent. Die Retestreliabilität ergab Koeffizienten von 0.68 für die SQ und 0.78 für das GES (mE); bezüglich der Validität korreliert die SQ im SF-B mit der ärztlichen Beurteilung der Schwere der Schlafstörung mit 0.63, für die Korrelation des protokollierten nächtlichen Aufwachens mit dem ärztlichen Urteil „Durchschlafstörung“ liegt der Koeffizient bei 0.78 (Görtelmeyer 1981).

\subsubsection{Visuelle Analogskalen zur Erfassung der Schlafqualität (VIS-M)}

Die Visuellen Analogskalen zur Erfassung der Schlafqualität ähneln in der Thematik dem Schlaffragebogen A (SF-A, Görtelmeyer 1981), unterscheiden sich jedoch in der Art der Datenerhebung und in der Auswertung. Die tägliche Protokollierung während der gesamten Therapie erfolgte mit den Visuellen Analogskalen zur Erfassung der Schlafqualität (Ott et al. 1981), in denen im Selbstbeurteilungsverfahren die vergangene Nacht quantitativ und qualitativ bewertet wird.

Morgens nach dem Frühstück markieren die Patienten mit einem Strich auf einer unskalierten Linie, ob sie sich eher frisch oder müde fühlen, auf einer weiteren Linie markieren sie den Grad der Schlafqualität. Sie tragen auch Daten ein zu: Zeit des 
Lichtlöschens, Schlaflatenz, Aufwachhäufigkeit, echte Schlafzeit, Aufwachzeit und berichten über mögliche Alpträume. Eine freie Beschreibung von möglichen besonderen Ereignissen während der Nacht ist ebenfalls möglich.

Zur Auswertung wird jedes Item gesondert betrachtet, die Analogskalen werden von der Verfasserin vom linken Pol aus abgemessen und kodiert. Es gibt Normen für schlechte und gute Schläfer, für präoperative Patienten und für mäßig und leicht schlafgestörte Patienten mittleren und höheren Lebensalters.

Zur Retestreliabilität liegen Werte zu einzelnen Skalen vor. Die Werte schwanken zwischen 0.50 und 0.93 bei einem Zeitintervall von fünf bis zwölf Wochen, dabei liegen die meisten Koeffizienten zwischen 0.60 und 0.80 ; bezüglich der Validität liegen Angaben zur Schlafqualität in Relation zur Schlaflatenz und Durchschlafzeit vor. Die Koeffizienten liegen zwischen 0.25 und 0.80. (Ott et al. 1981)

Im VIS-M wurden täglich kontinuierlich während des gesamten Behandlungsverlaufes Daten zu den Variablen Einschlaflatenz, tatsächliche Schlafzeit, Schlafqualität und Aufwachqualität (morgendliches Erholungsgefühl) erhoben.

Die Daten und statistischen Auswertungen der gemittelte Werte der folgenden fünf Messzeiträume wurden bereits durch die Therapeutin Müller-Popkes 1997 veröffentlicht: 1. Baseline = BL (14 Fragebögen), 2. Prä = Eingangspolysomnografie (zwei Fb), 3. Post = Kontrollpolysomnografie: (zwei Fb), 4. $\mathrm{K} 3=$ Dreimonatskatamnese: (sieben Fb), 5. K6 = Sechsmonatskatamnese (sieben Fb).

Darüber hinaus wurden für die vorliegende Untersuchung die Daten von jeweils fünf Fragebogen zu den jeweils vor den Sitzungsterminen 2 (das heißt der ersten eigentlichen Therapiesitzung) bis 12 liegenden Nächten zu Mittelwerten zusammengefasst. Diese wurden in der vorliegenden Untersuchung für die Verlaufsbetrachtung während der Behandlung grafisch aufbereitet, sowie zum Teil zur Bearbeitung der Fragestellung einer möglichen Therapieverkürzung statistisch ausgewertet.

\subsubsection{Urinkontrollen}

Der Verzicht auf Schlafmedikamente während der Behandlung wurde anhand von Urinkontrollen überprüft. Diese wurden zu für die Patienten nicht vorhersehbaren Zeitpunkten jeweils im Anschluss an die Sitzungen durchgeführt. Die Urinproben wurden im Labor der Klinik semiquantitativ auf den Nachweis von Benzodiazepinen und Barbituraten untersucht. 


\subsection{Therapeutische Verfahren}

Die Anwendung der beiden Behandlungsmethoden in dieser Studie erfolgte durch ein und dieselbe Therapeutin. Sie gestaltete sich wie folgt:

\subsubsection{Interpersonelle Psychotherapie für Insomniepatienten}

Mit der Gruppe der IPT-I-Patienten wurde so verfahren, wie im Kapitel 1.2.3 beschrieben. Bereits in der ersten Sitzung verschaffte sich die Therapeutin einen Überblick über alle wichtigen sozialen Beziehungen und versuchte herauszufinden, welche mit der Auslösung oder Aufrechterhaltung der Insomnie in Zusammenhang standen. Die Therapeutin erklärte den Patienten dann den Therapieansatz und die zugrundeliegende Verknüpfung von Insomnie und interpersonellen Beziehungen (Müller-Popkes 1997).

Die zweite Sitzung war der Vermittlung von Informationen über gesunden Schlaf und schlaffördernde Verhaltensweisen sowie der Beratung und Aufklärung über schlafhygienische Maßnahmen gewidmet. Der Tagesablauf und das Verhalten am Abend und während der Nacht wurden genau besprochen. Anhand der Polysomnografieergebnisse konnte den Patienten ihre spezifische Schlafstörung erklärt werden. Die Patienten wurden gebeten, die Soziale Rhythmusskala (SRS) drei Wochen lang täglich auszufüllen, die anschließend besprochen wurden (Müller-Popkes 1997). Hierin wurde der Zeitpunkt bestimmter Aktivitäten des Tages notiert, um den Tagesablauf mit etwaigen dem Schlaf abträglichen Unregelmäßigkeiten zu ermitteln.

Die übrige Zeit war der Festlegung und Bearbeitung des interpersonellen Problembereiches gewidmet. Wie in Kapitel 1.2.3.1 beschrieben, wurden dazu die interpersonellen Beziehungen exploriert und mit der Insomnie in Verbindung gesetzt. Therapeutin und Patient einigten sich etwa am Ende der dritten Sitzung auf den Fokus der weiteren Therapie. Mit den einzelnen Patienten wurde ein individuelles Konzept entwickelt, um die fehlangepassten Verhaltensweisen verändern zu können. (MüllerPopkes 1997)

Die mittlere Therapiephase diente vor allem der Bearbeitung des Problembereiches. Das Schlafproblem rückte thematisch in den Hintergrund, der Patient wurde jedoch regelmäßig nach der Einhaltung der schlafhygienischen Regeln gefragt. Die letzte Phase der Therapie war der Beendigung des therapeutischen Prozesses und dem Ausblick auf noch bestehende Probleme gewidmet. Der Abschied von der Therapeutin wurde ebenfalls angesprochen und als ein Prozess der Trauer bearbeitet. (Müller-Popkes 1997) 
„Bei den Sitzungen zur Katamnese wurden die Patienten zu ihrem Schlaf befragt und wurden gebeten, von der Entwicklung der in der Therapie besprochenen interpersonellen Probleme zu berichten.“ (Müller-Popkes 1997, S. 111 f)

\subsubsection{Progressive Muskelrelaxation}

Die Gruppe der mit PMR behandelten Patienten bekam ebenfalls Informationen über gesunden Schlaf und schlafhygienische Regeln. „Da die einzelnen PMR-Sitzungen nur jeweils 30 Minuten dauerten, geschah die Vermittlung der Schlafhygiene in der zweiten und dritten Sitzung, um die Vergleichbarkeit mit der IPT-I-Gruppe zu gewährleisten.“ (Müller-Popkes 1997, S. 112). Nach der Demonstration ihres spezifischen Schlafes anhand des Polysomnogramms wurde mit den PMR-Patienten „der Tagesablauf und das Verhalten am Abend und während der Nacht besprochen. Die PMR-Patienten füllten jedoch keine Soziale Rhythmusskala aus“ (Müller-Popkes 1997, S. 112).

In der vierten Sitzung wurde den Patienten das Verfahren der progressiven Muskelentspannung erläutert. Ihnen wurden die Übungen erklärt und gezeigt mit dem Hinweis, dass sich die Wirkung erst nach einer gewissen Zeit „einstellen könne. Sie wurden gebeten, die jeweils in den Sitzungen durchgeführten Übungen zweimal täglich zuhause nachzuvollziehen, einmal während des Tages und einmal abends im Bett vor dem Schlafen“ (Müller-Popkes 1997, S. 112). Beginnend mit der Faust wurden nach und nach immer mehr Muskelgruppen hinzugenommen, bis in der neunten Sitzung alle Übungen demonstriert und geübt worden waren. Ein vollständiger Durchgang mit sämtlichen Muskelgruppen nimmt ungefähr 20 Minuten in Anspruch.

In den letzten drei Sitzungen wurden die Patienten „angeleitet, die Muskeln immer weniger anzuspannen und sich schließlich die An- und Entspannung nur noch vorzustellen. Ihnen wurde erklärt, dass sie nach einer Übungsphase dadurch den gleichen Effekt der Entspannung erzielen könnten“ (Müller-Popkes 1997, S. 113).

\subsection{Auswertung der Daten}

Für die objektiven Schlafparameter war durch die Verfasserin eine manuelle Auswertung der lückenlosen Schlafaufzeichnung nach Rechtschaffen und Kales im Halbminutentakt erfolgt und eine grafische Darstellung der Schlafarchitektur (s. Anhang) einschließlich der für die Berechnung der Schlafparameter erforderlichen Daten mittels des schlaflaboreigenen Auswertungsprogramms bereitgestellt worden. Anhand dieser Rohdaten waren von der Therapeutin die objektiven Schlafparameter 
ermittelt, die Gruppenmittelwerte der jeweiligen zwei Nächte vor und nach der Behandlung berechnet, statistisch ausgewertet und veröffentlicht worden (MüllerPopkes 1997). In der vorliegenden Untersuchung sind die Mittelwerte der Schlafparameter für die einzelnen Nächte als Gruppenmittelwerte aufgeführt (s. Kapitel 3.3).

Die mittels der Schlaffragebögen A und B (s. Kapitel 2.3.3) und der Visuellen Analogskala VIS-M (s. Kapitel 2.3.4) erhobenen Daten wurden mit dem Statistikprogramm SPSS für Windows Version 5.0.2. (Brosius und Brosius 1995) und mit dem Tabellenkalkulationsprogramm Excel 5.0 (Microsoft Corporation 1993) auf einem zweiseitigen Signifikanzniveau von $\alpha=0,05$ ausgewertet. Der unabhängige Faktor war mit den beiden Untersuchungsgruppen IPT-I und PMR zweistufig. Der abhängige Faktor bestand aus den Daten ausgewählter Messwiederholungen im Laufe der Behandlung. Die Auswertung von Intervall- und Ordinaldaten wird im Folgenden beschrieben und anschließend grafisch dargestellt (s. Abb. 2.01).

Intervalldaten wurden zum Teil mit mehrfaktoriellen univariaten Varianzanalysen (Bortz 1979) gerechnet. Diese dienen dazu, den Einfluss mehrerer unabhängiger und abhängiger Faktoren auf eine abhängige Variable zu untersuchen. Wenn abhängige Variablen auch inhaltlich miteinander verknüpft waren, wurden die betreffenden Werte der zu einer Fragestellung gehörenden Skalen eines Fragebogens in derselben Varianzanalyse gleichzeitig untersucht. In diesen mehrfaktoriellen multivariaten Varianzanalysen (Bühl und Zöfel 1996) wurden demnach mehrere abhängige Variablen gleichzeitig betrachtet. Im Anschluss an die signifikante Varianzanalyse wurde ein tTest für abhängige Stichproben gerechnet.

Ordinaldaten wurden, jede der betroffenen Skalen für sich, einem Mann-Whitney-UTest unterzogen, der überprüft, ob zwei unabhängige Verteilungen der gleichen Grundgesamtheit angehören. Anschließend wurden Wilcoxon-Tests zur Prüfung von Rangunterschieden bei wiederholten Messungen durchgeführt. Getestet wurde immer zweiseitig, da theoretisch nicht nur eine Besserung, sondern auch eine Verschlechterung möglich wäre. 
Auswertung der Daten

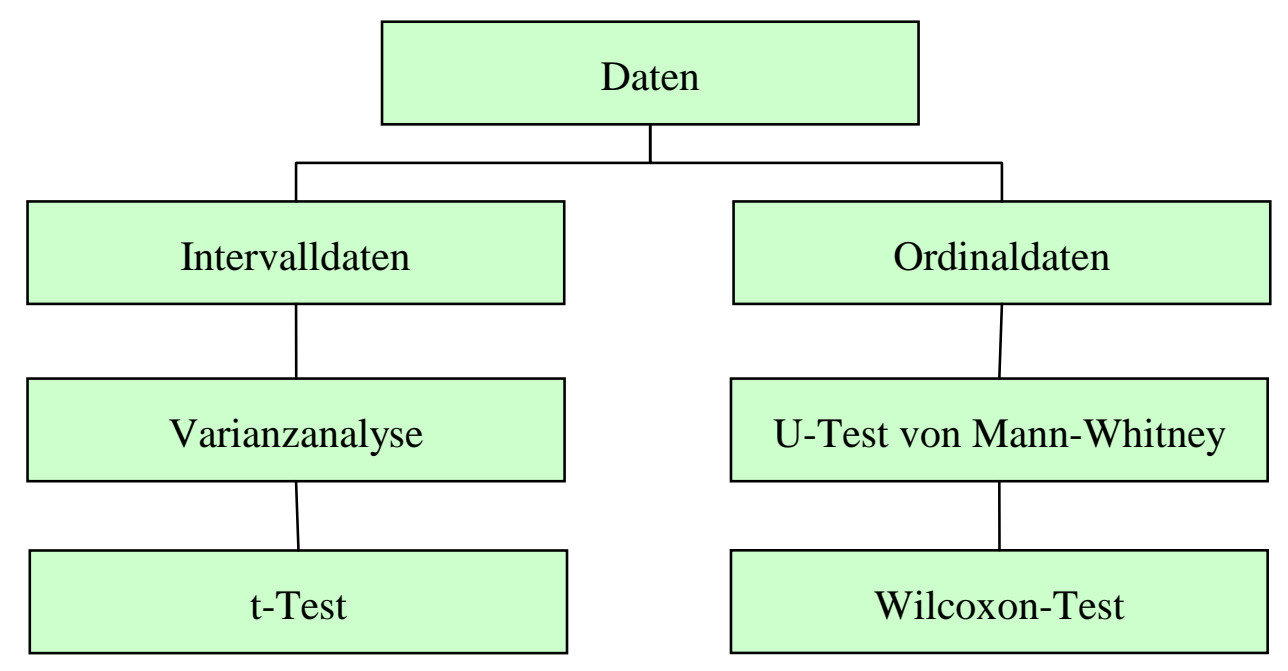

Abb. 2.01: Auswertung der Daten

Bei Fragestellungen, die Unterschiede von Schlafparametern prüften mithilfe von mehreren abhängigen Variablen für einen Schlafparameter, wurde eine BonferoniKorrektur durchgeführt: Um der Kumulation des alpha-Fehlers bei einer progressiven Fehlentscheidung entgegenzuwirken, wurde das Signifikanzniveau durch die Zahl der Einzeltests geteilt (Hager und Westermann 1983, Bortz et al. 1990). Bei Fragestellungen, die eine Vergleichbarkeit prüften, wurde zugunsten einer härteren Prüfung auf eine Fehleradjustierung verzichtet und zur Vermeidung einer konservativen Fehlentscheidung das Signifikanzniveau bei zweiseitiger Testung auf alpha=0,10 festgesetzt (Westermann und Hager 1982, Bortz et al. 1990). 
Die Daten des SF-A und des SF-B (s. Kapitel 2.3.3) wurden nur während der Aufenthalte der Patienten im Schlaflabor zu Beginn und nach Beendigung der Therapie erhoben, hier erfolgte ein Prä-Post-Vergleich mit den entsprechenden Mittelwerten (s. Abb. 2.02).

Auswertung der zu den Polysomnografieterminen

erhobenen Daten

Prä

Mittelwert der zu den

Eingangspolysomno-

grafieterminenn

erhobenen Daten
Post - Vergleich

Mittelwert der zu den Kontrollpolysomnografieterminen erhobenen Daten

Abb. 2.02: Auswertung der zu den Polysomnografieterminen erhobenen Daten des SF-A und des SF-B

Die quantitativen und qualitativen Aspekte des subjektiven Schlafes der vergangenen Nacht wurden anhand von Einzeltests des von der Verfasserin eingesetzten SF-A (s. Kapitel 2.3.3) und des VIS-M, dessen bereits von Müller-Popkes 1997 veröffentlichte Baseline-Daten im Kapitel „Ergebnisse“ gesondert gekennzeichnet werden, ermittelt. Die quantitativen und qualitativen Aspekte des subjektiven Schlafes eines zurückliegenden zweiwöchigen Zeitraumes wurden anhand von Einzeltests des von der Verfasserin eingesetzten SF-B (s. Kapitel 2.3.3) ermittelt.

Die VIS-M-Fragebögen wurden von der Verfasserin im Gesamtverlauf der Behandlung betrachtet. Von Interesse war zum einen die zeitliche Dynamik der Veränderung, zum anderen, ob schon vor Behandlungsende eine Besserung der täglichen Beurteilung des subjektiven Schlafes eintrat, oder ob sich bereits im Laufe der Therapie eingestellte Veränderungen zum Ende der Behandlung hin wieder verlieren. Jeweils fünf der den Behandlungsterminen zwei bis zwölf vorausgehenden Nachtprotokolle wurden zu einem Mittelwert zusammengefasst. Zum ersten Behandlungstermin, dem vor den Ableitungsnächten durchgeführten Erstgespräch, waren keine VIS-M-Bögen ausgegeben worden.

Ursprünglich war für die vierzehn VIS-M-Bögen der Baseline und die jeweils zwei Fragebögen der Eingangs- und Kontrollpolysomnografienächte jeweils ein Mittelwert berechnet worden, zusammen mit den Mittelwerten der Drei- und 
Sechsmonatskatamnesen waren diese fünf Messzeitpunkte bereits von der Therapeutin statistisch ausgewertet und veröffentlicht worden (Müller-Popkes 1997).

Für die vorliegende Untersuchung wurden aus der IPT-I-Gruppe nachträglich die VISM-Daten eines Patienten eliminiert, da er Extremwerte geliefert hatte. Dieser Patient war zugleich der älteste Patient beider Behandlungsgruppen.

\section{VIS-Verlaufsbetrachtung}

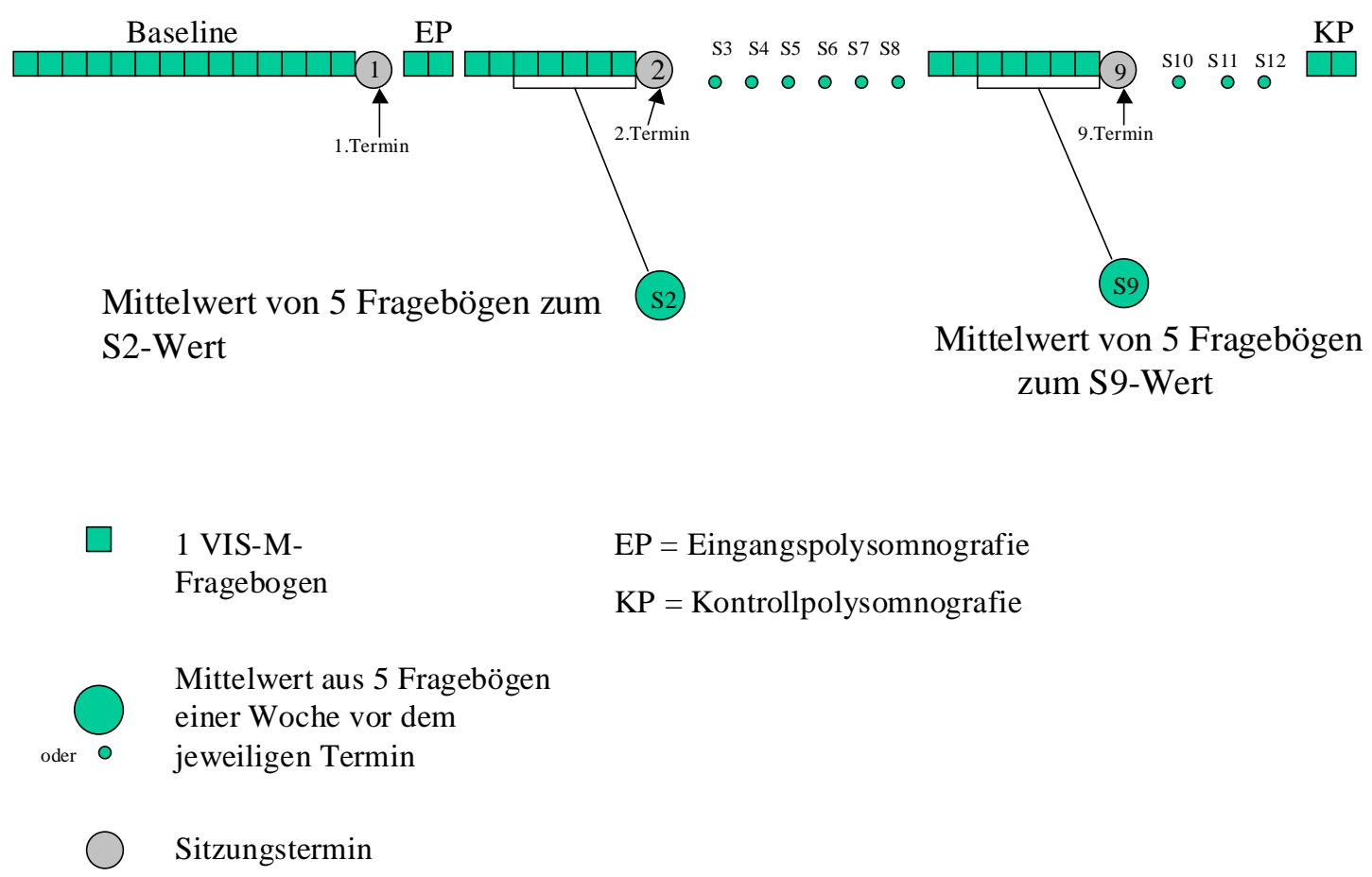

Abb. 2.03: VIS-M-Verlaufsbetrachtung

Um zu klären, ob sich die subjektiven Schlafparameter gegenüber der Baseline schon vor dem Ende der Behandlung verändern, wurden von der Verfasserin zu den Parametern tatsächliche Schlafzeit (TST), Einschlaflatenz (EL), Schlafqualität (SQ) und morgendliches Erholungsgefühl (mE) Varianzanalysen gerechnet. Da sich bei der Verlaufsbetrachtung der Mittelwerte der 15 Messzeiträume gezeigt hatte, dass sich die Beurteilungen der Ableitungsnächte im Schlaflabor nicht ideal in den Kurvenverlauf integrieren ließen, wurden diese Werte bei der anschließenden statistischen Berechnung nicht berücksichtigt.

Drei der 15 Messzeiträume fanden Eingang in die statistischen Berechnungen: 1. die nach dem Erstgespräch ermittelte Baseline (BL) (s. Kapitel 2.3.4, dieser Messzeitpunkt wurde bereits von Müller-Popkes 1997 veröffentlicht), 2. die Zeit vor der zweiten 
Sitzung (S2), das heißt der dem Erstgespräch und der Polysomnografie folgenden ersten eigentlichen Therapiesitzung sowie 3. die Zeit vor der neunten Sitzung (S9). Dieser letzte Messzeitraum wurde zur Klärung der Frage gewählt, ob sich die IPT-I auf acht Sitzungen verkürzen ließe, ohne an therapeutischer Wirksamkeit zu verlieren. 


\section{Ergebnisse}

Die eingesetzten Fragebögen SF-A und SF-B beschreiben die subjektive Beurteilung des Schlafes im 2-Wochen-Rückblick (SF-B) und im Rückblick auf die vergangene Nacht (SF-A) sowohl in quantitativer als auch in qualitativer Hinsicht zu zwei Zeitpunkten: vor und nach der Behandlung.

Der Verlauf der täglichen Einschätzung der vergangenen Nacht während des Zeitraums der Behandlung wird in dieser Untersuchung gesondert betrachtet, auch unter dem Gesichtspunkt, ob eine Verkürzung der Therapie möglich ist. Hierzu wurde der Fragebogen VIS-M eingesetzt.

\subsection{Patienten - Stichprobe}

Die Stichprobe bestand zu Beginn der Studie aus 31 Patienten, die randomisiert in eine IPT-I-Gruppe mit 15 Patienten und eine PMR-Gruppe mit 16 Patienten eingeteilt wurden.

Die begonnene Behandlung wurde von sechs Patienten aus unterschiedlichen Gründen vorzeitig abgebrochen.

25 Patienten führten die Behandlung vollständig durch und erschienen zur abschließenden Polysomnografie. Auf diese Stichprobe mit 13 Patienten in der IPT-IGruppe und zwölf Patienten in der PMR-Gruppe beziehen sich die ausgewerteten Daten. Da sich in der Verlaufsbetrachtung herausgestellt hatte, dass der mit 67 Jahren älteste Patient Extremwerte lieferte, gingen die Daten dieses Patienten aus der IPT-I-Gruppe in die Auswertungen zur Beurteilung einer möglichen Therapieverkürzung nicht mit ein.

+Urinkontrollen auf Benzodiazepine und Barbiturate waren mit einer Ausnahme unauffällig. Die betreffende Patientin gab auf Nachfrage an, sie sei wegen anhaltender Knieschmerzen vorübergehend mit einem Benzodiazepin behandelt worden. Anhand der Urinkontrollen und der Patientenangaben gibt es somit keine Hinweise auf den Gebrauch von Schlafmedikamenten während der Therapie. 


\subsection{Subjektive Schlafparameter vor und nach der Behandlung}

\subsubsection{Die subjektive Bewertung des Schlafes im Rückblick auf die vergangenen zwei Wochen (SF-B)}

Mit dem Fragebogen SF-B wurden Angaben der Patienten zu quantitativen und qualitativen Parametern ermittelt. Die Patienten füllten während ihres Aufenthaltes im Schlaflabor vor und nach der Behandlung den Schlaffragebogen SF-B (s. Kapitel 2.3.3) aus, der sich auf die zwei den Polysomnografienächten vorhergehenden Wochen bezieht. Es gibt demnach zwei Messzeitpunkte: die Zeitpunkte der Eingangs- (EP) und der Kontrollpolysomnografien (KP).

\subsubsection{SF-B - quantitative Parameter}

Die quantitativen Aspekte des subjektiven Schlafes der vergangenen zwei Wochen zeigen sich ausschließlich in Daten aus dem SF-B über folgende Parameter: tatsächliche Schlafzeit (TST), nächtliche Wachzeit (WASO), Einschlaflatenz (EL) und Häufigkeit der Schlafunterbrechungen (HdSU).

tatsächliche Schlafzeit TST (SF-B)

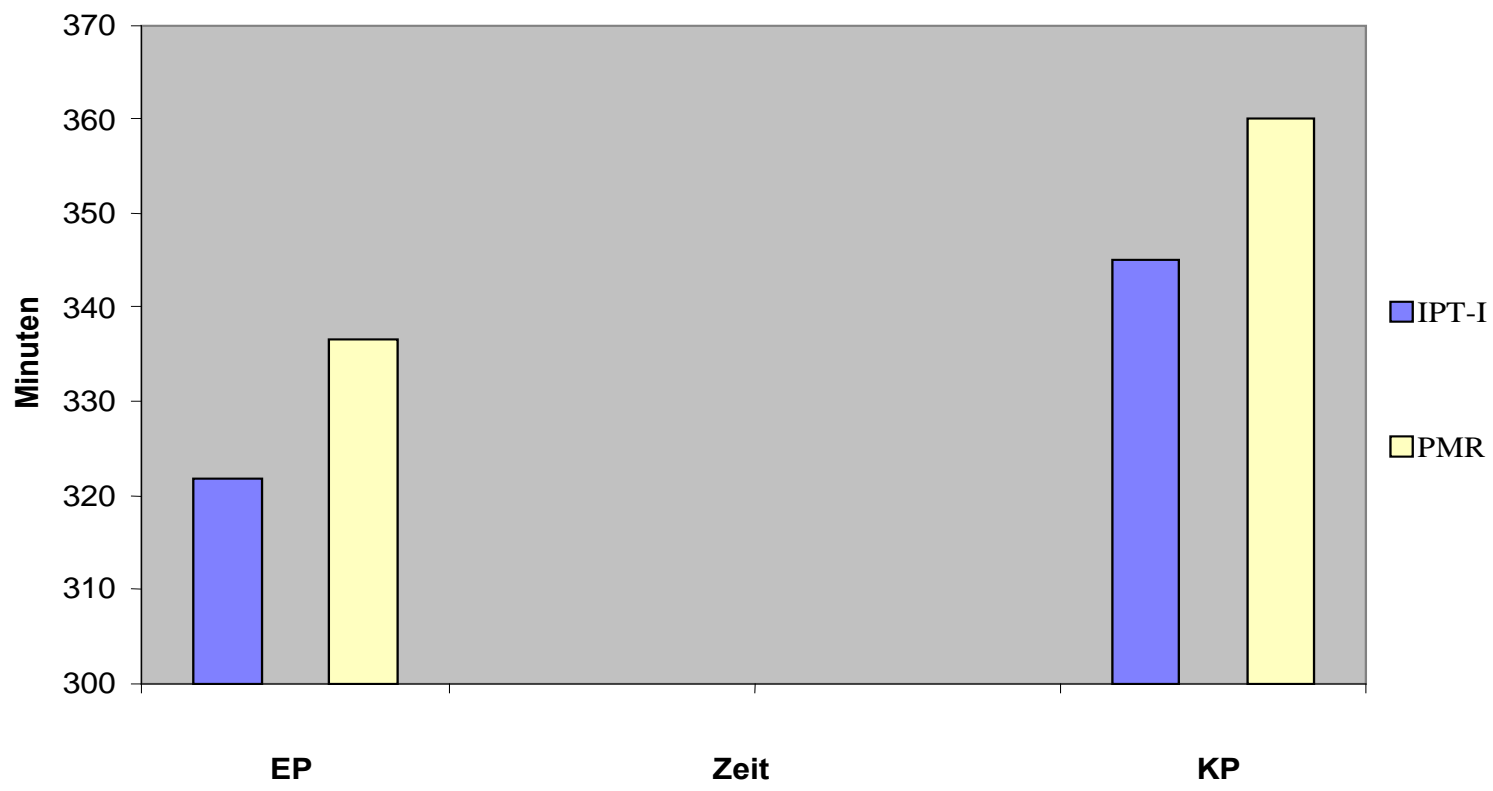

Abb. 3.01: SF-B-Schlafparameter: TST, arithmetische Mittelwerte zum Zeitpunkt der Eingangs- (EP) und Kontrollpolysomnografie (KP). 
Die subjektive tatsächliche Schlafzeit (TST) verlängerte sich nur insignifikant (t-Tests s.u.) während der Therapie: bei der IPT-I-Gruppe von durchschnittlich 322 min auf 345 min, bei der PMR-Gruppe von 337 min auf 360 min.

\section{nächtliche Wachzeit WASO (SF-B)}

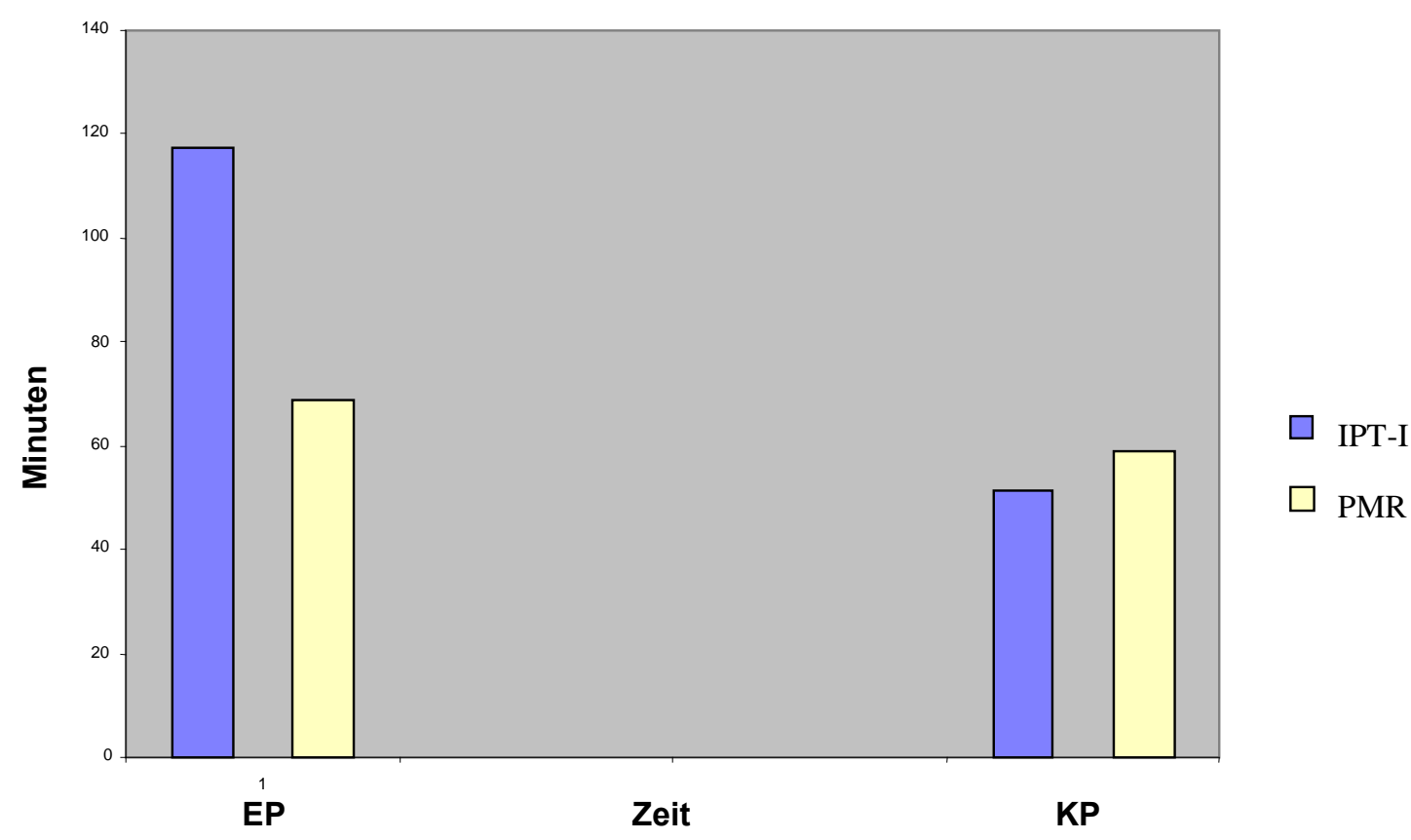

Abb. 3.02: SF-B-Schlafparameter: WASO, arithmetische Mittelwerte zum Zeitpunkt der Eingangs- (EP) und Kontrollpolysomnografie (KP).

Die subjektive nächtliche Wachzeit (WASO) reduzierte sich bei beiden Gruppen signifikant (t-Test s.u.): bei der IPT-I-Gruppe von 117 min auf 51 min, damit um mehr als die Hälfte. Die PMR-Patienten verminderten ihre anfänglich niedrigere Wachzeit ebenfalls signifikant von durchschnittlich 69 min auf 59 min.

Ob die subjektive Schlafquantität sich nach der Behandlung verbesserte, das heißt ob die Patienten länger und mit weniger Unterbrechung schliefen als vorher, konnte sich sowohl in der tatsächlichen Schlafzeit (TST) als auch in der Gesamtzeit der Schlafunterbrechungen (WASO) niederschlagen. Deshalb wurden diese Parameter in einer multivariaten Varianzanalyse überprüft und zusammen berechnet (Tab. 3.01). 
Multivariate Varianzanalyse TST und WASO (SF-B)

\begin{tabular}{|c|c|c|c|c|c|}
\hline Varianzquelle & Wert & Hyp. df & Fehl. df & F & Sign. \\
\hline Gruppe & 0,04111 & 2,00 & 22,00 & 0,47161 & 0,630 \\
\hline Zeit & 0,39792 & 2,00 & 22,00 & $\mathbf{7 , 2 6 9 9 4}$ & $\mathbf{0 , 0 0 4}$ \\
\hline Gruppe x Zeit & 0,21669 & 2,00 & 22,00 & 3,04291 & 0,068 \\
\hline
\end{tabular}

Tab. 3.01: Multivariate Varianzanalyse über tatsächliche Schlafzeit TST und nächtliche Wachzeit WASO des $S F-B$

Im SF-B liegt in der subjektiven Schlafquantität zwischen den Gruppen IPT-I und PMR kein signifikanter Unterschied vor, jedoch gibt es über die Zeit bei beiden Gruppen signifikante Unterschiede. Ein Interaktionseffekt liegt tendenziell vor, er beruht auf der in der IPT-Gruppe zu Behandlungsbeginn hohen nächtlichen Wachzeit, die sich um mehr als die Hälfte verkürzt.

Die Veränderungen wurden in einem zweiten Auswertungsschritt für jede Gruppe und jede Variable getrennt mit zweiseitigen verbundenen t-Tests untersucht: (1. TST: IPT-I: $\mathrm{p}=0,428$; PMR: $\mathrm{p}=0,111 ; 2$. WASO: IPT-I: $\mathrm{p}=\mathbf{0 , 0 0 3 4 3}$; PMR: $\mathrm{p}=\mathbf{0 , 0 0 0}$ ).

Die Daten des SF-B zu den weiteren quantitativen Parametern Einschlaflatenz (EL) und Häufigkeit der Schlafunterbrechungen (HdSU) liegen auf Ordinaldatenniveau vor: Den Patienten standen für die Einschlaflatenz sechs Antwortmöglichkeiten und für die Häufigkeit der Schlafunterbrechungen fünf Antwortmöglichkeiten zur Verfügung. Die beiden folgenden Tabellen (Tab. 3.02, Tab. 3.03) beschreiben die Lage der Mediane.

\begin{tabular}{|l|l|l|}
\hline Einschlaflatenz & \multicolumn{1}{|c|}{ EP } & \\
EL (SF-B) & & \\
Lage der Mediane & 3 & 3 \\
\hline IPT-I & 3 & 3 \\
\hline PMR & 4 & \\
\hline
\end{tabular}

Tab. 3.02: SF-B-Schlafparameter: Einschlaflatenz EL der vergangenen zwei Wochen, Mediane zum Zeitpunkt der Eingangs- (EP) und Kontrollpolysomnografie (KP). Zur Höhe der Einschlaflatenz gibt es sechs Antwortmöglichkeiten: „, weniger als 5 Min. “ (1), „5 bis 10 Min.“ (2), „10 bis 20 Min.“ (3), „20 bis 30 Min.“ (4), „,30 Min. bis 1 Stunde“ (5), „mehr als 1 Stunde“ (6). Eine Verbesserung der Einschlaflatenz stellt sich hier als eine Abnahme dar. 
Nach der Lage der Mediane verbessert sich die Einschlaflatenz nur in der PMR-Gruppe von Stufe 4 (20 bis 30 Min.) auf Stufe 3 (10 bis 20 Min.).

In der Einschlaflatenz unterschieden sich die Gruppen vor Therapiebeginn nicht signifikant (U-Test von Mann-Whitney, zweiseitige Testung: $z=-0,4166, p=0,6770$ ).

In einem zweiten Auswertungsschritt wurden die Veränderungen in den einzelnen Gruppen mittels zweiseitig durchgeführten Wilcoxon-Tests untersucht (IPT-I-Gruppe: $\mathrm{z}=-0,9435, \quad \mathrm{p}=0,3454 ;$ PMR-Gruppe: $\mathrm{z}=-2,2672, \quad \mathrm{p}=\mathbf{0 , 0 2 3 4}$ ). Die Abnahme der Einschlaflatenz ist nur in der PMR-Gruppe signifikant.

\begin{tabular}{|l|l|l|}
\hline $\begin{array}{l}\text { Häufigkeit der } \\
\text { SchlafunterbrechungenHdSU } \\
\text { (SF-B) }\end{array}$ & EP & KP \\
Lage der Mediane & 4 & 4 \\
\hline IPT-I & 4 & 4 \\
\hline PMR & 4 & \\
\hline
\end{tabular}

Tab. 3.03: SF-B - Schlafparameter: Häufigkeit der Schlafunterbrechungen HdSU der vergangenen zwei Wochen, Mediane zum Zeitpunkt der Eingangs- (EP) und Kontrollpolysomnografie (KP). Es gibt fünf Antwortmöglichkeiten zur Frage nach dem nächtlichen Wiederaufwachen: nie (1), selten (2), manchmal(3), oft (4), sehr oft(5).

In der Häufigkeit der Schlafunterbrechungen unterschieden sich die Gruppen vor der Behandlung nicht signifikant voneinander (U-Test von Mann-Whitney, zweiseitige Testung: $\mathrm{z}=-1,5165, \mathrm{p}=0,1294)$.

Beide Gruppen konnten während der Behandlung die Häufigkeit ihrer Schlafunterbrechungen nicht signifikant vermindern (IPT-I: Wilcoxon-Test, zweiseitige Testung: $z=-0,6742, p=0,5002$; PMR: Wilcoxon-Test, zweiseitige Testung: $z=-0,6668$, $\mathrm{p}=0,5049$ ).

Die vier quantitativen Parameter (TST, WASO, EL und HdSU) werden miteinander Bonferoni-adjustiert, dann gilt $\mathrm{p}=0,0125$ (s. Kapitel 2.5) und zur Beurteilung der Veränderung der subjektiven Schlafquantität der vergangenen zwei Wochen herangezogen.

Die Signifikanzen der zunächst mit den intervallskalierten Daten der Variablen TST und WASO durchgeführten Multivariaten Varianzanalysen und der mit den 
ordinalskalierten Daten der Variablen EL und HdSU durchgeführten U-Tests von Mann-Whitney sowie die Ergebnisse der anschließend mit den intervallskalierten Daten jeweils zweiseitig durchgeführten t-Tests für abhängige Stichproben und mit den ordinalskalierten Daten jeweils zweiseitig durchgeführten Wilcoxon-Tests sind der folgenden Tabelle zu entnehmen.

Übersicht Schlafquantität ( SF-B )

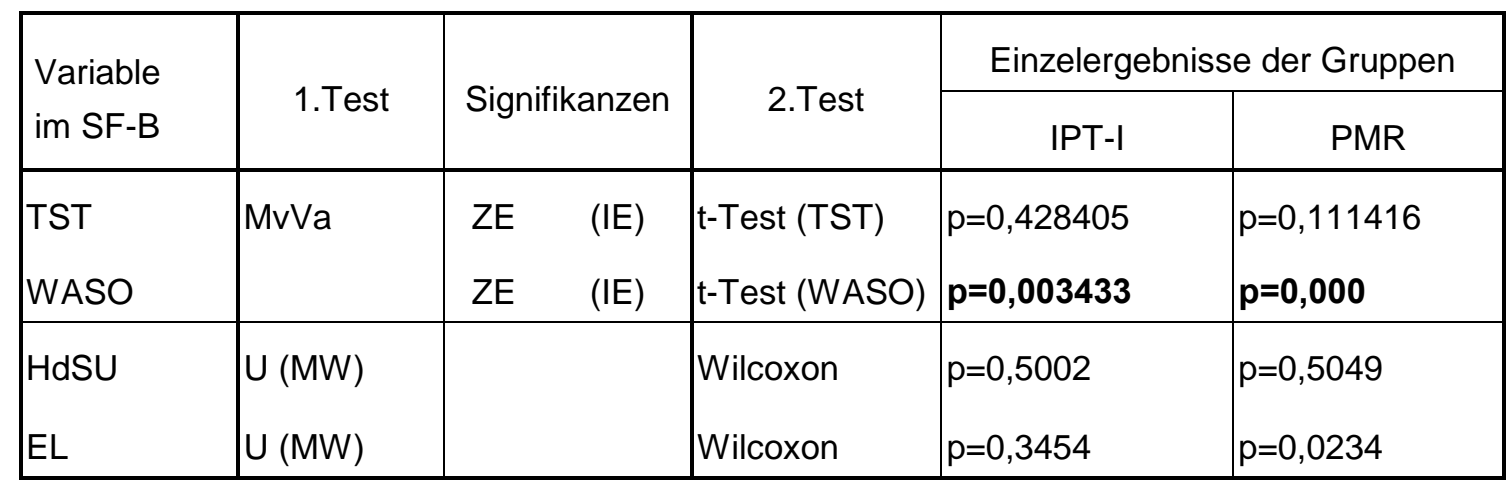

Tab. 3.04: SF-B - subjektive Schlafquantität der vergangenen zwei Wochen, quantitative Parameter: TST = tatsächliche Schlafzeit, WASO = nächtliche Wachzeit, HdSU = Häufigkeit der Schlafunterbrechungen, $E L=$ Einschlaflatenz. $M v V a=$ Multivariate Varianzanalyse, $Z E=$ Zeiteffekt, (IE) $=$ knapp verfehlter Interaktionseffekt.

Im SF-B waren die Veränderungen der tatsächlichen Schlafzeit und der Häufigkeit der Schlafunterbrechungen in keiner Gruppe signifikant. Die Gesamtzeit der Schlafunterbrechungen verkürzte sich dagegen in beiden Gruppen signifikant. Eine signifikante Minderung der subjektiven Einschlaflatenz konnte für die IPT-IGruppe nicht nachgewiesen werden. Die PMR-Gruppe schlief dagegen nach ihrer Einschätzung binnen signifikant kürzerer Zeit ein, diese Signifikanz geht jedoch durch die Adjustierung verloren.

Für beide Gruppen gibt es jeweils nur eine signifikante Abnahme der nächtlichen Gesamtwachzeit.

\subsubsection{SF-B - qualitative Parameter}

Die qualitativen Aspekte des subjektiven Schlafes der vergangenen zwei Wochen zeigen sich in den Parametern „Schlafqualität“ (SQ) und „morgendliches Erholungsgefühl“ $(\mathrm{mE})$.

Schlafqualität (SQ) und morgendliches Erholungsgefühl (mE) wurden mit getrennten univariaten Varianzanalysen berechnet. 


\section{Schlafqualität SQ (SF-B)}

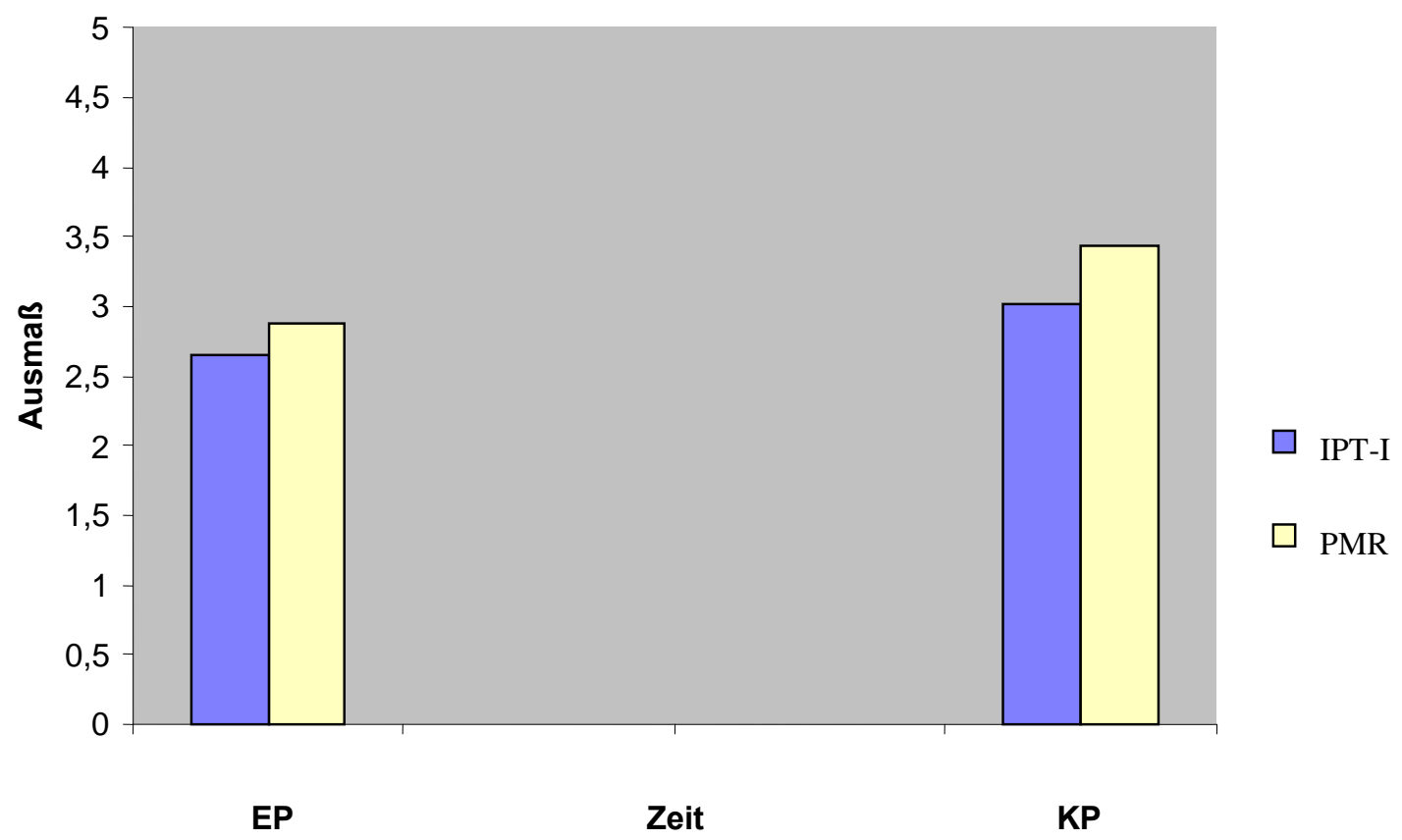

Abb. 3.03: SF-B-Schlafparameter: SQ, arithmetische Mittelwerte zum Zeitpunkt der Eingangs- (EP) und Kontrollpolysomnografie (KP)

Im Laufe der Behandlung veränderte sich die Bewertung der Schlafqualität im SF-B. Die Mittelwerte lagen zum Zeitpunkt der Eingangspolysomnografie in der IPT-I-Gruppe bei 2,66 und stiegen insignifikant auf 3,01 zum Zeitpunkt der Kontrollpolysomnografie (t-Test s.u.). Die PMR-Gruppe verbesserte sich signifikant von 2,87 auf 3,44 (t-Test s.u.). Der mögliche Maximalwert betrug 5,00. 
Univariate Varianzanalyse SQ ( SF-B)

\begin{tabular}{|c|c|c|c|c|c|}
\hline Varianzquelle & SS & df & MS & F & Sign. \\
\hline zwischen den Vpn & 6,07 & 23 & 0,26 & & \\
\hline Gruppe & 1,30 & 1 & 1,30 & $\mathbf{4 , 9 4}$ & $\mathbf{0 , 0 3 6}$ \\
\hline Zeit & 2,64 & 1 & 2,64 & $\mathbf{1 1 , 3 1}$ & $\mathbf{0 , 0 0 3}$ \\
\hline Gruppe x Zeit & 0,14 & 1 & 0,14 & 0,62 & 0,439 \\
\hline
\end{tabular}

Tab. 3.05: Univariate Varianzanalyse über die Schlafqualität SQ des SF-B. Vpn = Versuchspersonen.

Es sind ein signifikanter Gruppeneffekt und ein signifikanter Zeiteffekt nachweisbar, jedoch kein Interaktionseffekt. Die IPT-Gruppe unterscheidet sich demnach schon vor der Behandlung von der PMR-Gruppe durch die subjektiv schlechtere Schlafqualität.

Anschließend wurden zweiseitige t-Tests für abhängige Stichproben durchgeführt: IPTI-Gruppe: $p=0,17898$; PMR-Gruppe: $\mathbf{p = 0 , 0 0 0 6 1}$. Die Verbesserung der Schlafqualität war nur in der PMR-Gruppe signifikant.

Analog zur Schlafqualität wurde das Gefühl des Erholtseins nach dem Schlaf beurteilt und errechnet, das im Folgenden als morgendliches Erholungsgefühl (mE) referiert wird. 


\section{morgendliches Erholungsgefühl mE (SF-B)}

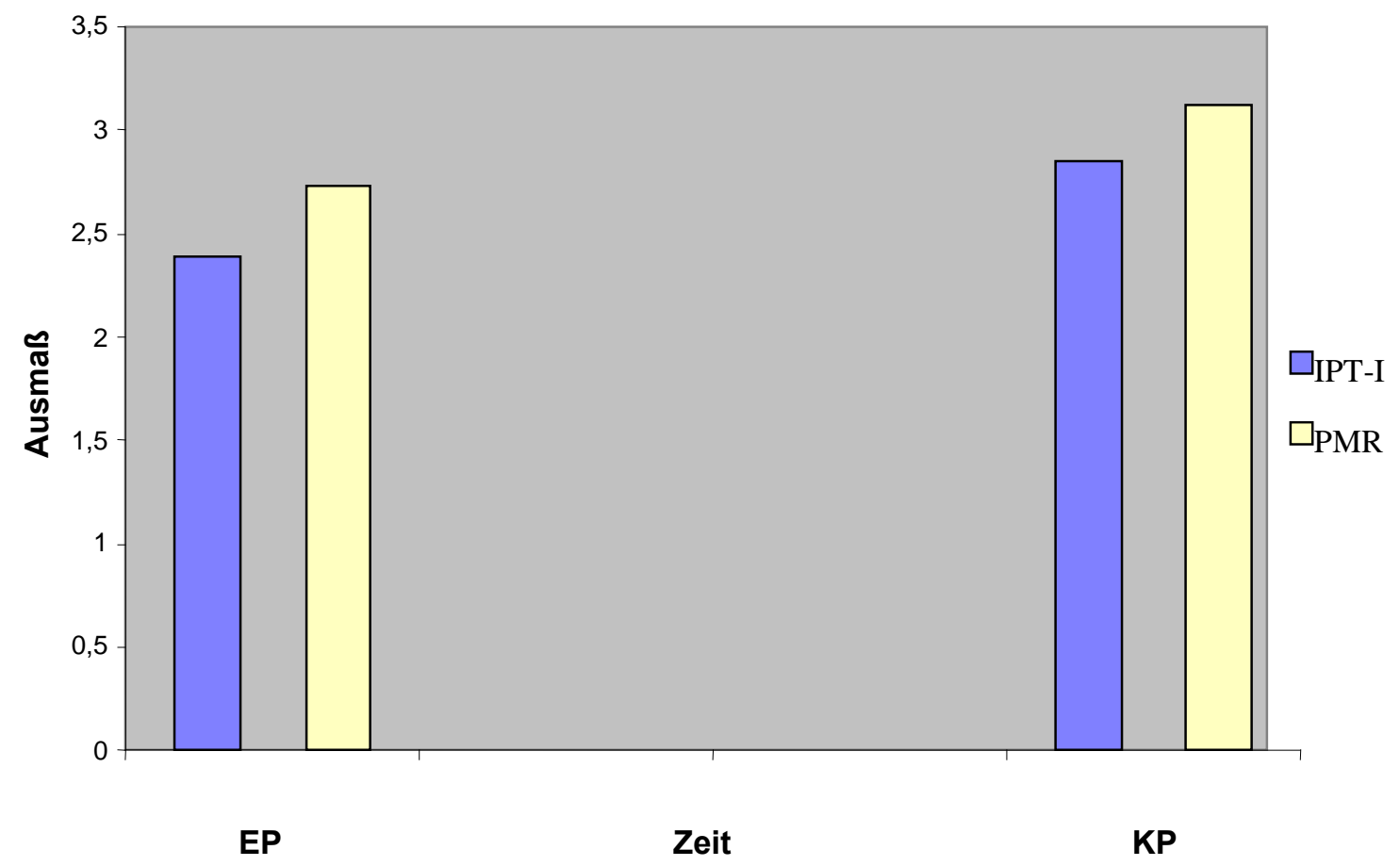

Abb. 3.04: SF-B-Schlafparameter: $m E$, arithmetische Mittelwerte zum Zeitpunkt der Eingangs- (EP) und Kontrollpolysomnografie (KP)

Die Mittelwerte des morgendlichen Erholungsgefühls verbesserten sich in Richtung auf das Maximum von 5,0 in der IPT-I-Gruppe nur insignifikant von 2,39 auf 2,85 (t-Test s.u.). In der PMR-Gruppe stieg das morgendliche Erholungsgefühl dagegen signifikant von 2,74 auf 3,12 an (t-Test s.u.). 
Univariate Varianzanalyse mE ( SF-B )

\begin{tabular}{|c|c|c|c|c|c|}
\hline Varianzquelle & SS & df & MS & F & Sign. \\
\hline zwischen den Vpn & 18,49 & 23 & 0,80 & & \\
\hline Gruppe & 2,21 & 1 & 2,21 & 2,75 & 0,111 \\
\hline Zeit & 1,19 & 1 & 1,19 & 3,69 & 0,067 \\
\hline Gruppe x Zeit & 0,05 & 1 & 0,05 & 0,17 & 0,687 \\
\hline
\end{tabular}

Tab.3.06: Univariate Varianzanalyse über das morgendliche Erholungsgefühl mE des SF-B. Vpn= Versuchspersonen.

Die Gruppen unterscheiden sich vor Behandlungsbeginn nicht. Ein Zeiteffekt verfehlt nur knapp die Signifikanz, ein Interaktionseffekt ist nicht nachweisbar.

Anschließend wurden zweiseitige t-Tests für abhängige Stichproben durchgeführt: IPTI-Gruppe: p=0,1831; PMR-Gruppe: $\mathbf{p = 0 , 0 2 3 7 4 . ~}$

Die zwei Einzeltests des SF-B (s. Kapitel 2.3.4) über SQ und mE werden miteinander Bonferoni-adjustiert, entsprechend einem Signifikanzniveau von p=0,025 (s. Kapitel 2.5), und zur Beurteilung der Veränderung der subjektiven Schlafqualität der zurückliegenden zwei Wochen herangezogen.

Die Daten wurden während der Aufenthalte der Patienten im Schlaflabor zu Beginn und nach Beendigung der Therapie erhoben.

Die Signifikanzen der zunächst mit den Daten der intervallskalierten Variablen SQ und mE durchgeführten Univariaten Varianzanalysen und die Ergebnisse der anschließend durchgeführten zweiseitigen t-Tests für abhängige Stichproben sind der folgenden Tabelle zu entnehmen. 
Übersicht Schlafqualität ( SF-B )

\begin{tabular}{|l|l|c|c|c|c|}
\hline \multirow{2}{*}{ Variable } & \multirow{2}{*}{ 1.Test } & \multirow{2}{*}{ Signifikanzen } & 2.Test & \multicolumn{2}{|c|}{ Einzelergebnisse der Gruppen } \\
\cline { 5 - 6 } & & & & IPT-I & PMR \\
\hline SQ SF-B & UvVa & GE ZE & t-Test(gepaart) & $\mathbf{p}=0,17898$ & $\mathbf{p}=\mathbf{0 , 0 0 0 6 1}$ \\
mE SF-B & UvVa & (ZE) & t-Test(gepaart) & $P=0,1831$ & $\mathbf{p = 0 , 0 2 3 7 4}$ \\
\hline
\end{tabular}

Tab. 3.07: SF-B - subjektive Schlafqualität der vergangenen zwei Wochen, qualitative Parameter: $S Q=$ Schlafqualität, $m E=$ morgendliches Erholungsgefühl. UvVa= Univariate Varianzanalyse, $G E=$ Gruppeneffekt, $Z E=$ Zeiteffekt, $(\mathrm{ZE})=$ knapp verfehlter Zeiteffekt.

Im SF-B bestätigte sich die Signifikanz der Verbesserung der Schlafqualität bei Betrachtung der einzelnen Gruppen nur für die PMR-Gruppe (zweiseitiger t-Test für abhängige Stichproben), für die IPT-I-Gruppe dagegen nicht, für sie bessert sich das morgendliche Erholungsgefühl nur tendenziell. Die Signifikanz des morgendlichen Erholungsgefühls im SF-B der PMR-Gruppe hält einer Adjustierung stand, hat jedoch in der Varianzanalyse den Zeiteffekt knapp verfehlt.

Vor Beginn der Behandlung ist die Schlafqualität der IPT-I Gruppe signifikant schlechter als die der PMR-Gruppe. Die Vergleichbarkeit beider Gruppen vor Behandlungsbeginn hinsichtlich der subjektiven Schlafqualität ist aufgrund des Gruppeneffektes nicht gegeben.

\subsubsection{Die subjektive Bewertung des Schlafes im Rückblick auf die vergangene Nacht (SF-A)}

Mit dem Schlaffragebogen A (SF-A, s. Kapitel 2.3.3) werden ebenfalls Angaben der Patienten zu quantitativen und qualitativen Parametern ermittelt. Der SF-A stimmt inhaltlich bis auf den zeitlichen Bezugsrahmen fast vollständig mit dem SF-B überein und wurde analog ausgewertet.

Die Patienten füllten während des Aufenthaltes im Schlaflabor den SF-A aus, der sich auf die zwei einzelnen Ableitungsnächte zu den Zeitpunkten EP und KP vor und nach der Therapie bezieht.

\subsubsection{SF-A - quantitative Parameter}

Die quantitativen Aspekte des subjektiven Schlafes der jeweils vergangenen Nacht zeigen sich anhand der folgenden Parameter: tatsächliche Schlafzeit (TST), Gesamtzeit der Schlafunterbrechungen (WASO), Einschlaflatenz (EL) und Häufigkeit der Schlafunterbrechungen (HdSU). 
tatsächliche Schlafzeit TST (SF-A)

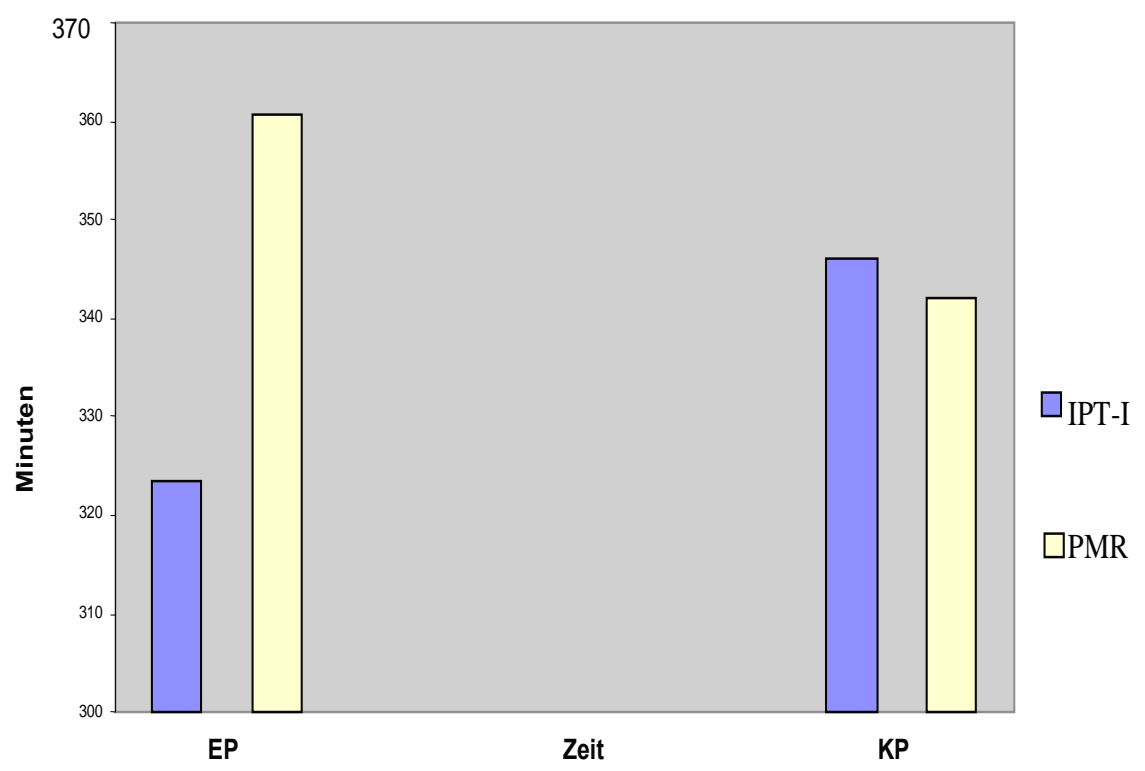

Abb. 3.05: SF-A-Schlafparameter: TST, arithmetische Mittelwerte zu den Zeitpunkten der Eingangs- (EP) und Kontrollpolysomnografie (KP)

Die tatsächliche Schlafzeit TST zeigt in der IPT-I-Gruppe eine Verbesserung, in der PMR-Gruppe dagegen eine Verschlechterung, diese Veränderungen sind jedoch nicht signifikant (s.Tab. 3.08). Die IPT-I-Gruppe kann ihre tatsächliche Schlafzeit von 323 auf 346 Minuten steigern, in der PMR-Gruppe nimmt sie dagegen von 361 auf 342 Minuten ab. 


\section{nächtliche Wachzeit WASO (SF-A)}

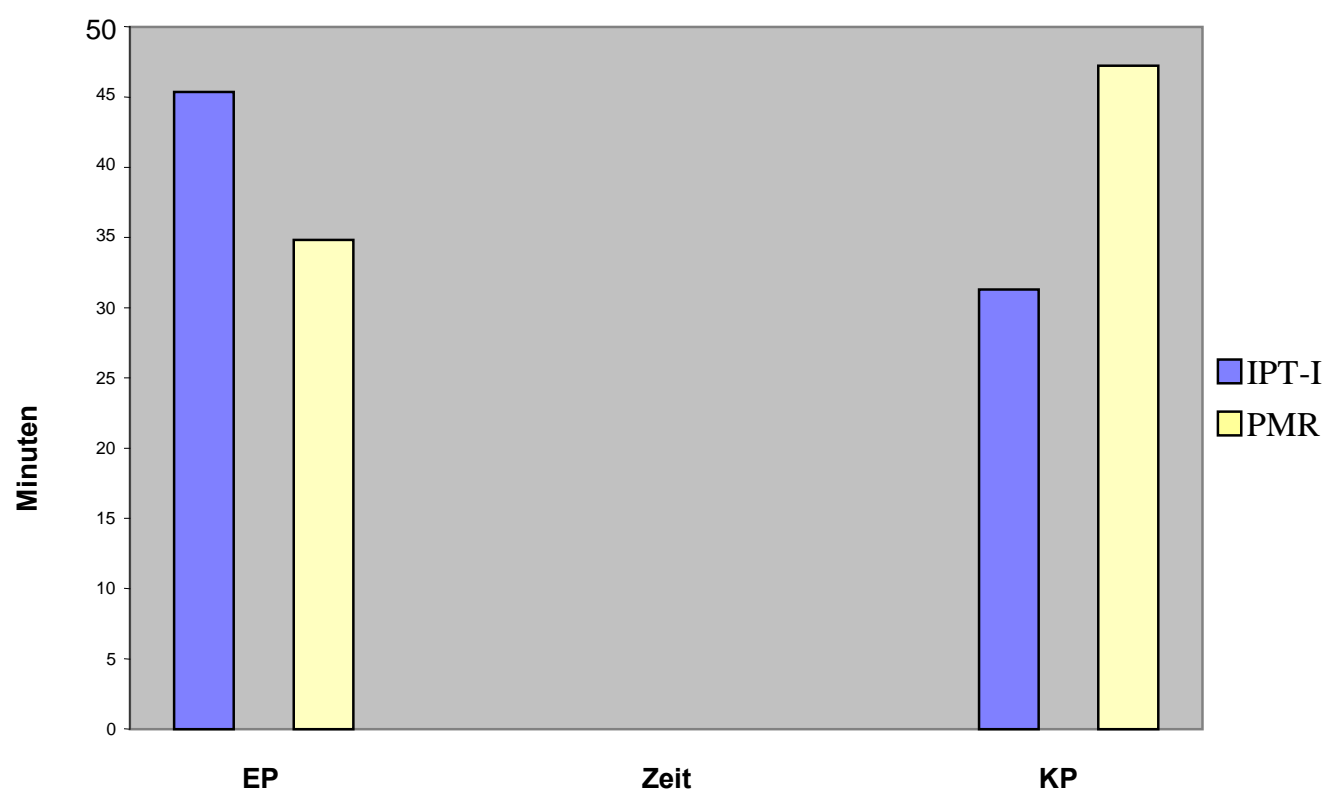

Abb. 3.06: SF-A-Schlafparameter: WASO, arithmetische Mittelwerte zu den Zeitpunkten der Eingangs(EP) und Kontrollpolysomnografie (KP)

Die Wachzeit WASO verändert sich in den Gruppen gegensätzlich: In der IPT-I-Gruppe nimmt sie ab von 45 auf 31 Minuten, in der PMR-Gruppe nimmt sie zu von 35 auf 47 Minuten. Diese Veränderungen sind jedoch ebenfalls nicht signifikant.

Beide Parameter (TST, WASO) wurden in einer multivariaten Varianzanalyse berechnet, da es sich um Intervalldaten handelt.

Multivariate Varianzanalyse TST und WASO ( SF-A)

\begin{tabular}{|c|c|c|c|c|c|}
\hline Varianzquelle & Wert & Hyp. df & Fehl. df & F & Sign. \\
\hline Gruppe & 0,9359 & 2,00 & 22,00 & 1,13582 & 0,339 \\
\hline Zeit & 0,05887 & 2,00 & 22,00 & 0,68802 & 0,513 \\
\hline Gruppe x Zeit & 0,10617 & 2,00 & 22,00 & 1,30654 & 0,291 \\
\hline
\end{tabular}

Tab. 3.08: Multivariate Varianzanalyse über nächtliche Wachzeit WASO und tatsächliche Schlafzeit TST des SF-A 
Der Tabelle 3.08 ist zu entnehmen, dass weder signifikante Unterschiede zwischen den Gruppen noch Veränderungen über die Zeit vorliegen, auch ein Interaktionseffekt ist nicht nachweisbar.

Die Daten des SF-A zu den weiteren quantitativen Parametern Einschlaflatenz (EL) und Häufigkeit der Schlafunterbrechungen (HdSU) liegen auf Ordinaldatenniveau vor: Den Patienten standen wie im SF-B für die Einschlaflatenz sechs Antwortmöglichkeiten und für die Häufigkeit der Schlafunterbrechungen fünf Antwortmöglichkeiten zur Verfügung. Die beiden folgenden Tabellen (Tab. 3.09, Tab. 3.10) beschreiben die Lage der Mediane.

\section{Einschlaflatenz (SF-A)}

\begin{tabular}{|l|l|l|}
\hline Einschlaflatenz & \multicolumn{1}{|c|}{ EP } & \\
EL (SF-A) & & \\
\hline Lage der Mediane & 4 & 3 \\
\hline IPT-I & 3 & 4 \\
\hline PMR & & \\
\hline
\end{tabular}

Tab. 3.09: SF-A-Schlafparameter: Einschlaflatenz EL, Mediane zum Zeitpunkt der Eingangs- (EP) und Kontrollpolysomnografie (KP). Zur Höhe der Einschlaflatenz gibt es sechs Antwortmöglichkeiten: „, weniger als 5 Min.“ (1), „5 bis 10 Min.“ (2), „10 bis 20 Min.“ (3), „20 bis 30 Min.“ (4), „30 Min. bis 1 Stunde“ (5), „mehr als 1 Stunde“ (6). Eine Verbesserung der Einschlaflatenz stellt sich hier als eine Abnahme dar.

Die Veränderung der EL ist gegensätzlich: Die IPT-I-Gruppe zeigt eine signifikante Verringerung, die PMR-Gruppe eine tendenzielle Zunahme (s.u.).

Vor der Therapie unterschieden sich die beiden Gruppen nicht signifikant voneinander (U-Test von Mann-Whitney, zweiseitige Testung: $z=-0,9574, p=0,3384$ ).

Die Auswertung für den Vergleich zu den Zeitpunkten EP und KP erfolgte mittels Wilcoxon Test, zweiseitige Testung: IPT-I-Gruppe: $z=-2,3117, \mathbf{p}=\mathbf{0 , 0 2 0 8}$; PMRGruppe: $\mathrm{z}=-0,5331, \mathrm{p}=0,5940$.

Die Daten des SF-A zur Häufigkeit der Schlafunterbrechungen (HdSU) liegen ebenfalls auf Ordinaldatenniveau vor: Den Patienten standen wie im SF-B für die Häufigkeit der 
Schlafunterbrechungen fünf Antwortmöglichkeiten zur Verfügung. Die folgende Tabelle beschreibt die Lage der Mediane.

Häufigkeit der Schlafunterbrechungen (SF-A)

\begin{tabular}{|l|l|l|}
\hline $\begin{array}{l}\text { Häufigkeit der } \\
\text { Schlafunterbrechungen } \\
\text { HdSU (SF-A) }\end{array}$ & EP & \\
Lage der Mediane & 4 & 3 \\
\hline IPT-I & 4 & 3 \\
\hline PMR & 3 & 3 \\
\hline
\end{tabular}

Tab. 3.10: SF-A - Häufigkeit der Schlafunterbrechungen HdSU der vergangenen Nacht, Mediane zum Zeitpunkt der Eingangs- (EP) und Kontrollpolysomnografie (KP). Es gibt fünf Antwortmöglichkeiten zur Frage nach dem nächtlichen Wiederaufwachen: nein (1), ja, einmal (2), ja, zweimal (3), ja, dreimal (4), mehr als dreimal (5). Eine Verbesserung stellt sich hier als eine Abnahme dar.

Die IPT-I-Gruppe wachte nach der Behandlung nachts weniger häufig auf als vorher. Die PMR-Gruppe zeigt keine Veränderung (Tab. 3.10).

Vor der Behandlung unterschieden sich die Gruppen nicht signifikant voneinander (U-Test von Mann-Whitney, zweiseitige Testung: $z=-0,3300, p=0,7414$ ).

Beide Gruppen konnten während der Behandlung die Zahl ihrer Schlafunterbrechungen nicht signifikant vermindern (IPT-I: Wilcoxon-Test, zweiseitige Testung: $z=-0,8002$, $\mathrm{p}=0,4236$; PMR: Wilcoxon-Test, zweiseitige Testung: $\mathrm{z}=-0,4446, \mathrm{p}=0,6566$ ).

Die vier Parameter TST, WASO, HdSU, EL werden miteinander Bonferoni-adjustiert (Signifikanzniveau $\mathrm{p}=0,0125$, s. Kapitel 2.5) und zur Beurteilung der subjektiven Schlafquantität im Rückblick auf die vergangene Nacht herangezogen.

Die Daten wurden während der Aufenthalte der Patienten im Schlaflabor zu Beginn und nach Beendigung der Therapie erhoben.

Die Signifikanzen der mit den intervallskalierten Daten der Variablen TST und WASO durchgeführten Multivariaten Varianzanalyse wie auch des mit den ordinalskalierten Daten der Variablen EL und HdSU durchgeführten U-Tests von Mann und Whitney und 
die Ergebnisse der anschließend durchgeführten Wilcoxon-Tests sind der folgenden Tabelle zu entnehmen.

Übersicht Schlafquantität ( SF-A )

\begin{tabular}{|l|l|l|l|l|l|}
\hline & & & & \multicolumn{2}{|c|}{ Einzelergebnisse der Gruppen } \\
\cline { 5 - 6 } $\begin{array}{l}\text { Variable } \\
\text { im SF-A }\end{array}$ & 1.Test & Signifikanzen & 2.Test & IPT-I & PMR \\
\hline TST, WASO & MvVa & & n.d. & & \\
\hline HdSU & & & n.d. & & \\
EL & & & Wilcoxon & $\mathrm{p}=0,4236$ & $\mathrm{p}=0,6566$ \\
& & & Wilcoxon & $\mathbf{p}=\mathbf{0 , 0 2 0 8}$ & $\mathrm{p}=0,5940$ \\
\hline
\end{tabular}

Tab. 3.11: SF-A-subjektive Schlafquantität in der täglichen Beurteilung, quantitative Parameter: TST = tatsächliche Schlafzeit, WASO = nächtliche Wachzeit, HdSU = Häufigkeit der Schlafunterbrechungen, $E L=$ Einschlaflatenz. $M v V a=$ Multivariate Varianzanalyse, $U(M W)=U$-Test Mann-Whitney. n.d. = 2.Test wurde mangels Signifikanz im 1. Test nicht durchgeführt.

Im SF-A ist den Daten zu WASO, TST und HdSU zu entnehmen, dass die Patienten beider Gruppen weder nach der Behandlung subjektiv länger schlafen als vorher, noch ihr nächtliches Wachliegen als kürzer einschätzen, noch die Zahl ihrer Schlafunterbrechungen signifikant vermindern können. Die IPT-I-Gruppe schätzt ihre Einschlaflatenz nach der Behandlung als signifikant geringer ein als vor Therapiebeginn, durch die Bonferoni-Adjustierung verliert sich jedoch diese Signifikanz. Für die PMR-Gruppe ist keine signifikante Minderung der subjektiven Einschlaflatenz nachweisbar.

Eine Veränderung des Schlafs in der täglichen Beurteilung kann hinsichtlich der quantitativen Parameter weder für die IPT-I- noch für die PMR-Gruppe bestätigt werden.

\subsubsection{SF-A - qualitative Parameter}

Die qualitativen Aspekte des subjektiven Schlafes der vergangenen Nacht zeigen sich in den Parametern Schlafqualität (SQ) und morgendliches Erholungsgefühl (mE). 
Schlafqualität (SQ) und morgendliches Erholungsgefühl (mE) wurden mit getrennten univariaten Varianzanalysen berechnet.

Schlafqualität SQ (SF-A)

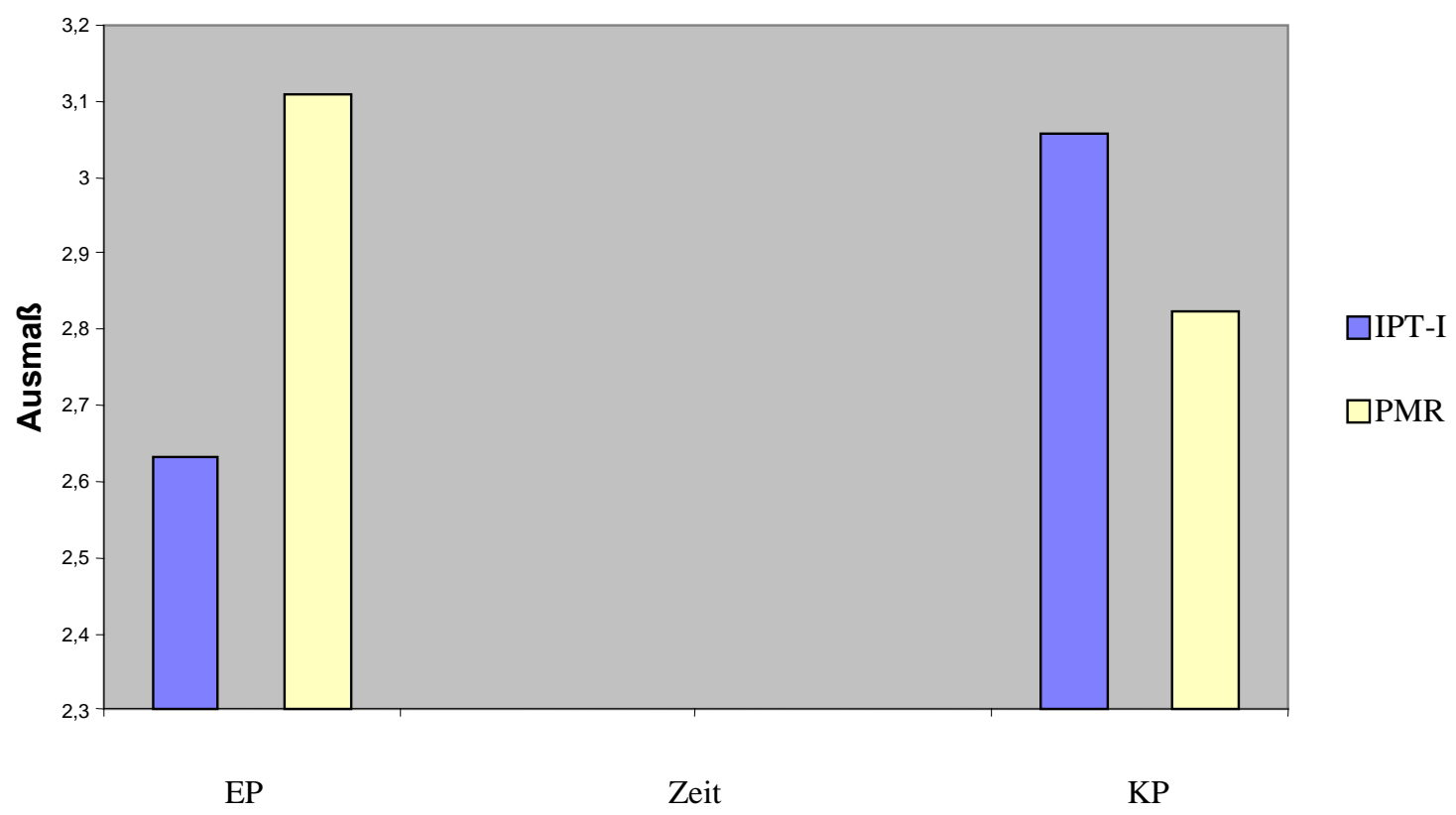

Abb. 3.07: SF-A-Schlafparameter:Schlafqualität SQ, arithmetische Mittelwerte zum Zeitpunkt der Eingangs- (EP) und Kontrollpolysomnografie (KP)

Die Schlafqualität veränderte sich im Laufe der Behandlung gegenläufig. Die Mittelwerte in der IPT-I-Gruppe stiegen fast signifikant von 2,63 auf 3,06 an, in der PMR-Gruppe verringerten sie sich dagegen insignifikant von 3,11 auf 2,82 (möglicher Maximalwert: 5,00) (t-Tests s.u.). 
Univariate Varianzanalyse SQ (SF-A)

\begin{tabular}{|c|c|c|c|c|c|}
\hline Varianzquelle & SS & df & MS & F & Sign. \\
\hline zwischen den Vpn & 11,21 & 23 & 0,49 & & \\
\hline Gruppe & 0,13 & 1 & 0,13 & 0,27 & 0,608 \\
\hline Zeit & 0,20 & 1 & 0,20 & 0,69 & 0,414 \\
\hline Gruppe x Zeit & 1,59 & 1 & 1,59 & $\mathbf{5 , 6 5}$ & $\mathbf{0 , 0 2 6}$ \\
\hline
\end{tabular}

Tab. 3.12: Univariate Varianzanalyse über die Schlafqualität SQ des SF-A

Es sind keine signifikanten Gruppen- und Zeiteffekte nachweisbar, jedoch liegt ein signifikanter Interaktionseffekt vor, d.h. die Schlafqualität veränderte sich in beiden

\section{Gruppen signifikant unterschiedlich.}

Bei der Betrachtung der Daten über die Schlafqualität des SF-A (Abb. 3.07) zeigt sich, worin der Interaktionseffekt (Tab. 3.12) besteht: Im Gegensatz zur IPT-I-Gruppe, die ihre subjektive Schlafqualität von 2,63 auf 3,06 verbessern kann, nimmt diese in der PMR-Gruppe von 3,11 auf 2,82 etwas ab. Diese Veränderungen der einzelnen Gruppen werden mittels zweiseitigen verbundenen t-Tests untersucht: IPT-I: p=0,05635; PMR: $\mathrm{p}=0,3737$. Die IPT-I-Gruppe verfehlt die Signifikanz einer Verbesserung nur knapp, die Verschlechterung der Schlafqualität in der PMR-Gruppe ist nicht signifikant.

Analog zur Schlafqualität wurde das morgendliche Erholtsein (mE) nach dem Schlaf beurteilt und varianzanalytisch berechnet. 
morgendliches Erholungsgefühl mE (SF-A)

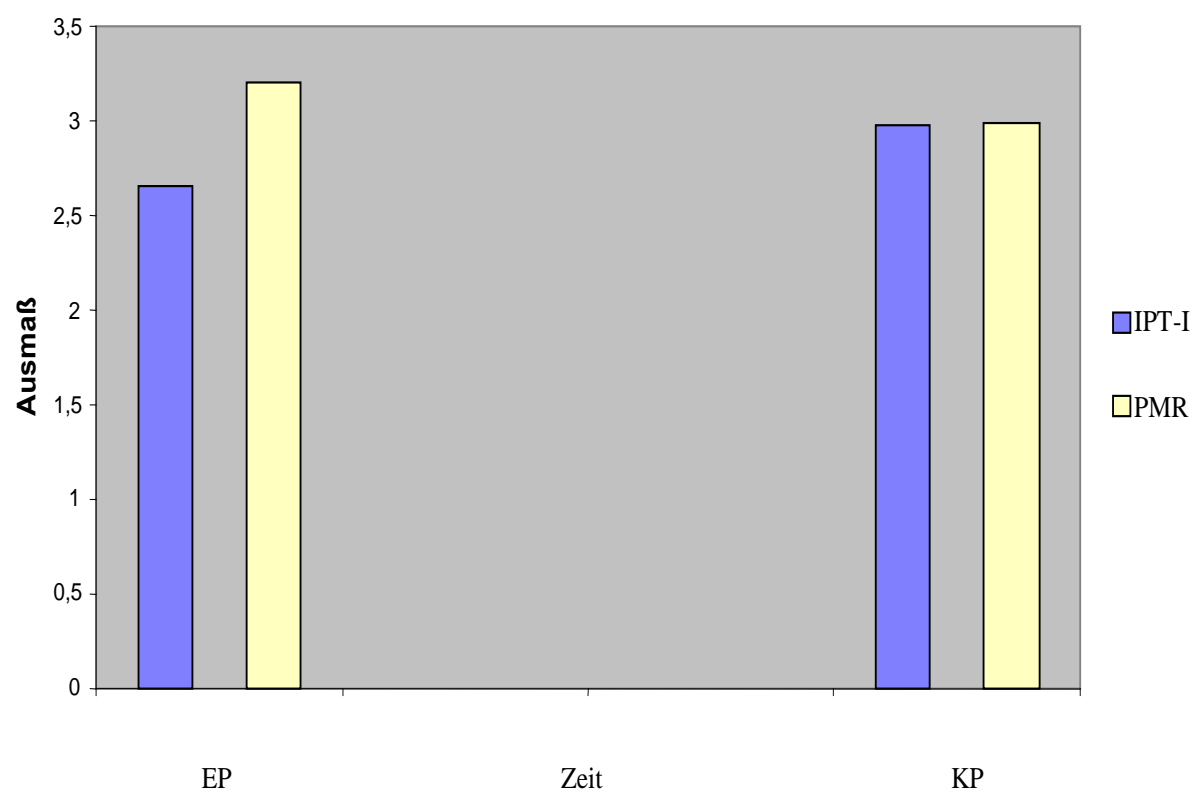

Abb. 3.08: SF-A-Schlafparameter: $m E$, arithmetische Mittelwerte der Eingangs- (EP) und Kontrollpolysomnografie (KP)

Die IPT-I-Gruppe verbessert ihr morgendliches Erholungsgefühl nur insignifikant von 2,66 auf 2,98, bei der PMR-Gruppe tritt eine insignifikante Verschlechterung von 3,21 auf 3,0 ein.

\section{Univariate Varianzanalyse mE (SF-A)}

\begin{tabular}{|c|c|c|c|c|c|}
\hline Varianzquelle & SS & df & MS & F & Sign. \\
\hline zwischen den Vpn & 20,79 & 23 & 0,90 & & \\
\hline Gruppe & 0,89 & 1 & 0,89 & 0,98 & 0,332 \\
\hline Zeit & 0,12 & 1 & 0,12 &, 50 & 0,488 \\
\hline Gruppe x Zeit & 0,65 & 1 & 0,65 & 2,76 & 0,110 \\
\hline
\end{tabular}

Tab. 3.13: Univariate Varianzanalyse über das morgendliche Erholungsgefühl mE des SF-A

Die Gruppen unterscheiden sich bezüglich des morgendlichen Erholungsgefühls nicht. Auch unter der Behandlung verändern sich die beiden Gruppen nicht signifikant, wie an den fehlenden Zeit- und Interaktionseffekten erkennbar ist (Tabelle 3.13). 
Die zwei Einzeltests des SF-A (s. Kapitel 2.3.3) über SQ und mE, die sich auf die vergangene Nacht beziehen, werden zur Beurteilung einer Veränderung des Schlafes der vergangenen Nacht Bonferoni-adjustiert (Signifikanzniveau p=0,025, s. Kapitel 2.5).

Die Daten wurden während der Aufenthalte der Patienten im Schlaflabor zu Beginn und nach Beendigung der Therapie erhoben.

Die Signifikanzen der zunächst mit den intervallskalierten Variablen SQ und mE durchgeführten Univariaten Varianzanalysen und die Ergebnisse der anschließend durchgeführten zweiseitigen t-Tests für abhängige Stichproben sind der folgenden Tabelle zu entnehmen.

Übersicht Schlafqualität ( SF-A )

\begin{tabular}{|l|l|c|c|c|c|}
\hline \multirow{2}{*}{ Variable } & \multirow{2}{*}{ 1.Test } & Signifikanzen & \multirow{2}{*}{ 2.Test } & \multicolumn{2}{|c|}{ Einzelergebnisse der Gruppen } \\
\cline { 5 - 6 } & & & & IPT-I & PMR \\
\hline $\begin{array}{l}\text { SQ SF-A } \\
\text { mE SF-A }\end{array}$ & UvVa & IE & t-Test(gepaart) & $\mathrm{p}=0,05635$ & $\mathrm{p}=0,37373$ \\
\hline
\end{tabular}

Tab. 3.14: subjektive Schlafqualität in der Beurteilung der vergangenen Nacht, qualitative Parameter: $S Q=$ Schlafqualität, $m E=$ morgendliches Erholungsgefühl. UvVa = univariate Varianzanalyse, $I E=$ Interaktionseffekt, n.d. $=2$. Test wurde mangels Signifikanz im 1. Test nicht durchgeführt.

Die IPT-I-Gruppe kann im SF-A ihre subjektive Schlafqualität nur tendenziell verbessern, bei der PMR-Gruppe verschlechtert sie sich sogar, wenn auch insignifikant. Diese unterschiedliche Veränderung findet im dargestellten Interaktionseffekt (IE) in der univariaten Varianzanalyse der Schlafqualität Ausdruck (Tab. 3.12). Im SF-A ist keine signifikante Verbesserung des morgendlichen Erholungsgefühls zu erkennen.

\subsection{Objektive Schlafparameter vor und nach der Behandlung}

Mittels Polysomnografie (PSG, s. Kapitel 2.3.2) wurden die vier Variablen Einschlaflatenz (EL), tatsächliche Schlafzeit (TST), Schlafeffizienz (SE) und nächtliches Wach (WASO) erhoben. Sie dienten der Erfassung der objektiven Schlafparameter. 
In der folgenden Tabelle 3.15 werden diese 4 Parameter zu den jeweils zwei Messzeitpunkten der Eingangspolysomnografienächte (EP) Nacht 1 und Nacht 2 und der nach Beendigung der jeweiligen Therapieverfahren abgeleiteten Kontrollpolysomnografienächte (KP) Nacht 3 und Nacht 4 gezeigt. Dargestellt sind die Mittelwert (MW) der Patientengruppen IPT-I und PMR.

Anhand der Tabelle zeigt sich, dass in den ersten beiden Nächten im Schlaflabor vor Beginn der Behandlung die IPT-I-Patienten dazu neigen, schlechter einzuschlafen, nachts länger wach zu liegen und eine geringere Schlafeffizienz zu erreichen als die PMR-Patienten.

Man sieht nach der Behandlung eine Verbesserung der Schlafeffizienz (SE) und der Einschlaflatenz (EL) in der IPT-I-Gruppe und eine Verbesserung der Parameter TST und WASO in beiden Gruppen, in der IPT-I-Gruppe auch der Einschlaflatenz.

Im Anschluss an die Tabelle folgt eine Übersichtstabelle (Tab. 3.16), in der die objektiven (PSG) zum Vergleich mit den subjektiven Schlafparametern sowohl der vergangenen Nacht (SF-A) als auch eines zurückliegenden Zweiwochenzeitraums (SFB) aufgeführt sind. 
Objektive Schlafparameter - polysomnografische Daten

Gruppenmittelwerte der Einzelnächte vor und nach der Behandlung

\begin{tabular}{|c|c|c|c|c|}
\hline & \multicolumn{2}{|c|}{ Eingangspolysomnografie } & \multicolumn{2}{|c|}{ Kontrollpolysomnografie } \\
\hline & Nacht 1 & Nacht 2 & \multirow[t]{2}{*}{ Nacht 3} & \multirow[t]{2}{*}{ Nacht 4} \\
\hline \multicolumn{3}{|c|}{ Einschlaflatenz EL } & & \\
\hline \multicolumn{5}{|l|}{ IPT-I } \\
\hline Mittelwert & 17,73 & 26,08 & 13,63 & 11,69 \\
\hline \multicolumn{5}{|l|}{ PMR } \\
\hline Mittelwert & 15,75 & 13,54 & 15 & 15,2 \\
\hline \multicolumn{5}{|c|}{ tatsächliche Schlafzeit TST } \\
\hline \multicolumn{5}{|l|}{ IPT-I } \\
\hline Mittelwert & 363,69 & 356,35 & 384,13 & 387,38 \\
\hline \multicolumn{5}{|l|}{ PMR } \\
\hline Mittelwert & 355,08 & 375,13 & 396,41 & 384,95 \\
\hline \multicolumn{5}{|c|}{ Schlafeffizienz $S E$} \\
\hline \multicolumn{5}{|l|}{ IPT-I } \\
\hline Mittelwert & 75,02 & 78,06 & 81,41 & 86,52 \\
\hline \multicolumn{5}{|l|}{ PMR } \\
\hline Mittelwert & 79,86 & 84,83 & 82,67 & 83,12 \\
\hline \multicolumn{5}{|c|}{ nächtliche Wachzeit WASO } \\
\hline \multicolumn{5}{|l|}{ IPT-I } \\
\hline Mittelwert & 91,04 & 61,50 & 66,91 & 38,92 \\
\hline \multicolumn{5}{|l|}{$P M R$} \\
\hline Mittelwert & 65,53 & 47,12 & 52,77 & 45,15 \\
\hline
\end{tabular}

Tab. 3.15: objektive Schlafparameter Einschlaflatenz EL (in Minuten=min), tatsächliche Schlafzeit TST (min), Schlafeffizienz SE (in Prozent), nächtliche Wachzeit WASO (min) zu den folgenden Messzeitpunkten: Nacht 1 und 2 der Eingangs- (=EP) und Nacht 3 und 4 der Kontrollpolysomnografie (KP). Angaben für die IPT-I- und die PMR-Gruppe jeweils mit Mittelwert. 


\section{Gegenüberstellung von objektiven und subjektiven Schlafparametern vor und} nach der Behandlung

\begin{tabular}{|c|c|c|c|c|c|c|c|c|c|}
\hline & $1 . \mathrm{N}$ & 2.N & $1 N$ & $2 \mathrm{Wo}$ & & 3.N & 4.N & $1 N$ & $2 \mathrm{Wo}$ \\
\hline $\mathbf{V}$ & $\mathbf{0}$ & $\mathbf{R}$ & & & $\mathbf{N}$ & $\mathbf{A}$ & C & $\mathbf{H}$ & \\
\hline EL & PSG & PSG & SF-A & SF-B & EL & PSG & PSG & SF-A & SF-B \\
\hline IPT-I & 17,73 & 26,08 & $20-30$ & $10-20$ & IPT-I & 13,63 & 11,69 & $10-20$ & $10-20$ \\
\hline PMR & 15,75 & 13,54 & $10-20$ & $20-30$ & PMR & 15 & 15,2 & $20-30$ & $10-20$ \\
\hline TST & PSG & PSG & SF-A & SF-B & TST & PSG & PSG & SF-A & SF-B \\
\hline IPT-I & 364 & 356 & 323 & 322 & IPT-I & 384 & 387 & 346 & 345 \\
\hline PMR & 355 & 375 & 361 & 337 & PMR & 396 & 385 & 342 & 360 \\
\hline SE/SQ & PSG & PSG & SF-A & SF-B & SE/SQ & PSG & PSG & SF-A & SF-B \\
\hline IPT-I & 75,02 & 78,06 & 2,63 & 2,66 & IPT-I & 81,41 & 86,52 & 3,06 & 3,01 \\
\hline PMR & 79,86 & 84,83 & 3,11 & 2,87 & PMR & 82,67 & 83,12 & 2,82 & 3,44 \\
\hline WASO & PSG & PSG & SF-A & SF-B & WASO & PSG & PSG & SF-A & SF-B \\
\hline IPT-I & 91,04 & 61,5 & 45 & 117 & IPT-I & 66,91 & 38,92 & 31 & 51 \\
\hline PMR & 65,53 & 47,12 & 35 & 69 & PMR & 52,77 & 45,15 & 47 & 59 \\
\hline
\end{tabular}

Tab.: 3.16: Gegenüberstellung von objektiven und subjektiven Schlafparametern vor und nach der Behandlung: Die Angaben der objektiven EL sind Mittelwerte, s. Tab. 3.15, die der subjektiven EL sind Medianklassen. $E L=$ Einschlaflatenz (in Minuten=min), TST=tatsächliche Schlafzeit (min), SE=Schlafeffizienz (in Prozent), WASO=nächtliches Wach (min). SE (objektiv) wird SQ (subjektiv) gegenübergestellt.

Die Einschlaflatenz EL wird nur nach der Behandlung von der PMR-Gruppe zu hoch eingeschätzt, die totale Schlafzeit TST wird durchweg zu niedrig eingeschätzt. 
In der Gegenüberstellung von objektiver Schlafeffizienz SE und subjektiver Schlafqualität SQ zeigt nur die IPT-I-Gruppe sowohl von der jeweils ersten zur jeweils zweiten Nacht eines Schlaflaboraufenthaltes als auch im Vorher-Nachher-Vergleich eine Verbesserung aller Mittelwerte. Die PMR-Gruppe zeigt dagegen unterschiedliche Befunde.

Die nächtliche Wachzeit WASO beider Gruppen bessert sich von der jeweils ersten zur jeweils zweiten Nacht eines Schlaflaboraufenthaltes. Sie wird in der mittelfristigen Beurteilung (SF-B) durchweg als länger bewertet als in der kurzfristigen. Nur bei diesem Parameter liefert die Einschätzung des Patienten beider Gruppen in der kurzfristigen Beurteilung überwiegend ein besseres Ergebnis als die Polysomnografie.

\subsection{Verlaufsbetrachtung subjektiver Schlafparameter (VIS-M)}

Der Gesamtverlauf des Schlafes während der Behandlungsdauer von 12 Wochen wurde an Hand der täglich morgens ausgefüllten VIS-M-Fragebögen ermittelt. In den Fragebögen bewerteten die Patienten den Schlaf der vergangenen Nacht zu Hause.

Für jede der Wochen, die den Behandlungsterminen 2 bis 12 vorhergehen, wurden fünf Nachtprotokolle der einzelnen Patienten zu einem Mittelwert zusammengefasst. Anschließend wurde aus den Mittelwerten der Patienten ein Mittelwert für die jeweilige Behandlungsgruppe gebildet.

Aus der IPT-I-Gruppe wurden für die Verlaufsbetrachtung die Daten eines Patienten eliminiert, da er Extremwerte lieferte. Dieser Patient war auch der älteste Patient der gesamten Stichprobe.

Die dahingehend bereinigten Mittelwerte der 11 Messzeiträume sind in den folgenden Abbildungen für die subjektiven Parameter tatsächliche Schlafzeit (TST), Einschlaflatenz (EL), Schlafqualität (SQ) und morgendliches Erholungsgefühl (mE) dargestellt.

Im übernächsten Unterkapitel (s. Kapitel 3.6) erfolgt, den Ergebnisteil abschließend, die eingebettete Darstellung der nun folgenden Mittelwerte der 11 Messzeiträume in die Daten der Baseline, der Eingangs- und der Kontrollpolysomnografie, die zum Teil bereits von Müller-Popkes 1997 veröffentlicht wurden. 


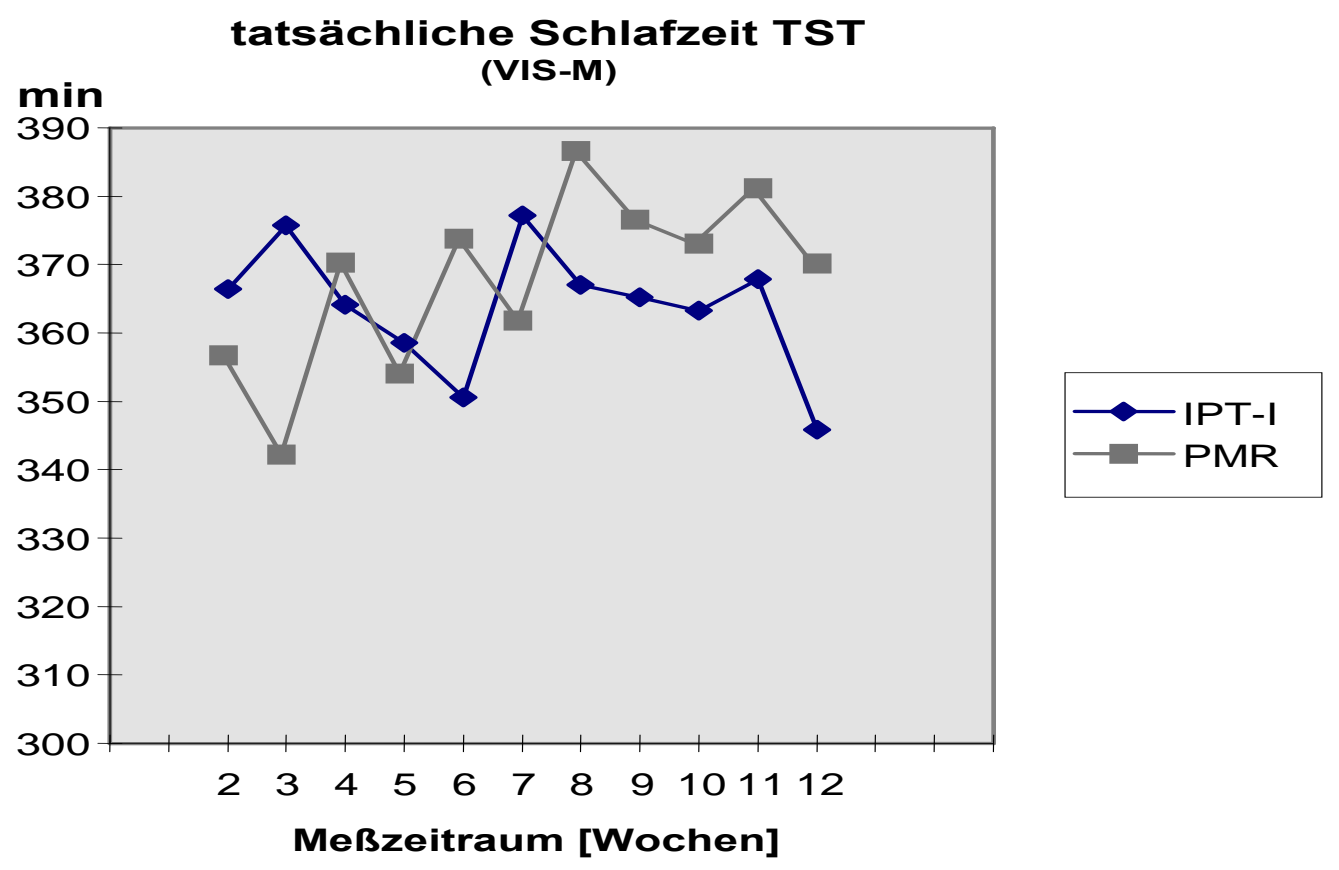

Abb. 3.09: 1. Verlaufsbetrachtung VIS-M: tatsächliche Schlafzeit (TST) in Minuten. 2-12 = wöchentliche Therapiesitzungen. Die Abszissenachse ist nicht proportional.

1. tatsächliche Schlafzeit (TST): Die PMR-Gruppe kann unter Schwankungen insgesamt einen leichten kontinuierlichen Anstieg verzeichnen und erzielt durchweg längere subjektive tatsächliche Schlafzeiten als die IPT-I-Gruppe, die im mittleren Teil der Therapie etwas einbricht. Nach der elften Sitzung ist bei beiden Gruppen ein sehr deutlicher Rückgang zu verzeichnen. Zum letzten Behandlungstermin liegt in der PMRGruppe eine diskrete Verbesserung im Vergleich zum Behandlungsbeginn vor, in der IPT-I-Gruppe dagegen eine leichte Verschlechterung. 


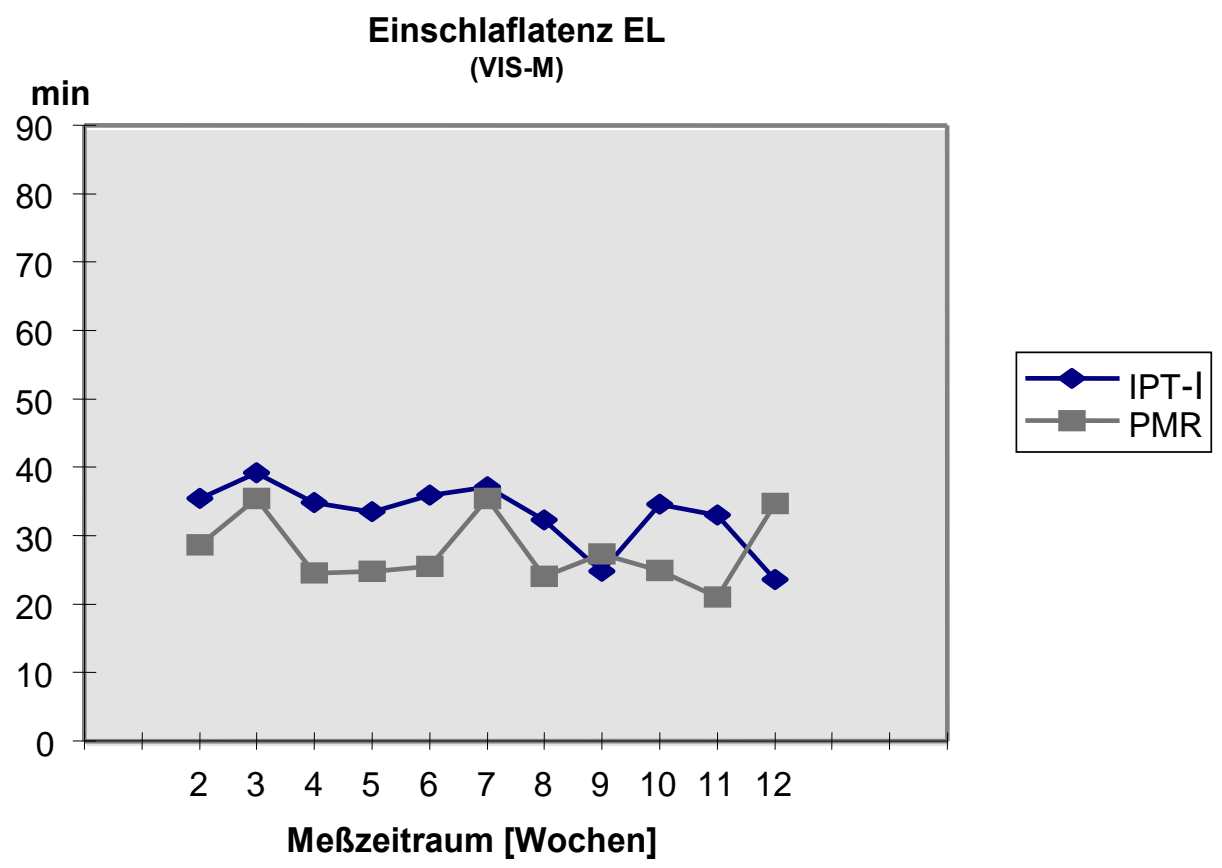

Abb. 3.10: 2. Verlaufsbetrachtung VIS-M: Einschlaflatenz (EL) in Minuten. 2-12 = wöchentliche Therapiesitzungen. Die Abszissenachse ist nicht proportional.

2. Einschlaflatenz (EL) In der IPT-I-Gruppe liegen die Werte der Einschlaflatenzen zum eigentlichen Beginn der jeweiligen Therapie (Messzeitraum 2) und während der ersten Sitzungen mit ihren Schwankungen etwas über denen der PMR-Gruppe, ab der achten Sitzung gleichen sie sich denen an und erreichen zum letzten Sitzungstermin im Vergleich zur PMR-Gruppe, die etwas ansteigt, einen besseren Wert. Die PMR-Gruppe verschlechtert sich dagegen im Laufe der Behandlung leicht. 


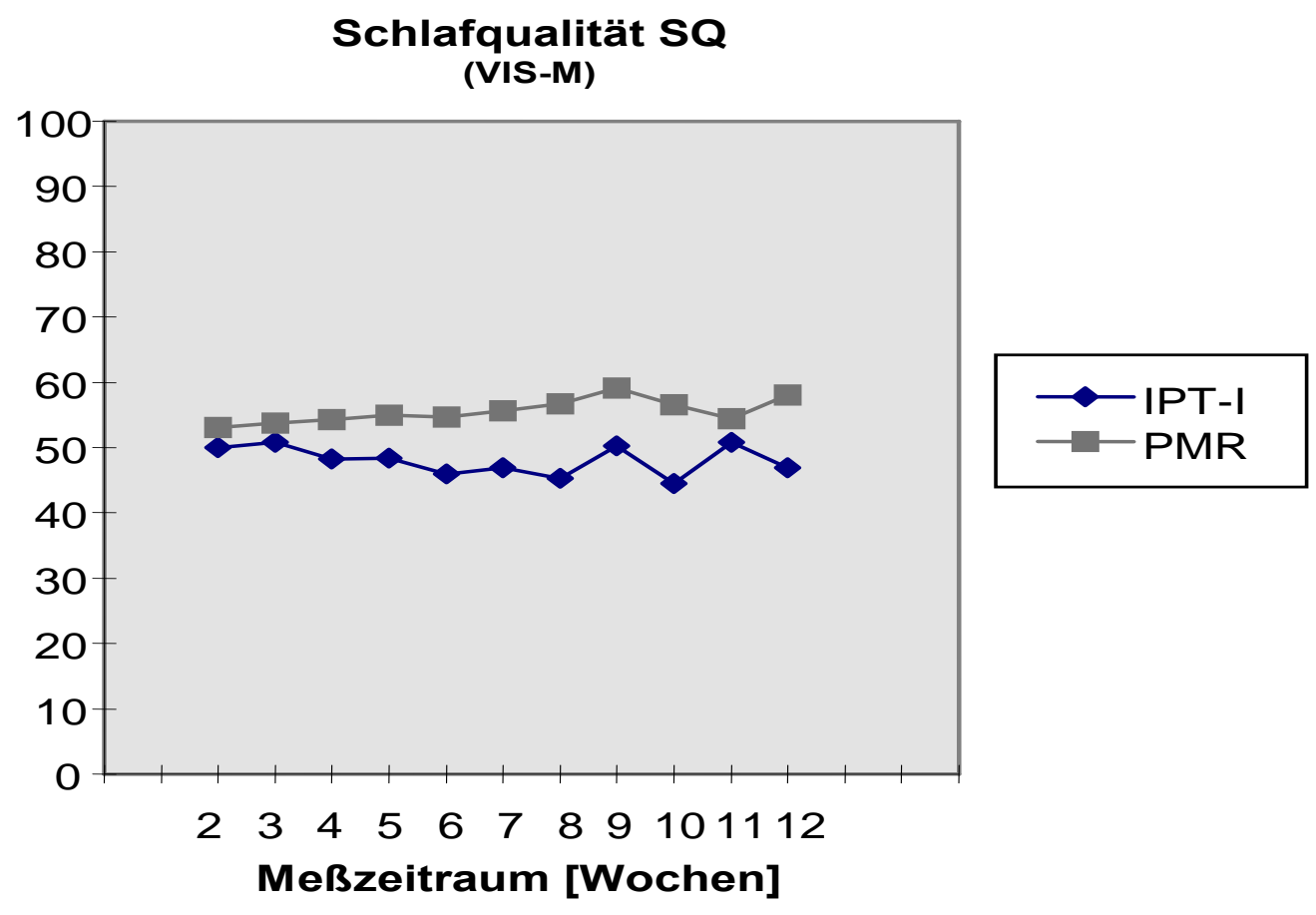

Abb. 3.11: 3. Verlaufsbetrachtung VIS-M: Schlafqualität (SQ); $0=$ sehr schlechte Nacht, $100=$ sehr gute Nacht, 2-12 = wöchentliche Therapiesitzungen. Die Abszissenachse ist nicht proportional.

3. Schlafqualität (SQ) Die subjektive Schlafqualität liegt zu Therapiebeginn bei beiden Gruppen im mittleren Bereich. Ab der dritten Sitzung verhalten sich die Gruppen gegensätzlich: Die IPT-I-Gruppe fällt unter Schwankungen leicht ab, sie zeigt zunehmende Schwankungen, die eine diskrete Besserungstendenz zum Behandlungsende hin erkennen lassen. Die PMR-Gruppe weist in ihrer zunächst kontinuierlichen mäßigen Verbesserung ab der neunten Sitzung leichte Schwankungen auf. Insgesamt nehmen die Schwankungen in beiden Gruppen im Verlauf etwas zu. Gegen Behandlungsende weichen beide Gruppen leicht auseinander, wobei sich nur die PMR-Gruppe im Vergleich zum Therapiebeginn verbessern kann. 


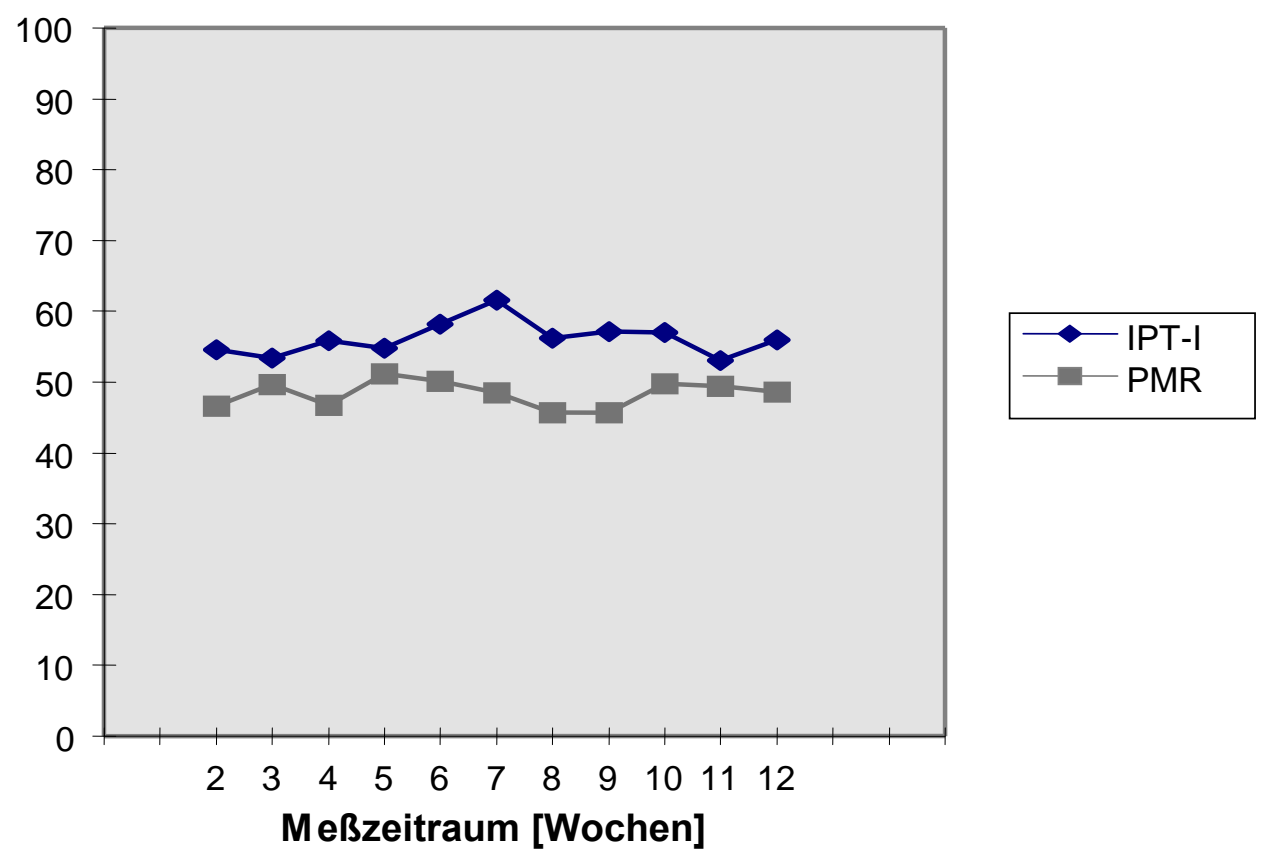

Abb. 3.12: 4. Verlaufsbetrachtung VIS-M: morgendliches Erholungsgefühl ( $\mathrm{mE}) ; 0=$ wundervoll frisch und energiegeladen, 100 = schrecklich müde und lustlos. 2-12 = wöchentliche Therapiesitzungen. Die Abszissenachse ist nicht proportional.

4. morgendliches Erholungsgefühl (mE): Zu Therapiebeginn ist das morgendliche Erholungsgefühl in der PMR-Gruppe deutlich besser als das der IPT-I-Gruppe. Nach Schwankungen im mittleren Bereich während der Behandlung ist für die PMR-Gruppe ab der elften Sitzung eine leichte Verbesserung sichtbar. Von der ersten eigentlichen Therapiesitzung bis zur letzten kommt es insgesamt zu keinen positiven Entwicklungen des durchweg im mittleren Bereich liegenden morgendlichen Erholungsgefühls. Die IPT-I-Gruppe verändert sich zunächst kaum. Zur siebten Sitzung erreicht sie die stärkste Verschlechterung ihres morgendlichen Erholungsgefühls und pendelt sich danach wieder fast auf den Wert zu Therapiebeginn ein, kann sich jedoch insgesamt nicht verbessern, durchweg ist das morgendliche Erholungsgefühl in dieser Gruppe etwas schlechter als in der PMR-Gruppe. 


\subsection{Explorative Datenanalyse des VIS-M - Veränderungen in den ersten zwei Dritteln des Behandlungszeitraums}

Um zu klären, ob sich die subjektiven Schlafparameter im Vergleich zur Baseline schon vor dem Ende der Behandlung verändern, wurden mit den intervallskalierten Daten der Parameter: tatsächliche Schlafzeit (TST), Einschlaflatenz (EL), Schlafqualität (SQ) und morgendliches Erholungsgefühl (mE) Varianzanalysen gerechnet. Drei der 14 Messzeiträume fanden Eingang in die Berechnung: 1. die Baseline (BL) (vgl. MüllerPopkes 1997), 2. die Zeit vor der zweiten Sitzung (S2), das heißt vor der ersten eigentlichen Therapiesitzung nach dem Erstgespräch und der Polysomnografie und 3. die Zeit vor der neunten Sitzung (S9). Dieser letzte Messzeitpunkt wurde zur Klärung der Frage gewählt, ob sich die Therapien auf acht Sitzungen verkürzen ließen, ohne an therapeutischer Wirksamkeit zu verlieren. Hierfür wurden wie für die Daten der vorangegangenen Verlaufsbetrachtung die Daten eines IPT-I-Patienten eliminiert, da dieser Extremwerte lieferte. Er war der älteste Patient der Stichprobe.

Multivariate Varianzanalyse TST und EL (VIS-M, 3 Messzeitpunkte)

\begin{tabular}{|c|c|c|c|c|c|}
\hline Varianzquelle & Wert & Hyp. df & Fehl. df & F & Sign. \\
\hline Gruppe & 0,00750 & 2,00 & 21,00 & 0,07933 & 0,924 \\
\hline Zeit & 0,46312 & 4,00 & 19,00 & $\mathbf{4 , 0 9 7 3 3}$ & $\mathbf{0 , 0 1 5}$ \\
\hline Gruppe x Zeit & 0,20504 & 4,00 & 19,00 & 1,22512 & 0,333 \\
\hline
\end{tabular}

Tab. 3.17: Multivariate Varianzanalyse über tatsächliche Schlafzeit TST und Einschlaflatenz EL der VIS$M$ in den Messzeiträumen BL, S2, S9

Es gibt keine signifikanten Unterschiede zwischen den Gruppen vor Behandlungsbeginn, im zeitlichen Verlauf zeigen sich jedoch für beide Gruppen signifikante Unterschiede (Tab. 3.17).

In beiden Gruppen steigt die subjektive TST von der Baseline bis zum Therapiebeginn (S2) in etwa gleichem Maße an, in der IPT-I-Gruppe von 345,18 auf 366,39 min, in der PMR-Gruppe von „334,54 Minuten“ (zit. nach Müller-Popkes 1997, S. 136) auf 356,75 min. Während sich die subjektive TST der IPT-I-Gruppe vom Behandlungsbeginn bis zur 9. Sitzung nicht mehr verändert (365,15 min), kann die PMR-Gruppe sie um weitere 20 min auf 376,63 min steigern (s. Messzeitraum 9, Abb. 3.09, S. 81).

Die IPT-I-Patienten schlafen zum Therapiebeginn S2 (35,45 min) schneller ein als noch zur Baseline (47,2 min). Unter der Behandlung verkürzt sich die Einschlaflatenz weiter 
auf 24,85 min zur neunten Therapiesitzung. Auch die PMR-Patienten können von der Baseline zu Behandlungsbeginn ihre Einschlaflatenz senken: von „41,95 Minuten“ (zit. nach Müller-Popkes 1997, S. 136) auf 28,65 min. Nach der neunten Sitzung benötigen die PMR-Patienten mit 27,38 min noch praktisch die gleiche Zeit, um einzuschlafen (s. Messzeitraum 9, Abb.3 .10, S. 82).

Die subjektive Schlafquantität, die u.a. die Einschlaflatenz (EL) und die tatsächliche Schlafzeit (TST) beinhaltet, verändert sich somit in beiden Gruppen positiv: Bei Betrachtung der Zahlenwerte wird erkennbar, dass der signifikante Zeiteffekt zum einen auf einer Verbesserung von beiden Gruppen in beiden untersuchten Parametern von der Baseline zum Zeitraum vor der zweiten Therapiesitzung beruht. Zum anderen verbessert sich unter der Therapie bis zur neunten Sitzung in der IPT-I-Gruppe die Einschlaflatenz, in der PMR-Gruppe die TST.

Aufgrund der in der Multivariaten Varianzanalyse (Tab. 3.17) dargestellten Signifikanz im zeitlichen Verlauf wurde die Signifikanz der Veränderungen von der Baseline bis zur neunten Therapiesitzung in einem zweiten Auswertungsschritt überprüft. Hierzu wurden für jede Behandlungsgruppe und jede Variable getrennt zweiseitige verbundene t-Tests durchgeführt: (1. TST: IPT-I: p=0,20872; PMR: p=0,01892; 2. EL: IPT-I: p=0,01157; PMR: 0,234409).

Zur Beurteilung der Veränderung der subjektiven Schlafquantität der vergangenen Nacht von der Baseline bis zur neunten Therapiesitzung werden die in Tabelle 3.17 dargestellten Variablen TST und EL Bonferoni-adjustiert (Signifikanzniveau p=0,025, s. Kapitel 2.5).

Die Signifikanz der zunächst durchgeführten Multivariaten Varianzanalyse und die Ergebnisse der anschließend durchgeführten t-Tests sind der folgenden Übersichtstabelle zu entnehmen. 


\section{Übersicht Schlafquantität nach acht Wochen ( VIS-M )}

\begin{tabular}{|l|l|l|l|l|l|}
\hline \multirow{2}{*}{$\begin{array}{l}\text { Variable } \\
\text { im VIS-M }\end{array}$} & \multirow{2}{*}{ 1.Test } & Signifikanzen & 2.Test & \multicolumn{2}{|c|}{ Einzelergebnisse der Gruppen } \\
\cline { 5 - 6 } & & & & IPT-I & \multicolumn{2}{|c|}{ PMR } \\
\hline TST & MvVa & ZE & t-Test(gepaart) & $p=0,20872$ & $\mathbf{p}=\mathbf{0 , 0 1 8 9 2}$ \\
EL & & & t-Test(gepaart) & $\mathbf{p = 0 , 0 1 1 5 7}$ & $\mathrm{p}=0,234409$ \\
\hline
\end{tabular}

Tab. 3.18: VIS-M - subjektive Schlafquantität in der täglichen Beurteilung zwischen den Messzeiträumen Baseline und S9, quantitative Parameter: TST = tatsächliche Schlafzeit, EL

= Einschlaflatenz. $M v V a=$ Multivariate Varianzanalyse. $\mathrm{ZE}=$ Zeiteffekt.

Die Veränderung der Einschätzung der vergangenen Nacht schon nach acht Wochen zeigt sich hinsichtlich der Schlafquantität für beide Behandlungsgruppen,

da die IPT-I-Gruppe ihre Einschlaflatenz, die PMR-Gruppe ihre TST signifikant verbessern kann.

Die Veränderung der qualitativen Parameter Schlafqualität (SQ) und morgendliches Erholungsgefühl (mE) wird analog zu den quantitativen Parametern geprüft:

Univariate Varianzanalyse SQ (VIS-M, 3 Messzeitpunkte)

\begin{tabular}{|c|c|c|c|c|c|}
\hline Varianzquelle & SS & df & MS & $\mathbf{F}$ & Sign. \\
\hline Zwischen den Vpn & 9437,90 & 22 & 429,00 & & \\
\hline Gruppe & 434,24 & 1 & 434,24 & 1,01 & 0,325 \\
\hline Varianzquelle & Wert & Hyp. df & Fehl. df & $\mathbf{F}$ & Sign. \\
\hline Zeit & 0,33715 & 2,00 & 21,00 & 5,34067 & 0,013 \\
\hline Gruppe x Zeit & 0,04991 & 2,00 & 21,00 & 0,55161 & 0,584 \\
\hline
\end{tabular}

Tab. 3.19: Univariate Varianzanalyse über Schlafqualität SQ der VIS-M in den Messzeiträumen BL, S2, S9. Vpn = Versuchspersonen

Betrachtet man die Schlafqualität über die drei entsprechenden Messzeitpunkte, so zeigt die Baseline beider Gruppen keinen signifikanten Unterschied, es ist jedoch ein Zeiteffekt erkennbar. 
Bei der Betrachtung der Mittelwerte (s. Abb. 3.15, S.93) wird deutlich, dass beide Gruppen ihre im mittleren Bereich befindliche subjektive Schlafqualität etwas verbessern können. Auf der visuellen Analogskala, der in der Auswertung nachträglich eine Einteilung von eins bis 100 zugewiesen wurde, verbessert sich die subjektive Schlafqualität von der Baseline bis zur zweiten Sitzung in der IPT-I-Gruppe von 43,96 auf 50,00, in der PMR-Gruppe von „46,65“ (zit. nach Müller-Popkes 1997, S. 137) auf 53,13. Im Verlauf der Behandlung können die IPT-I-Patienten bis zur neunten Sitzung mit 50,27 keine weitere Steigerung erreichen, dagegen schätzen die PMRPatienten zum neunten Termin mit 59,18 die Qualität ihrer Nächte noch höher ein als zu Therapiebeginn.

Auch für die Schlafqualität wurde die Signifikanz der Veränderungen von der Baseline bis zur neunten Therapiesitzung in einem zweiten Auswertungsschritt überprüft. Hierzu wurden für jede Behandlungsgruppe getrennt zweiseitige verbundene $t$-Tests durchgeführt: (SQ: IPT-I: p=0,18788; PMR: p=0,02575).

Der zweite qualitative Schlafparameter, das morgendliche Erholungsgefühl (mE) wurde ebenfalls für einen verkürzten Therapiezeitraum berechnet:

Univariate Varianzanalyse mE (VIS-M, 3 Messzeitpunkte)

\begin{tabular}{|c|c|c|c|c|c|}
\hline Varianzquelle & SS & df & MS & F & Sign. \\
\hline Zwischen den Vpn & 11822,03 & 22 & 537,37 & & \\
\hline Gruppe & 903,27 & 1 & 903,27 & 1,68 & 0,208 \\
\hline \multicolumn{7}{|l|}{} \\
\hline Varianzquelle & Wert & Hyp. df & Fehl. df & F & Sign. \\
\hline Zeit & 0,11287 & 2,00 & 21,00 & 1,33596 & 0,284 \\
\hline Gruppe x Zeit & 0,10996 & 2,00 & 21,00 & 1,29716 & 0,294 \\
\hline
\end{tabular}

Tab. 3.20: Univariate Varianzanalyse über morgendliches Erholungsgefühl mE der VIS-M in den Messzeiträumen BL, S2, S9. Vpn = Versuchspersonen.

In der Beurteilung des morgendlichen Erholungsgefühls unterscheiden sich die Gruppen in der Baseline nicht.

Bei Betrachtung der Mittelwerte der drei Messzeiträume der einzelnen Gruppen ist zu beachten, dass eine Abnahme der Werte eine Besserung des mE bedeutet (s. Abb. 3.16, 
S 94). Das morgendliche Erholungsgefühl der IPT-I-Gruppe verändert sich praktisch nicht (BL: 56,04; S2: 54,51; S9: 57,13), Das morgendliche Erholungsgefühl der PMR-Gruppe bessert sich hingegen tendenziell von der Baseline zur zweiten Sitzung von „54,16“ (zit. nach Müller-Popkes 1997, S. 137) auf 46,6, dieser Effekt hält sich mit 45,66 zur neunten Sitzung, diese Veränderungen über die Zeit sind jedoch nicht signifikant.

Für das morgendliche Erholungsgefühl wurde wegen der fehlenden Signifikanz ein zweiter Auswertungsschritt über Veränderungen von der Baseline bis zur neunten Therapiesitzung mittels t-Tests nicht durchgeführt.

Zur Beurteilung der Veränderung der qualitativen Parameter der vergangenen Nacht von der Baseline bis zur neunten Therapiesitzung werden die in Tabelle 3.21 dargestellten Variablen SQ und mE Bonferoni-adjustiert (Signifikanzniveau p=0,025, s. Kapitel 2.5).

Die Signifikanzen der zunächst durchgeführten Univariaten Varianzanalysen und die Ergebnisse der anschließend durchgeführten zweiseitigen t-Tests für abhängige Stichproben sind der folgenden Tabelle zu entnehmen.

\section{Übersicht qualitative Parameter nach acht Wochen (VIS-M)}

\begin{tabular}{|c|c|c|c|c|c|}
\hline \multirow{2}{*}{$\begin{array}{l}\text { Variable } \\
\text { im VIS-M }\end{array}$} & \multirow{2}{*}{ 1.Test } & \multirow{2}{*}{ Signifikanzen } & \multirow{2}{*}{ 2.Test } & \multicolumn{2}{|c|}{ Einzelergebnisse der Gruppen } \\
\hline & & & & IPT-I & PMR \\
\hline $\begin{array}{l}\mathrm{SQ} \\
\mathrm{mE}\end{array}$ & $\begin{array}{l}\text { UvVa } \\
\text { UvVa }\end{array}$ & ZE & $\begin{array}{l}\text { t-Test(gepaart) } \\
\text { n.d. }\end{array}$ & $p=0,18788$ & $p=0,02575$ \\
\hline
\end{tabular}

Tab. 3.21: qualitative Parameter in der Beurteilung der vergangenen Nacht zwischen den Messzeiträumen Baseline und S9. SQ = Schlafqualität, $m E=$ morgendliches Erholungsgefühl. $U v V a=$ Univariate Varianzanalyse, $Z E=Z e i t e f f e k t, n . d .=2$. Test wurde mangels Signifikanz im 1. Test nicht durchgeführt.

Die Veränderung der Einschätzung der vergangenen Nacht schon nach acht Wochen zeigt sich hinsichtlich der qualitativen Parameter für keine der beiden Behandlungsgruppen, die PMR-Gruppe liefert jedoch ein grenzwertiges Ergebnis, das auf der Verbesserung der Schlafqualität beruht.

Unter der jeweiligen Therapie kommt es von der zweiten bis zur neunten Sitzung bei der IPT-I-Gruppe zu einer Abnahme der Einschlaflatenz, bei der PMRGruppe zu einer Zunahme der TST und grenzwertig signifikant der Schlafqualität. 


\subsection{Anhang zur Verlaufsbetrachtung: Darstellung eingebetteter Daten}

Der Gesamtverlauf des Schlafes während der Behandlungsdauer von 12 Wochen an Hand der täglich morgens ausgefüllten VIS-M-Fragebögen wurde bereits dargestellt (s. Kapitel 3.4). In den Fragebögen bewerteten die Patienten den Schlaf der vergangenen Nacht zu Hause. Wie bereits in Kapitel 3.4 dargestellt, wurden für jede der den Behandlungsterminen 2 bis 12 vorhergehenden Wochen fünf Nachtprotokolle der einzelnen Patienten zu einem Mittelwert zusammengefasst. Anschließend wurde aus den Mittelwerten der Patienten ein Mittelwert für die jeweilige Behandlungsgruppe gebildet.

Um von einem sowohl für diese vorliegende Untersuchung als auch für die gesamte Studie verbindlichen Ausgangswert vor der Behandlung ausgehen zu können, wurde in den folgenden grafischen Darstellungen (Abb. 3.13 - 3.16) der Reihe der von der Verfasserin der vorliegenden Untersuchung ermittelten Messwerte 2 bis 12 der Wert Baseline (BL) vorangestellt. Dieser Baseline-Wert (BL) wurde bereits von MüllerPopkes 1997 veröffentlicht. Er ist ein Mittelwert aus 14 VIS-M-Fragebögen für den zwischen dem Erstgespräch und der Eingangspolysomnografie liegenden Zeitraum von zwei Wochen häuslichen Schlafes. Um zu demonstrieren, wie die Nächte im Schlaflabor im Verhältnis zu den Nächten in häuslicher Umgebung bewertet wurden, sind die Messzeitpunkte der Eingangs- und Kontrollpolysomnografien mit abgebildet. Die diesen Messzeitpunkten zugrunde liegenden Daten für die PMR-Gruppe wurden bereits veröffentlicht (Müller-Popkes 1997), die Daten der IPT-I-Gruppe sind jedoch nicht mit denen von Müller-Popkes 1997 identisch, da aus der IPT-I-Gruppe für die Verlaufsbetrachtung die Daten eines Patienten eliminiert, wurden, der Extremwerte lieferte. Dieser Patient war zugleich der älteste Patient der gesamten Stichprobe, der aus dem zunächst vorgesehenen Altersbereich herausfiel. Die IPT-I-Werte wurden nach eigenen Rohdaten von der Verfasserin der vorliegenden Untersuchung neu berechnet. Die dahingehend bereinigten Mittelwerte der 14 Messzeiträume sind in den folgenden Abbildungen für die subjektiven Parameter tatsächliche Schlafzeit (TST), Einschlaflatenz (EL), Schlafqualität (SQ) und morgendliches Erholungsgefühl (mE) dargestellt. 


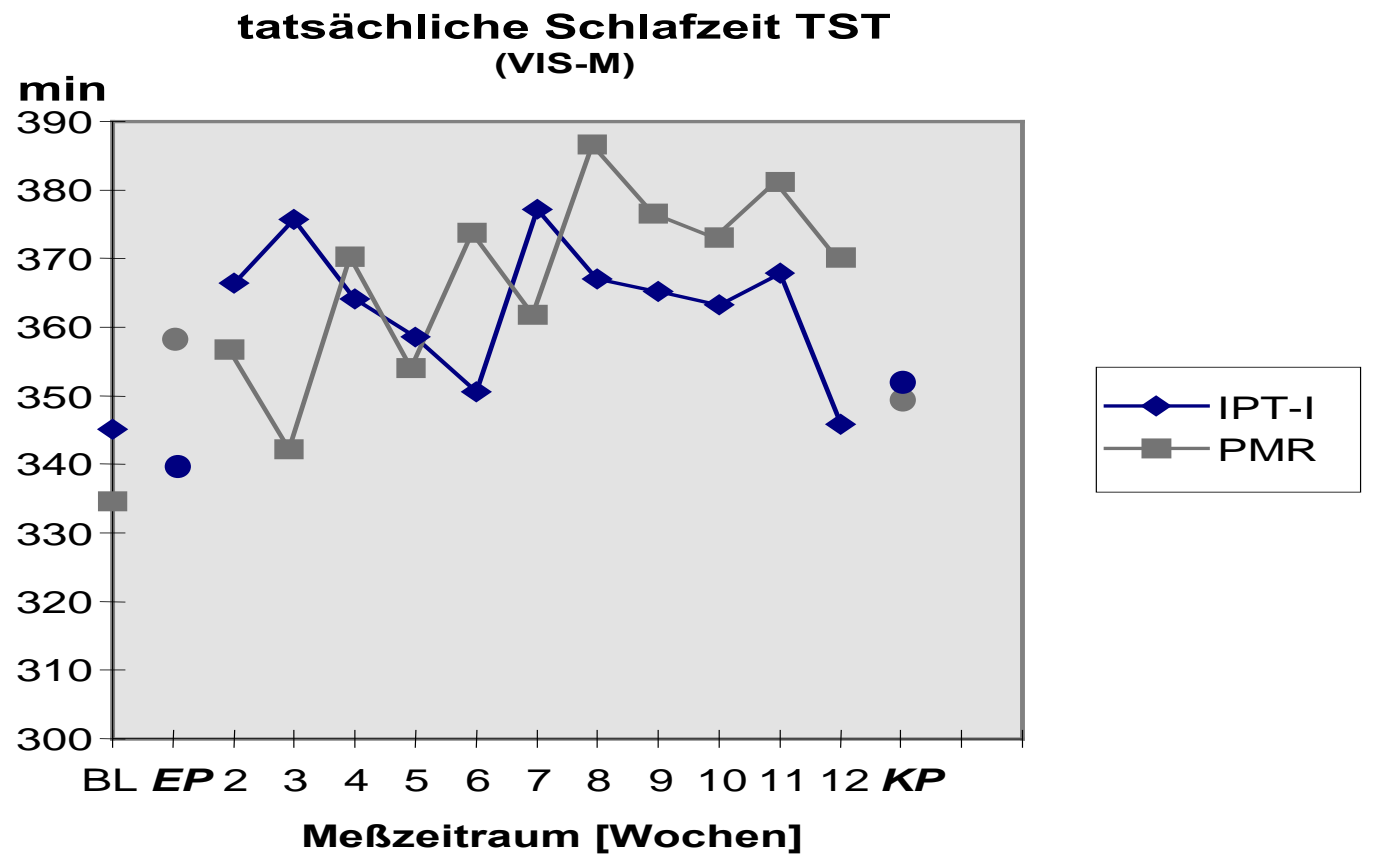

Abb. 3.13: 1. eingebettete Verlaufsbetrachtung VIS-M: tatsächliche Schlafzeit (TST) in Minuten. 2-12 = wöchentliche Therapiesitzungen. Die Abszissenachse ist nicht proportional. (Rahmendaten BL, EP, KP der PMR: bereits veröffentlicht Müller-Popkes 1997. Die Mittelwerte der jeweils zwei Nächte vor (EP) und nach (KP) der Behandlung im Schlaflabor sind hier durch kreisförmige Messpunkte dargestellt).

1. tatsächliche Schlafzeit (TST): Im Schlaflabor vor Therapiebeginn (EP) schläft die PMR-Gruppe bereits deutlich länger, die IPT-I-Gruppe dagegen etwas kürzer als zur Baseline. Zu Behandlungsbeginn (2) schlafen beide Gruppen gegenüber der Baseline mehr als 20 Minuten länger. Die PMR-Gruppe kann während der Behandlung unter Schwankungen insgesamt einen leichten kontinuierlichen Anstieg verzeichnen und erzielt überwiegend längere subjektive tatsächliche Schlafzeiten als die IPT-I-Gruppe, die im mittleren Teil der Therapie (6) etwas einbricht. Nach der elften Sitzung ist bei beiden Gruppen ein in der IPT-I-Gruppe sehr deutlicher Rückgang zu verzeichnen. Zum letzten Behandlungstermin liegt in der PMR-Gruppe eine deutliche Verbesserung um mehr als eine halbe Stunde im Vergleich zur Baseline vor, in der abschließenden Kontrollpolysomnografie ist diese jedoch wieder auf die Hälfte zusammengeschmolzen. In der IPT-I-Gruppe kommt es durch den Einbruch zu keiner Verbesserung gegenüber der Baseline, in der abschließenden Kontrolle im Schlaflabor (KP) wieder zu einer leichten Verbesserung. 


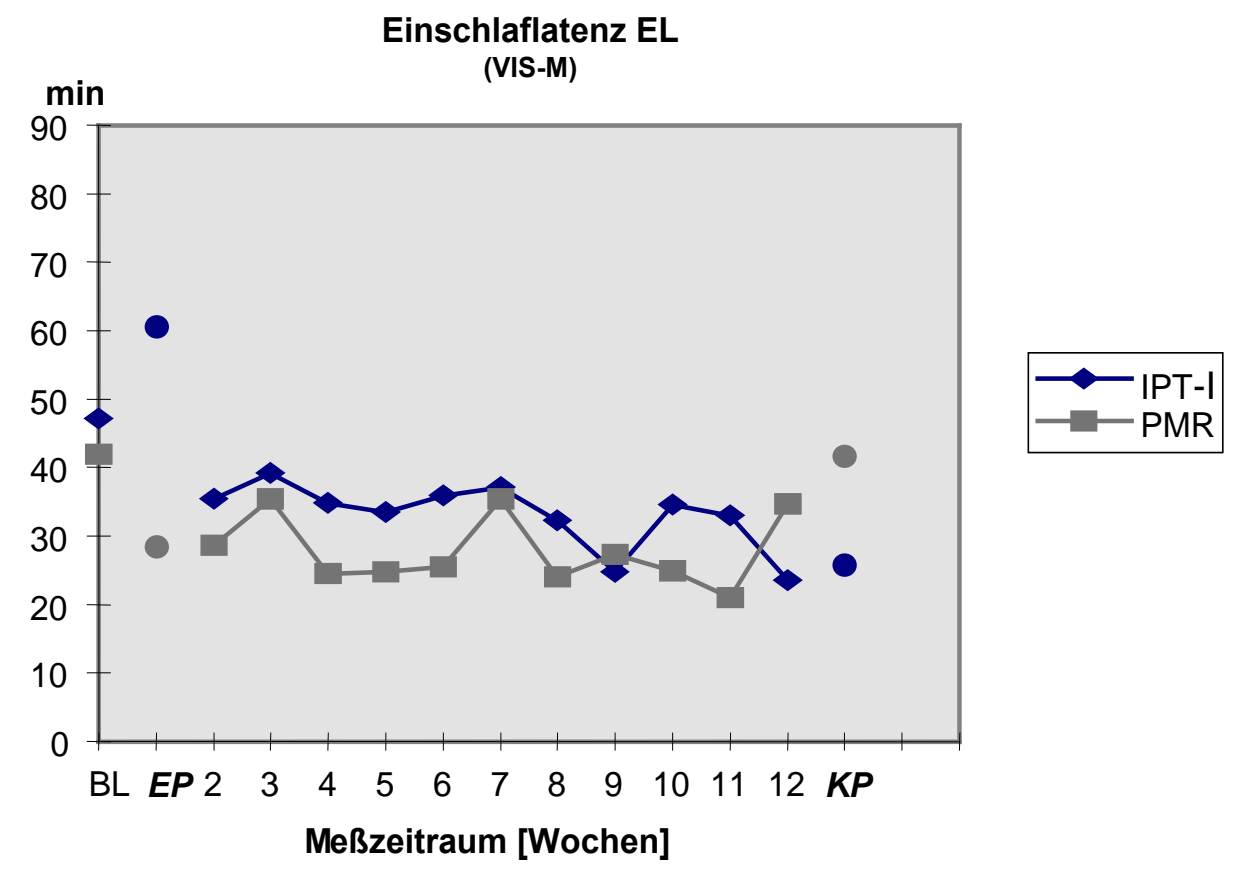

Abb. 3.14: 2. eingebettete Verlaufsbetrachtung VIS-M: Einschlaflatenz (EL) in Minuten. Kreis = EL von IPT-I bzw. PMR im Schlaflabor. 2-12 = wöchentliche Therapiesitzungen. Die Abszissenachse ist nicht proportional. (Rahmendaten BL, EP, KP der PMR: bereits veröffentlicht Müller-Popkes 1997. Die Mittelwerte der jeweils zwei Nächte vor (EP) und nach (KP) der Behandlung im Schlaflabor sind hier durch kreisförmige Messpunkte dargestellt.)

2. Einschlaflatenz (EL): Die Einschlaflatenzen der IPT-I- und der PMR-Gruppe nehmen von der Baseline zum eigentlichen Beginn der jeweiligen Therapiesitzungen (2) jeweils um mehr als 10 Minuten ab. Die IPT-I-Gruppe hat dazwischen in der Eingangspolysomnografie (EP) eine mit über 60 Minuten sehr hohe Einschlaflatenz, die PMR-Gruppe kann diese dagegen bereits vor Behandlungsbeginn bis zum Zeitpunkt der Eingangspolysomnografie deutlich reduzieren. In der IPT-I-Gruppe liegen die Einschlaflatenzen während der ersten Sitzungen mit ihren Schwankungen etwas über denen der PMR-Gruppe, ab der achten Sitzung gleichen sie sich denen an und erreichen zum letzten Sitzungstermin im Vergleich zur PMR-Gruppe, die etwas ansteigt, einen besseren Wert. Die PMR-Gruppe verbessert sich dagegen im Verleich zur Baseline nur gering und nach dem Wert der abschließenden Untersuchung im Schlaflabor (KP) kaum. 


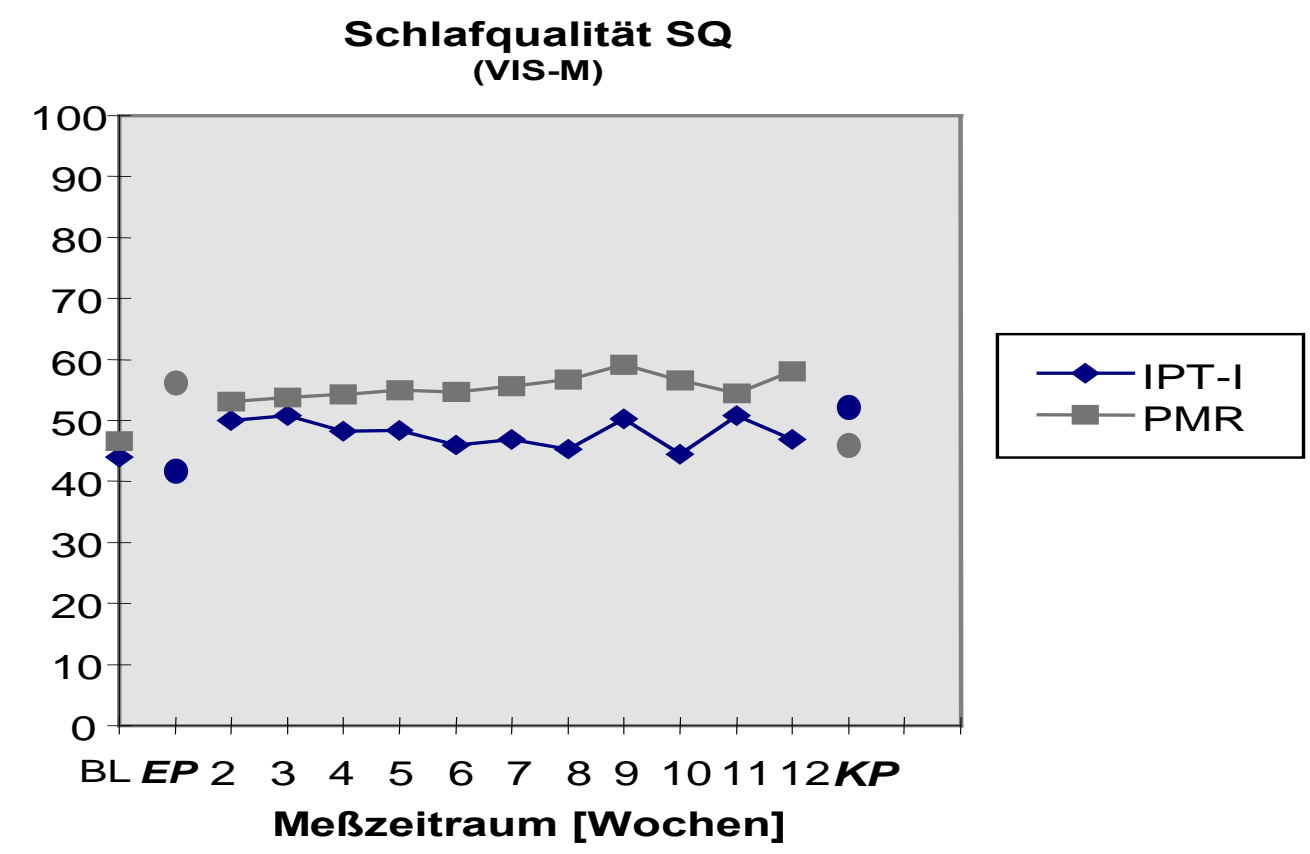

Abb. 3.15: 3. eingebettete Verlaufsbetrachtung VIS-M: Schlafqualität (SQ); $0=$ sehr schlechte Nacht, $100=$ sehr gute Nacht, Kreis = SQ von IPT-I bzw. PMR im Schlaflabor. 2-12 = wöchentliche Therapiesitzungen. Die Abszissenachse ist nicht proportional. (Rahmendaten BL, EP, KP der PMR: bereits veröffentlicht Müller-Popkes 1997. Die Mittelwerte der jeweils zwei Nächte vor (EP) und nach (KP) der Behandlung im Schlaflabor sind hier durch kreisförmige Messpunkte dargestellt.)

3. Schlafqualität (SQ): Die subjektive Schlafqualität liegt bereits zur Baseline bei beiden Gruppen noch im mittleren Bereich. Zunächst bessert sich die subjektive Schlafqualität von der Baseline bis zum Therapiebeginn gleichermaßen leicht, ist jedoch in der PMR-Gruppe zum Zeitpunkt der Eingangspolysomnografie (EP) noch besser als vor dem eigentlichen Behandlungsbeginn (2). Ab der dritten Sitzung verhalten sich die Gruppen gegensätzlich: Die IPT-I-Gruppe fällt unter leichten Schwankungen fast auf ihren Baselinewert zurück. Die PMR-Gruppe weist in ihrer zunächst kontinuierlichen Verbesserung ab der neunten Sitzung sehr leichte Schwankungen auf. Insgesamt nehmen die Schwankungen in beiden Gruppen im Verlauf etwas zu. Gegen Behandlungsende weichen beide Gruppen etwas auseinander, wobei sich nur die PMRGruppe im Vergleich zur Baseline deutlich verbessern kann. Zum Zeitpunkt der Kontrollpolysomnografie fällt die Schlafqualität in der PMR-Gruppe wieder deutlich ab und steigt dagegen in der IPT-I-Gruppe. 


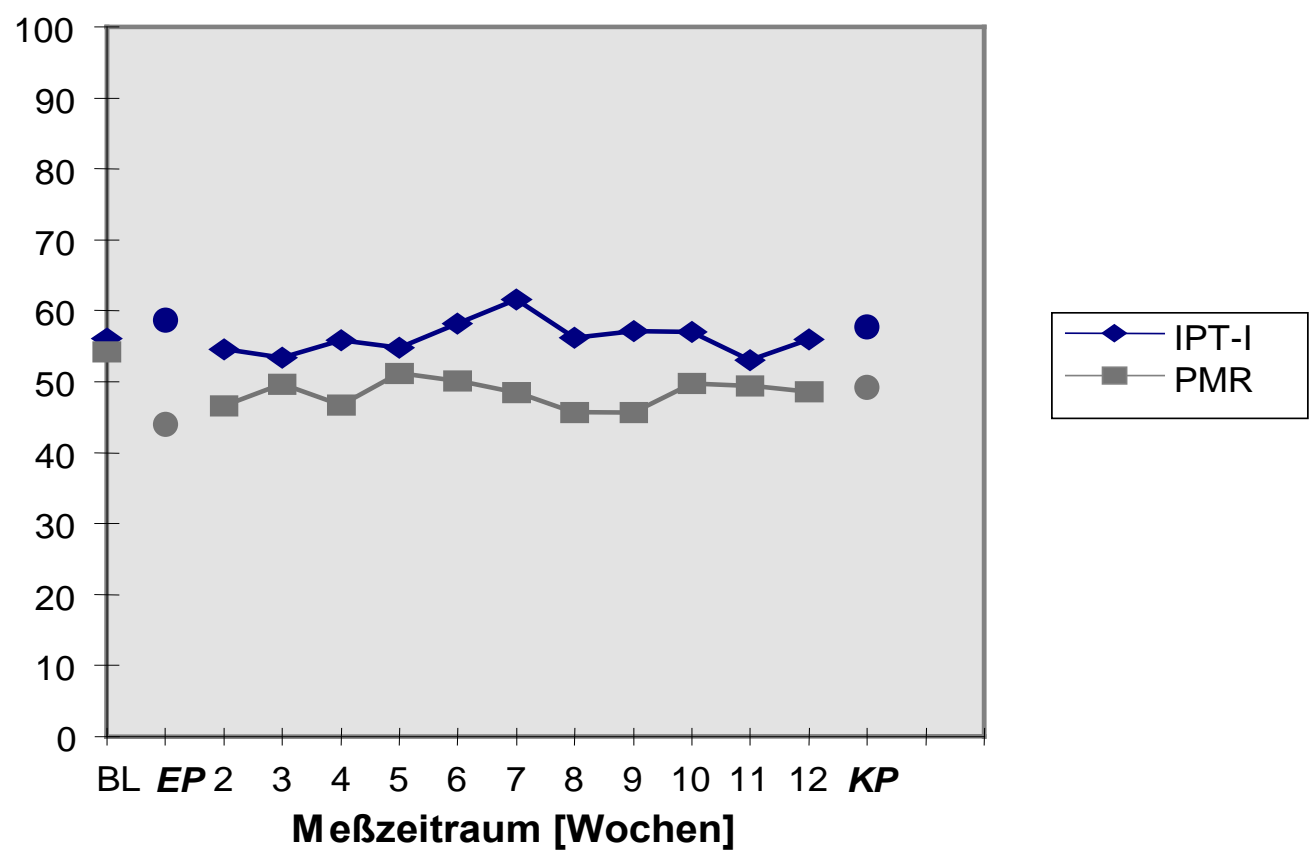

Abb. 3.16: 4. eingebettete Verlaufsbetrachtung VIS-M: morgendliches Erholungsgefühl ( $\mathrm{mE}) ; 0=$ wundervoll frisch und energiegeladen, 100= schrecklich müde und lustlos. Kreis = mE von IPT-I bzw. PMR im Schlaflabor. 2-12 = wöchentliche Therapiesitzungen. Die Abszissenachse ist nicht proportional. (Rahmendaten BL, EP, KP der PMR: bereits veröffentlicht MüllerPopkes 1997. Die Mittelwerte der jeweils zwei Nächte vor (EP) und nach (KP) der Behandlung im Schlaflabor sind hier durch kreisförmige Messpunkte dargestellt.)

4. morgendliches Erholungsgefühl (mE): Die zur Baseline dicht beieinanderliegenden Werte verschlechtern sich zur Eingangspolysomnografie leicht in der IPT-I-Gruppe und verbessern sich deutlich in der PMR-Gruppe, diese gegensätzlichen Veränderungen liegen zu Therapiebeginn in der PMR-Gruppe etwas abgeschwächt, in der IPT-I-Gruppe nicht mehr vor. Im mittleren Bereich der Behandlung schwanken beide Gruppen, die IPT-I-Gruppe stärker, sie erreicht hier einen Tiefpunkt ihres morgendlichen Erholungsgefühls. In der PMR-Gruppe kommt es von der ersten eigentlichen Therapiesitzung bis zur letzten insgesamt zu keinen weiteren Verbesserungen des durchweg im mittleren Bereich bzw. leicht darüber liegenden und damit im Vergleich zur IPT-I-Gruppe durchweg besseren morgendlichen Erholungsgefühls. Im Vergleich zur Baseline fühlt sich die PMR-Gruppe am Behandlungsende jedoch insgesamt etwas besser erholt, während die IPT-I-Gruppe keine wesentliche Verbesserung erreichen kann. 


\section{Diskussion}

Ist Interpersonelle Psychotherapie, hier im Vergleich mit der bereits vielfach bewährten Progressiven Muskelrelaxation nach Jacobson, eine wirksame Behandlungsmethode bei Patienten mit primärer Insomnie? Nach den bislang vorliegenden Ergebnissen (MüllerPopkes 1997) muss diese Frage verneint werden. Es sollte daher zunächst eine umfassendere Auswertung unter Berücksichtigung zusätzlicher Fragebögen erfolgen, die während der Aufenthalten im Schlaflabor vor und nach der Behandlung ausgefüllt worden waren und Auskunft gaben über den subjektiven Schlaf der vergangenen zwei Wochen sowie der zurückliegenden Nacht.

Bereits in der bisherigen Auswertung (Müller-Popkes 1997) wurde die Notwendigkeit einer speziellen Verlaufsbetrachtung gesehen, um „spezifischere Aussagen über die Wirkkomponenten der beiden Behandlungsformen“ (Müller-Popkes 1997, S. 165) treffen zu können. Bei der in der vorliegenden Untersuchung vorgenommenen Verlaufsbetrachtung zeigte sich jedoch die deutliche Abweichung der subjektiven Beurteilung des Schlafes im Schlaflabor von den Beurteilungen des Schlafes in gewohnter Umgebung. Das bedeutet, dass die Patienten zu Hause entweder anders schliefen als im Schlaflabor oder den Schlaf im Schlaflabor anders beurteilten als den Schlaf in der gewohnten häuslichen Umgebung. Die Messzeitpunkte der Ein- und Kontrollpolysomnografienächte stellten sich als für die Beurteilung des Therapieeffektes wenig geeignet heraus, da sie nicht repräsentativ waren.

Der aufgrund des hohen Zeitaufwandes vor allem der IPT-I interessanten Fragestellung, ob sich die Behandlung ohne Wirkungsverlust um ein Drittel auf acht Sitzungen verkürzen ließe, wurde in der vorliegenden Untersuchung nachgegangen. Da sich, wie bereits im vorangegangenen Absatz dargestellt, der subjektive Schlaf im Schlaflabor von dem der häuslichen Nächte unterschied, fand er hierbei keinen Eingang in die Berechnung. Die Vorgehensweise und die Ergebnisse der vorliegenden Untersuchung, auch im Bezug auf die bereits gewonnenen Erkenntnisse (Müller-Popkes 1997), sind Gegenstand der folgenden Diskussion.

\subsection{Patientenrekrutierung und Zusammensetzung der Stichprobe}

Die Patienten wurden überwiegend durch Rundfunk und Zeitung rekrutiert. Diese Vorgehensweise birgt das Risiko, vorwiegend Patienten für die Studie zu gewinnen, die 
nur leicht schlafgestört sind (Paterok 1993). Diese Patienten hätten sich vermutlich ohne den äußeren Anstoß durch die Medien nicht in schlaftherapeutische Behandlung begeben. Es waren jedoch ausdrücklich chronisch Schlafgestörte über die Medien angesprochen und über eine nichtmedikamentöse Therapie informiert worden. Der Bekanntheitsgrad nichtmedikamentöser Therapieformen zur Behandlung von Schlafstörungen ist sowohl bei Patienten als auch bei Hausärzten gering (Müller-Popkes 1997). Die durchschnittliche Krankheitsdauer der vorliegenden Stichprobe entsprach den Patienten, die sich von sich aus in Behandlung begeben (Jacobs et al. 1996). Für viele schwerst Schlafgestörte wäre der für die Aufnahme in diese Studie notwendige Verzicht auf Schlafmedikation ein unüberwindbares Problem gewesen. Erst nach einem erfolgreichen Medikamentenentzugsprogramm könnten solche Patienten in die Studie aufgenommen werden (Espie 1991). Die Voraussetzung, den Verzicht auf Medikamente einzuhalten, bedingt eine Selektion auf Patienten, die sich bewusst für eine aktive Behandlungsform ihres Schlafproblems entscheiden (Müller-Popkes 1997). Der Altersdurchschnitt der Stichprobe ist mit 47 Jahren repräsentativ für chronisch Schlafgestörte, obwohl mit höherem Alter die Prävalenz noch weiter ansteigt (Simen et al. 1996). Frauen sind von Insomnie häufiger betroffen (Mellinger et al. 1985), der Frauenanteil war daher in der Stichprobe zu gering. Das Ziel der Untersuchung war jedoch nicht, den bzw. die typische Insomniepatientin zu behandeln, sondern mit einer Stichprobe zu arbeiten, die dem Bevölkerungsdurchschnitt entspricht. Daher ist sie sehr heterogen sowohl bezüglich demographischer Variablen als auch in Art und Dauer der Schlafstörung.

\subsection{Studiendesign}

Es gab keine Kontrollgruppe, da es zum einen ethisch fragwürdig erschien, eine Patientengruppe über mehr als drei Monate unbehandelt zu lassen, zum anderen hätten sich die Patienten während dieser Zeit wahrscheinlich anderweitig medikamentöse oder andere therapeutische Hilfe gesucht (Müller-Popkes 1997). In vorliegenden Studien hat sich gezeigt, dass sich unbehandelte Kontrollgruppen nicht spontan verbessern (Murtagh und Greenwood 1995, Borkovec und Hennings 1978, Bootzin und Nicassio 1978, Turner und Ascher 1979, Nicassio et al. 1982, Woolfolk und McNulty 1983). Aus diesen Gründen schien es gerechtfertigt, auf eine unbehandelte Kontrollgruppe zu verzichten.

Die Zuteilung der Patienten zu den Behandlungsgruppen erfolgte randomisiert. Dies könnte verhindert haben, dass diejenigen Patienten, die sich für eine der beiden 
Behandlungsgruppen besonders eignen, auch den entsprechenden Gruppen zugewiesen wurden (Turner und Ascher 1979). Für die Vergleichbarkeit der Gruppen bei dieser geringen Größe wäre es möglicherweise sinnvoll gewesen, statt einer Zufallsverteilung die Patienten nach soziodemographischen Parallelitätskriterien wie Alter, Geschlecht und sozioökonomischem Status (Paterok 1993) zuzuteilen.

In der vorliegenden Untersuchung fanden längere Nachbeobachtungszeiträume keine besondere Beachtung, da sie sich insbesondere der Verlaufsbetrachtung während der Therapie widmet.

\subsection{Auswertung}

Die vorgenommene Auswertung lässt unberücksichtigt, ob die für die einzelnen Gruppen festgestellten Änderungen auf geringfügigen Veränderungen aller Patienten der entsprechenden Gruppe beruhen, oder ob einige Patienten sich deutlich verbessern, während andere sich therapierefraktär zeigen (Garfield 1981). Bei dem vorliegenden, sehr heterogenen Patientengut könnte eine Individualauswertung interessante Ergebnisse liefern. Da Ein- und Durchschlafstörungen bei den gleichen Patienten wechseln können (Hohagen et al. 1994), kann auf eine Aufteilung in Ein- und Durchschlafstörung verzichtet werden. Die statistische Auswertung würde durch eine weitere Unterteilung in Subgruppen bei einer ohnehin kleinen Stichprobengrößen sehr erschwert sein. Größere Stichproben wiederum standen aus personellen und Kapazitätsgründen nicht zur Verfügung. Bei der vorliegenden Patientenzahl konnten beide zeitlich aufwendige Behandlungen von derselben Therapeutin durchgeführt werden und sind daher besser vergleichbar.

Die Entscheidung, für die Verlaufsbetrachtung und für die Untersuchung einer möglichen Kürzung der Behandlungsdauer einen Ausreißer aus der Auswertung zu nehmen, der der älteste Patient war, ist vertretbar, es wäre jedoch besser gewesen, initial die Altersgrenze der Stichprobe für den Einschluss in die Studie auf 65 Jahre zu senken, da sich der Schlaf im Alter deutlich verändert: Nach einer Meta-Analyse von Floyd et al. 2000 nimmt im Alter die Häufigkeit und die Dauer nächtlichen Wachseins zu, ebenso nehmen die nächtliche Schlafmenge und die Fähigkeit einzuschlafen ab; bei polysomnografischen Messungen wurden größere altersbedingte Änderungen gefunden als durch Selbstbeurteilungen. Eine altersbedingte Veränderung thalamocorticaler Regulationsmechanismen ist möglicherweise die Ursache für eine altersbedingte Abnahme der Dichte der für die Einstufung in das Schlafstadium 2 (Rechtschaffen und Kales 1968) bedeutsamen Schlafspindeln und K-Komplexe (Crowley et al. 2002). 


\subsection{Therapeutische Effekte}

\subsubsection{Subjektive Schlafparameter}

Die Veränderung der subjektiven Parameter wurde für unterschiedliche Bewertungszeiträume untersucht. Die Aussagekraft von globalen Bewertungen eines längeren Zeitraumes im Vergleich zu allmorgendlich ausgefüllten Protokollen einer vorangegangenen Nacht wird in der Literatur widersprüchlich beurteilt. Danach neigen die Patienten einerseits dazu, auf Behandlungseffekte weniger sensibel zu reagieren und unterliegen einer stärkeren Tendenz, zur Erfüllung der eigenen und der Erwartungshaltung des Therapeuten die Fragebögen beschönigend auszufüllen (Bootzin und Nicassio 1978). Es könnte andererseits jedoch eine schlechtere Bewertung vorliegen, da Patienten schlechtere Nächte besser im Gedächtnis behalten als gute (Mendelson 1987). Aufgrund dieser widersprüchlichen Einschätzung ist es ein Vorzug der vorliegenden Untersuchung, über Daten bezüglich unterschiedlicher Bewertungszeiträume zu verfügen, wobei neben der täglichen Einschätzung der längere Beurteilungszeitraum aufgrund der oben genannten Gründe auf zwei Wochen beschränkt wurde.

\subsubsection{Die Schlafbewertung eines zweiwöchigen Zeitraumes}

\subsubsection{1 quantitative Parameter}

Die Daten der quantitativen Parameter zur Beurteilung eines zweiwöchigen Zeitraumes wurden im SF-B vor und nach der Behandlung während der Aufenthalte im Schlaflabor erhoben. Die subjektive Einschlaflatenz verminderte sich nur in der PMR-Gruppe signifikant, die nächtliche Wachzeit reduzierte sich in beiden Gruppen signifikant. Da sich die Häufigkeit der Schlafunterbrechungen nicht signifikant änderte, ist davon auszugehen, dass die Patienten nach nächtlichem Erwachen schneller wieder einschliefen. In beiden Gruppen blieb eine signifikante Veränderung der tatsächlichen Schlafzeit aus. Wenn auch der Erfolg Haynes' et al. (1974) einer Reduktion der Aufwachfrequenz nicht wiederholt werden konnte, so gleicht die PMR dies mit der Verringerung der bisher kaum untersuchten nächtlichen Wachzeit wieder aus. Die Verringerung der nächtlichen Wachzeit für beide Gruppen ist ein Ergebnis, das aus Müller-Popkes 1997 nicht hervorging, denn über die Schlafquantität eines zweiwöchigen Zeitraumes gab es bislang keine Daten. Die Schlafeffizienz, die die nächtliche Wachzeit beinhaltet, konnte dort nur in der PMR-Gruppe verbessert werden (Müller-Popkes 1997). Die dort gezeigte Senkung der Einschlaflatenz in der IPT-IGruppe konnte widerum hier für einen zweiwöchigen Zeitraum nicht nachgewiesen 
werden. Die fehlende Auswirkung der IPT-I auf die Einschlaflatenz könnte möglicherweise dadurch erklärt werden, dass diese Patientengruppe vor dem Einschlafen über die interpersonellen Probleme grübelt, die sie auch zu Behandlungsende noch beschäftigen (Paterok 1993). Die nach bisherigen Untersuchungen (Steinmark und Borkovec 1974, Nicassio et al. 1982, s. Kapitel 1.3.2) erwartete Senkung der Einschlaflatenz wurde in der PMR-Gruppe bestätigt. Inwieweit die tendenziellen Verbesserungen durch die im Rahmen der IPT-I vermittelten schlafhygienischen Verhaltensweisen bedingt und damit unspezifische Effekte sind, kann mangels einer ausschließlich schlafhygienisch behandelten Kontrollgruppe nicht beurteilt werden. Bezüglich der quantitativen Schlafparameter in der Beurteilung eines Zweiwochenzeitraumes kann nach Auswertung mit erweitertem Datenmaterial die PMR in ihrer Wirksamkeit als gestärkt beurteilt werden durch die im Gegensatz zur IPT-I signifikante Senkung der Einschlaflatenz. Interessant ist weiterhin, dass beide Behandlungsgruppen eine signifikante Minderung ihrer nächtlichen Wachzeit erreichen konnten.

\subsubsection{2 qualitative Parameter}

auch die Daten der qualitativen Parameter zur Beurteilung eines zweiwöchigen Zeitraumes wurden im SF-B vor und nach der Behandlung während der Aufenthalte im Schlaflabor erhoben. Die vor der Behandlung schlechtere Schlafqualität der IPT-I- im Vergleich zur PMR-Gruppe ist bereits bei Müller-Popkes 1997 diskutiert worden, die einen angstauslösenden Effekt der bevorstehenden Therapie als Ursache annahm. Die Patienten der IPT-I-Gruppe sind möglicherweise über die reine Vermittlung schlafhygienischer Verhaltensweisen hinaus bereits in der ersten Sitzung, das heißt vor der Bearbeitung des Ausgangsfragebogens, durch die ausführliche Anamneseerhebung für einen möglichen Zusammenhang zwischen ihrer Schlafstörung und interpersonellen Problemen sensibilisiert worden. Die PMR ist in der mittelfristigen Beurteilung der qualitativen Parameter der IPT-I überlegen, um so mehr, als die IPT-I-Gruppe in der Ausgangsmessung schlechtere Werte in der Schlafqualität aufwies, sich also aufgrund ihrere schlechteren Ausgangswerte leichter hätte verbessern können. Die Patienten der PMR-Gruppe profitieren demnach schneller von ihrer Therapie, während die Teilnehmer der IPT-I- Gruppe sich möglicherweise erst durch die Therapie ihrer noch zu bearbeitenden interpersonellen Probleme bewusst werden, darüber vermehrt grübeln und deshalb ihren Schlaf subjektiv nicht signifikant verbessern können (Paterok 1993). Die Auswertung des Fragebogens SF-B in der vorliegenden Untersuchung bringt somit keine neuen Erkenntnisse über die qualitativen Parameter eines zweiwöchigen 
Zeitraumes gegenüber Müller-Popkes 1997. Nach wie vor ist somit auch unter Berücksichtigung aller zur Verfügung stehenden Daten die PMR der IPT-I, deren Gruppe sogar vor Therapiebeginn eine signifikant schlechtere Schlafqualität aufwies und sich daher leichter hätte verbessern können, überlegen.

\subsubsection{Der Schlaf in der Bewertung der vergangenen Nacht}

Vor allem in den Fragebögen SF-A, die sich jeweils nur auf die vergangene Nacht beziehen, waren Effekte sowohl in der Qualität als auch in der Quantität zu erwarten gewesen. Die sich in der Varianzanalyse im Vergleich des Schlafes vor mit dem Schlaf nach der Behandlung darstellenden Therapieeffekte werden zuerst hinsichtlich quantitativer, dann qualitativer Aspekte diskutiert. Die Ergebnisse des SF-AFragebogens sind mit Vorbehalt zu betrachten, da die Daten dafür lediglich zu den nicht repräsentativen Zeitpunkten der Eingangs- und Kontrollpolysomnografietermine erhoben wurden. Hierauf wird in Kapitel 4.4.4. noch näher eingegangen.

\subsubsection{1 quantitative Parameter}

Im SF-A zeigt sich im Gegensatz zum Prä-Post-Vergleich des VIS-M (Müller-Popkes 1997) keine Besserung der TST in der PMR-Gruppe, sondern eine, wenn auch statistisch insignifikante, Verschlechterung. Die Ursache für dieses unterschiedliche Ergebnis ist, dass der SF-A im Gegensatz zum VIS-M nur zu den zwei Messzeitpunkten vor und nach der Behandlung eingesetzt worden war. Zum einen hat sich bis zum Beginn der Behandlung, also bis zur Eingangspolysomnografie, dem Ausgangsmesspunkt des SF-A, die PMR-Gruppe bereits ohne spezifische Therapie derartig verbessern können (Müller-Popkes 1997), dass eine weitere Verbesserung schwerlich möglich war. Zum anderen fehlen im SF-A Messungen zu Katamnesezeitpunkten. Auch in der Darstellung der VIS-Ergebnisse hat Müller-Popkes 1997 auf Seite 139 zu den zwei Messzeitpunkten vor und nach der Behandlung, an denen der SF-A eingesetzt wurde, eine Verschlechterung dokumentiert, die jedoch durch die statistische Gesamtberechnung unter Einschluss der weiteren drei Messzeitpunkte Baseline, Drei- und Sechsmonatskatamnese (Müller-Popkes 1997) nicht ins Gewicht fiel. Große Auswirkungen auf die TST waren jedoch ohnehin nicht zu erwarten gewesen. Auch Espie et al. (1989) haben zu Behandlungsende nur eine knapp elfprozentige Verbesserung der TST erreicht. Morin et al. (1994) konnten in ihrer MetaAnalyse über sieben Behandlungsbedingungen ähnlich leichte Erfolge zu Behandlungsende feststellen. Die IPT-I-Gruppe zeigte eine insignifikante Verbesserung der TST von Therapiebeginn bis zum Behandlungsende, die auch in diesem Zeitraum in 
der zuvor durchgeführten Untersuchung des VIS-M (Müller-Popkes 1997, S. 139) gezeigt werden konnte.

Die nächtliche Wachzeit (WASO), zu der bisher noch keine Daten veröffentlicht worden sind, veränderte sich im SF-A im Sinne einer Besserung in der IPT-I-Gruppe von 45 auf 31 min und einer Verschlechterung in der PMR-Gruppe von 35 auf 47 min. Diese Änderungen um immerhin ein Drittel bzw. ein Viertel sind jedoch nicht signifikant. Eine der Ursachen für die gegensätzliche Entwicklung der beiden Gruppen können die unterschiedlichen Ausgangswerte zu Beginn der eigentlichen Behandlung sein. Bis zu diesem Zeitpunkt hatte sich die IPT-I verschlechtert und die PMR verbessert, obwohl zuvor nur ein Eingangsgespräch über die jeweilige zu erwartende Therapie stattgefunden hatte. Die Gründe für die Verschlechterung der IPT-I-Gruppe nach dem Eingangsgespräch wurden bereits unter 4.4.1.1.2 aufgeführt, die Verbesserung der PMR-Gruppe wurde bereits diskutiert und auf „Vorschusslorbeeren“ (MüllerPopkes 1997, S. 170), die die Patienten der Therapie bereits vor Behandlungsbeginn gaben, sowie auf kognitive Umstrukturierung zurückgeführt.

Die Häufigkeit der Schlafunterbrechungen (HdSU) im SF-A, zu der bisher ebenfalls noch keine Daten veröffentlicht sind, besserte sich nur in der IPT-I-Gruppe, jedoch nicht signifikant. In den Auswirkungen der Behandlungen auf die Durchschlafstörung zeigt sich somit die IPT-I gegenüber der PMR leicht überlegen, auch wenn hier keine Signifikanz der Verbesserungen erzielt werden kann und man bedenken muss, dass sich angesichts schlechterer Ausgangswerte der IPT-I mehr Möglichkeit zu einer Verbesserung bietet. Die wenn auch insignifikante Verbesserung der Häufigkeit der Schlafunterbrechungen in der IPT-I-Gruppe ist ein bedeutsames Ergebnis.

Die Einschlaflatenzen zeigen in der PMR-Gruppe bei mäßig gutem Ausgangwert eine Verschlechterung um eine Medianklasse. Ein Grund hierfür könnte die Anwendung der Übungen sein. Wir wissen nicht, ob die PMR-Patienten, die während der Therapie dazu angehalten worden waren, ihre Übungen auch vor dem Einschlafen durchzuführen, den Zeitbedarf hierfür in der subjektiven Beurteilung auf die Einschlafzeit aufgeschlagen haben, oder ob sie in den Kontrollnächten auf die Übungen verzichtet haben und dieser Verzicht aufgrund der fehlenden einschlaffördernden Entspannungsübung zu einer längeren subjektiven Einschlafzeit geführt hat. Freedman und Papsdorf (1976) hatten ihren Patienten 20 Minuten zum Üben zur Verfügung gestellt, die nicht mit zur Einschlafzeit gerechnet wurden, in der dieser Untersuchung zugrunde liegenden Studie (Müller-Popkes 1997) fand die Übungszeit jedoch keine Berücksichtigung. In dem Schlaffragebogen SF-A wird nach der Zeit des Lichtlöschens und nach der Dauer bis 
zum Einschlafen gefragt sowie nach den Gründen, warum man nicht einschlafen konnte. Somit hätte durch ein korrektes Ausfüllen des Bogens bei Durchführung der einschlaffördernden Entspannungsübungen im Dunkeln eine falsch hohe Einschlafzeit resultieren können (s. Kapitel 4.4.2.2). In der IPT-I-Gruppe ist die signifikante Verringerung der Einschlaflatenz um eine Medianklasse ein positives Ergebnis, das dem der VIS-M-Fragebögen (Müller-Popkes 1997, S. 138f) entspricht. Durch das strenge Auswertungsverfahren mittels der Bonferoni-Adjustierung ging die Signifikanz in der vorliegenden Untersuchung jedoch verloren.

Zusammenfassend zeigt die Betrachtung der subjektiven Schlafquantität im täglichen Rückblick in der vorliegenden Untersuchung keine signifikanten Verbesserung beider Gruppen, die positive Auswirkung der IPT-I auf die Häufigkeit der Schlafunterbrechungen ist jedoch ein bedeutsamer neuer Aspekt, der auf geringere psychische Belastung zurückzuführen sein könnte. Aus dem Vergleich beider Untersuchungen, der vorliegenden sowie der von Müller-Popkes 1997, zugrundeliegenden Daten und Messzeitpunkte wird folgendes deutlich: Durch das Ausmaß zuvor unerwarteter nicht therapiespezifischer prätherapeutischer Veränderungen von der Baseline bis zum Behandlungsbeginn (Müller-Popkes 1997) stellen sich Art, Ausmaß und Signifikanz der Behandlungseffekte unterschiedlich dar.

\subsubsection{2 qualitative Parameter}

Die fast signifikante Verbesserung der Schlafqualität von Therapiebeginn bis zum Behandlungsende im SF-A ist in der IPT-I-Gruppe möglicherweise darauf zurückzuführen, dass die Patienten durch die Therapie tagsüber ihre zwischenmenschlichen Kontakte befriedigender gestalten können und daher auch ihren Schlaf als gebessert empfinden (Coates et al. 1983). Die insignifikante Verschlechterung in der PMR-Gruppe in dem gleichen Zeitraum wurde auch bei Müller-Popkes 1997 beobachtet, dort wurde der sehr gute prätherapeutische Ausgangswert durch „kognitive Umstrukturierung, sowie durch die Vergabe von „Vorschusslorbeeren“ an die Behandlung, zit. nach Müller-Popkes 1997, S. 170 erklärt. Die Veränderungen des morgendlichen Erholungsgefühls sind geringer, jedoch von gleicher Qualität wie bei der Schlafqualität, möglicherweise aus den entsprechenden Gründen. 


\subsection{2 objektive Schlafparameter}

\subsubsection{Die Auswertung polysomnografischer Ableitungen}

Die objektiven Ergebnisse sind in beiden Gruppen durch die Verfasserin der vorliegenden Untersuchung ermittelt worden, daher miteinander vergleichbar, denn von Auswerter zu Auswerter gibt es eine hohe Variabilität bezüglich der Auswertung nach Rechtschaffen und Kales durch die individuelle Interpretation der Regeln durch den jeweiligen Auswerter (Collop 2002). Die Verfasserin der vorliegenden Untersuchung ist über mehrere Monate intensiv in die Auswertung eingearbeitet worden und in der Befundung nach Rechtschaffen und Kales intensiv betreut und supervidiert worden, was von Chediak et al. 2006 als ausreichend befunden wird. Die Auswertung nach Rechtschaffen und Kales (1968) ist jedoch bei Fragmentation des Schlafes weniger zuverlässig (Norman et al. 2001), und die dynamische Struktur des Schlafes wird damit nicht adaquat erfasst (Nieuwenhuijs 2006).

Die Ergebnisse der sowohl vor als auch nach der Behandlung für beide aufeinanderfolgende Nächte gemittelten objektiven Schlafparameter sind bereits von Müller-Popkes 1997 auf den Seiten 127-129 statistisch ausgewertet und kommentiert und auf den Seiten 167-169 ausführlich diskutiert worden.

Hochpathologische Ausgangswerte mit Raum für deutliche Verbesserungen konnten nicht erwartete werden, denn die meisten Insomniepatienten schlafen in weniger als 30 Minuten ein und länger als 6,5 Stunden (Carskadon et al. 1976). Nach den vorliegenden Kriterien (Williams et al. 1974) waren denn auch die Einschlaflatenz unauffällig, die übrigen Parameter tatsächliche Schlafzeit, Schlafeffizienz unterdurchschnittlich und die nächtliche Wachzeit überdurchschnittlich, insgesamt jedoch nicht sehr auffällig. (Müller-Popkes 1997). Bei strenger Hypothesenprüfung konnten keine signifikanten Verbesserungen in allen vier Schlafparametern erzielt werden, es zeigten sich jedoch positive Tendenzen in den Parametern tatsächliche Schlafzeit und nächtliche Wachzeit, in der IPT-I-Gruppe sogar bei der Schlafeffizienz (Müller-Popkes 1997). Die im Vergleich längere Einschlaflatenz in der Kontrollpolysomnografie bei den PMRPatienten ließe sich möglicherweise damit erklären, dass sich in dieser Gruppe durch die Durchführung der Übungen vor dem Einschlafen die Einschlaflatenz verlängert hat, dies wäre ein systematischer Fehler.

Auch die fehlende Signifikanz der Verbesserungen der tatsächlichen Schlafzeit muss vor dem Hintergrund der normalen Gesamtschlafzeit gesehen werden: posttherapeutisch reicht die IPT-I-Gruppe an die 390-Minuten-Grenze (Bixler et al. 1973, ASDC 1979) 
knapp heran und wird in der PMR-Gruppe sogar überschritten. Die nächtliche Gesamtwachzeit war in beiden Gruppen nach Behandlungsende noch deutlich erhöht. Hier haben sich beide Gruppen nur leicht verbessern können. Wenn auch nach bisherigem Forschungsstand für die PMR-Gruppe ein besseres Ergebnis nicht unbedingt erwartet werden konnte, so ist die statistisch insignifikante Verbesserung um lediglich ein Drittel in der IPT-I-Gruppe enttäuschend. Beim Blick in die einzelnen Nächte fällt jedoch besonders der Unterschied der nächtlichen Gesamtwachzeit zwischen der ersten und zweiten Kontrollnacht auf: ein IPT-I-Gruppenmittelwert von 67 Minuten in der ersten Nacht der Kontrollpolysomnografie steht einem fast halb so hoher Wert von 39 Minuten in der darauffolgenden Nacht gegenüber, diese Unterschiede sind in der PMRGruppe mit 53 bzw. 45 Minuten deutlich geringer. Diese erhebliche Schwankung erklärt sich durch die geringe Stichprobengröße: Die schlechten Werte Einzelner führen bereits zu einer deutlichen Veränderung des Gruppenmittelwertes. Der Erklärungsversuch, die in der PMR-Gruppe im Verhältnis zur IPT-I-Gruppe deutlich geringere Schwankung zwischen den Nächten als Therapieerfolg im Sinne einer Besserung des Erstnachteffektes durch die Durchführung der Entspannungsübungen zu werten, ist rein spekulativ.

Die schlechte Schlafeffizienz der IPT-I-Gruppe in der Eingangspolysomnografie ist vor dem Hintergrund einer Verschlechterung des subjektiven Schlafes von der Baseline bis zur Eingangspolysomnografie zu interpretieren. Durch diese „prätherapeutische“ Verschlechterung, die in Kapitel 4.4.3 noch ausführlich diskutiert werden wird, ist von einer tatsächlich geringeren Verbesserung auszugehen. Die posttherapeutischen Schlafeffizienzen beider Gruppen liegen mit 84 bzw. 82,9 nur geringfügig unter der von Frankel et al. (1976) zur Unterscheidung zwischen Insomnikern und Nichtinsomnikern postulierten 85\%-Marke. Demnach wäre eine weitere Besserung kaum zu erreichen gewesen. Coates et al. (1982) setzten in ihrer Studie eine Schlafeffizienz von 88\% zur Differenzierung von guten und schlechten Schläfern als ein Kriterium an.

Weitere polysomnografische Aspekte wie REM-Schlaf und die Mikrostruktur des Schlafes sind in dieser Untersuchung unberücksichtigt geblieben, um die Zahl der Variablen auf ein statistisch verwertbares Maß zu begrenzen.

Aus finanziellen und personellen Gründen war nur ein zweitägiger polysomnografischer Kontrolltermin möglich. Seine Aussagefähigkeit über die klinische Signifikanz der Behandlungen wäre möglicherweise größer gewesen, hätte er statt zum Therapieende zum Zeitpunkt der Drei- oder Sechsmonatskatamnese stattgefunden (Paterok 1993), da allein eine über das Therapieende hinaus anhaltende oder sogar weiter zunehmende 
Verbesserung des Schlafes klinisch relevant ist. Etwaige Verbesserungen könnten somit dem Patienten allein und nicht dem Therapeuten zugeschrieben werden, was das Kompetenzgefühl des Patienten verbessern würde.

Zusammenfassend lässt sich das geringe Ausmaß der Verbesserungen des objektiven Schlafes mit den vorliegenden polysomnografisch kontrollierten Studien der PMR (Freedman und Papsdorf 1976, Borkovec und Weerts 1976) in Einklang bringen (Müller-Popkes 1997). Die Auswirkung der IPT-I auf die Schlafeffizienz bedarf einer weiteren Überprüfung bei modifiziertem Studiendesign, da, wie bereits in diesem Unterkapitel ausführlich erläutert, durch die gegensätzliche prätherapeutische Veränderung in beiden Gruppen die erreichte statistische Signifikanz relativiert werden muss. Angesichts der Schlafvariabilität von Insomnikern kann es sein, dass sowohl die Schlafstörung selbst (Trinder 1988) als auch Behandlungseffekte (Borkovec und Weerts 1976) in einer zweinächtlichen Schlafableitung nicht abgebildet wurden. Zumindest bei älteren Patienten wäre für die Abbildung der Einschlaflatenz eine polysomnografische Aufzeichnung von mehr als zwei Wochen notwendig (Wohlgemuth et al. 1999). Ein entsprechendes Studiendesign wäre aus personellen und finanziellen Gründen schwer zu realisieren. Etwaige mikrostrukturelle Schlafveränderungen, die die Nichtwahrnehmung des Schlafzustandes erklären könnten, fanden in die Auswertung nach Rechtschaffen und Kales 1968 keinen Eingang. Die Heterogenität der Stichprobe in der vorliegenden Untersuchung ist neben der Schlafvariabilität und der Auswertungskriterien ein weiterer Grund für die fehlende Abbildung möglicher Therapieeffekte, da sich Ein- und Durchschlafstörung gegenseitig aufheben und bei Patienten mit bereits prätherapeutisch kaum verminderten objektiven Schlafparametern eine weitere Steigerung schwerlich möglich ist.

\subsubsection{Gegenüberstellung von objektiven und subjektiven Schlafparametern vor und} nach der Behandlung

+Aus der Gegenüberstellung subjektiver und objektiver Daten kann spekuliert werden, dass in der zu hoch eingeschätzten Einschlaflatenz nach Behandlungsende die PMRÜbungszeit enthalten sein könnte. Es wird im SF-A- und SF-B-Fragebogen nach der Zeit nach dem Lichtlöschen bis zum Einschlafen gefragt, wenn also im Dunkeln die Übungen durchgeführt werden, sind sie möglicherweise, je nach der Auslegung durch den Studienteilnehmer, in der Einschlaflatenz mit enthalten. Somit hätte durch ein korrektes Ausfüllen des Bogens bei Durchführung der einschlaffördernden Entspannungsübungen im Dunkeln eine falsch hohe Einschlafzeit resultieren können. 
Ihre tatsächliche Schlafzeit hat die PMR-Gruppe zur Eingangspolysomnografie fast korrekt eingeschätzt, sich jedoch zur Kontrollpolysomnografie um ca. 40-50 Minuten verschätzt. Diese zu niedrig eingeschätzte tatsächliche Schlafzeit in der PMR-Gruppe nach Behandlungsende kann nicht durch die Übungszeit bedingt sein, da danach in den SF-A/B-Bögen direkt gefragt wurde. Dass die nächtliche Wachzeit in der mittelfristigen Beurteilung eher länger, in der täglichen eher kürzer eingeschätzt wird als die objektive nächtliche Wachzeit, deckt sich mit der Literatur (Mendelson 1987). Ein direkter Vergleich kann zwischen subjektiven und objektiven Daten nicht angestellt werden, da der SF-A-Wert ein Gruppenmittelwert aus erster und zweiter Eingangsbeziehungsweise erster und zweiter Kontrollpolysomnografienacht ist, der PSG-Wert dagegen der Gruppenmittelwert einer einzelnen Nacht. Die Patienten waren schon vor Therapiebeginn nicht schwer schlafgestört, wie sich in der polysomnografischen Eingangsuntersuchung zeigte (Müller-Popkes 1997). Da die subjektive Schlafbewertung im Schlaflabor deutlich von der subjektiven Schlafbewertung einer Nacht in häuslicher Umgebung abwich, ist die Aussagekraft der objektiven Parameter zu relativieren. Die Grafiken der Verlaufsbetrachtung verdeutlichen das Abweichen der Schlaflabornächte (s. Kapitel 3.6), hierauf wird im Verlauf der Diskussion noch näher eingegangen. Man kann davon ausgehen, dass die Patienten im Schlaflabor in den jeweils zwei Nächten nach und insbesondere vor der Behandlung einfach nicht so schliefen wie zu Hause. Ambulante Polysomnografien in gewohnter häuslicher Umgebung (Coates et al. 1982) hätten einen weniger verzerrten Blick auf den objektiven Schlaf erlaubt.

\subsubsection{Verlaufsbetrachtung anhand subjektiver Schlafparameter (VIS-M)}

Für die Verlaufsbetrachtung wurde jeweils aus 5 Fragebögen zwischen den wöchentlichen Therapiesitzungen ein Mittelwert berechnet. Die Schwankungen, die sich von Woche zu Woche im Verlauf der Therapie ergeben, sind insgesamt nicht sehr hoch. In der vorliegenden Untersuchung wurde der Verlauf während der Behandlung, das heißt von der ersten eigentlichen Therapiesitzung bis zur letzten Sitzung berücksichtigt. Die zu der Baseline und zu den Eingangs- und Kontrollpolysomnografieterminen erhobenen Daten wurden zunächst nicht berücksichtigt, da zum einen keine Mittelwertbildung aus fünf Messungen erfolgen konnte, zum anderen die bereits bei Müller-Popkes 1997 nachgewiesenen Veränderungen von der Baseline bis zum Eingangspolysomnografietermin offensichtlich unspezifischen Effekten zugrunde liegen: In diesem Zeitraum erfolgte keine spezifische Behandlung, die Patienten der IPT-I-Gruppe waren jedoch bereits in der ersten Sitzung für einen möglichen Zusammenhang zwischen ihrer Schlafstörung und interpersonellen Problemen 
sensibilisiert worden. Da diese Sensibilisierung in der PMR-Gruppe wegen hier fehlender Thematisierung des Zusammenhangs von Schlafstörung und interpersonellen Problemen nicht stattfinden konnte, sind die Effekte vor Behandlungsbeginn nicht so unspezifisch wie vermutet. Darüberhinaus hatte sich in der Datensichtung für die vorliegende Untersuchung gezeigt, dass sich die Eingangs- und Kontrollpolysomnografietermine nicht gut in den übrigen Verlauf integrieren ließen, die Patienten demnach im Schlaflabor ihren Schlaf entweder nicht so beurteilten wie zu Hause oder im Schlaflabor nicht so schliefen wie zu Hause.

\subsubsection{Quantitative Parameter}

Die zu Beginn in der IPT-I-Gruppe im Mittel über sechs Stunden liegende tatsächliche Schlafzeit (TST) fiel nur vorübergehend in den mittleren Sitzungen um eine knappe halbe Stunde ab, konnte unter leichten Schwankungen gehalten werden und fiel erst zur letzten Therapiesitzung erneut um eine gute halbe Stunde ab, möglicherweise erklärt durch das Abschiedsphänomen: Durch das bevorstehende Behandlungsende könnten die Patienten befürchten, zukünftig die Sitzungen zu vermissen; eine daraus entstehende erhöhte psychische Belastung könnte sich negativ auf den Schlaf ausgewirkt haben. Die PMR-Gruppe konnte sich während der Therapie von einem leicht unter der IPT-IGruppe liegenden Ausgangswert verbessern und diese Verbesserung auch teilweise halten. Eine signifikante Veränderung war für diese Gruppe bereits festgestellt worden (Müller-Popkes 1997). Sie entspricht ihrem Ausmaß den Angaben in der Literatur: Espie et al. (1989) haben zu Behandlungsende eine knapp elfprozentige Verbesserung der TST erreicht. Die Schwankungen in der Gesamtschlafzeit der IPT-I-Gruppe mit tendenzieller Verschlechterung in den mittleren Phasen sind möglicherweise auf der Problembearbeitung in den mittleren Sitzungen zurückzuführen, bei der sich die hierdurch entstehende psychische Belastung negativ auf den Schlaf auswirkt. Ein Zusammenhang zwischen psychologischem Stress und erhöhtem physiologischen Arousal während des Non-REM-Schlafes bei Patienten mit primärer Insomnie konnte von Hall et al. 2007 nachgewiesen werden.

Die Schwankungen der Einschlaflatenz (EL) unter der Therapie betragen ca. 10 Minuten und sind bei beiden Gruppen von ähnlichem Ausmaß (Abb. 3.10). Im Rahmen dieser Schwankungen kommt es zu leichten Verbesserungen der IPT-I, während die PMR sich leicht verschlechtert. Wie bei der Interpretation der Daten des SF-A stellt sich auch hier die Frage, ob die PMR-Patienten, die während der Therapie dazu angehalten worden waren, ihre Übungen vor dem Einschlafen durchzuführen, die hierfür benötigte Zeit in der subjektiven Beurteilung auf die Einschlafzeit aufgeschlagen haben. 
Die Schwankungen der Mittelwerte der quantitativen Schlafparameter im Verlauf sind insgesamt moderat. Bei der IPT-I-Gruppen liegt die größte Schwankung in der tatsächlichen Schlafzeit bei einer guten halben Stunde, in der PMR-Gruppe unter einer dreiviertel Stunde, in der Einschlaflatenz liegen sie bei ca. einer Viertelstunde.

Interessant wäre die Betrachtung der Nacht-zu-Nacht-Variabilität gewesen, die durch die Verlaufsbetrachtung anhand der täglich ausgefüllten visuellen Analogskalen (VISM) möglich wäre und Gegenstand weiterer Untersuchungen sein könnte. Dieser Parameter ist bei Schlafgestörten sehr ausgeprägt (Hauri 1989), er kann für den Leidensdruck eines Schlafgestörten von erheblicher Bedeutung (Espie et al. 1989) und mitunter wichtiger als die tatsächliche Schlafzeit (Coates et al. 1982) sein, weil er dem Patienten das Gefühl des Ausgeliefertseins und der Unbeherrschbarkeit vermittelt. Dieser Parameter ist jedoch bisher noch in keiner Studie zur Überprüfung des Therapieerfolges eingesetzt worden. Eine Überprüfung der objektiven Nacht-zu-NachtVariabilität wäre mittels ambulanter Polysomnografie möglich (Coates et al. 1982), die subjektive Schlafqualität könnte zum Beispiel mit visuellen Analogskalen zur Schwankungsbreite des Schlafes oder mit entsprechenden Fragen ermittelt werden, die auf die Unvorhersehbarkeit „schlechter“ Nächte und den damit verbundenen Leidensdruck abzielen.

\subsubsection{Qualitative Parameter}

Die im mittleren Bereich liegende Schlafqualität (SQ) zeigt im Verlauf der elf Messzeitpunkte unter zunehmenden diskreten Schwankungen gegen Ende der Behandlung in der PMR-Gruppe eine leichte Verbesserung, die auf die zunehmende Wirkung des Entspannungsverfahrens zurückgeführt werden kann. Die tendenzielle Verschlechterung in der IPT-I-Gruppe zeigt zunehmende Schwankungen, die eine diskrete Besserungstendenz zum Behandlungsende hin erkennen lassen. Diese Tendenz kann sich, möglicherweise bedingt durch das Abschiedsphänomen (s. Kapitel 4.4.3.1), nicht durchsetzen. Die diskrete Verschlechterung der Schlafqualität im mittleren Behandlungsabschnitt könnte auf die therapiespezifische Bearbeitung der interpersonellen Problembereiche zurückgeführt werden. Es wäre sehr spekulativ, die zunehmenden Schwankungen im letzten Behandlungsdrittel therapiespezifisch durch die zunehmenden Umsetzungsversuche der psychotherapeutischen Arbeit an den interpersonellen Problembereichen erklären zu wollen, wahrscheinlich handelt es sich bei der sehr kleinen Stichprobe dagegen einfach um zufällige Schwankungen. 
Das morgendliche Erholungsgefühl (mE) im Verlauf der dargestellten elf Messzeitpunkte wird von der IPT-I-Gruppe durchgehend als schlechter beurteilt als von der PMR-Gruppe, dabei zeigt sich zu allen Messzeitpunkten weder für die IPT-I- noch für die PMR-Gruppe eine Verbesserung. Eine vorübergehende Verschlechterung in der IPT-I-Gruppe während des mittleren Therapieabschnitts könnte auch bei diesem Parameter auf die Bearbeitung des Problembereichs zurückzuführen sein.

Im direkten Anschluss erfolgt die Diskussion der Einbettung des Verlaufs in die bereits von Müller-Popkes 1997 veröffentlichten Rahmendaten.

\subsubsection{Verlaufsbetrachtung anhand subjektiver Schlafparameter (VIS-M) mit Berücksichtigung der Baseline und der Daten aus dem Schlaflabor}

Die vorliegende Untersuchung zeigt, dass die subjektiven Messwerte des VIS-MFragebogens zu den Zeitpunkten der Eingangs- und Kontrollpolysomnografien aus den Verlaufskurven zum Teil deutlich herausfallen. Somit sind auch die Ergebnisse des SFA-Fragebogens mit Vorbehalt zu betrachten, da die zugrunde liegenden Daten lediglich zu eben diesen nicht repräsentativen Zeitpunkten erhoben wurden.

Die Verlaufsbetrachtung über alle 14 Messzeitpunkte inklusive der zum Teil von Müller-Popkes 1997 bereits veröffentlichten Daten der Baseline, der Eingangs- und der Kontrollpolysomnografie zeigt folgendes: Durch den Vergleich der Ergebnisse zum Zeitpunkt von Eingangs- und Kontrollpolysomnografie lassen sich Rückschlüsse auf die Wirksamkeit der beiden zu vergleichenden Behandlungsformen allenfalls eingeschränkt ziehen, denn: Bei beiden Gruppen lassen sich bereits vor Beginn der eigentlichen Behandlung in der Eingangspolysomnografie deutliche Veränderungen erkennen. Einige der im Schlaflabor ermittelten Daten weichen deutlich von den in häuslicher Umgebung erhobenen Daten der Verlaufsbetrachtung ab und verzerren bei einigen Parametern das Bild. Die Veränderungen von der Baseline bis zur Eingangspolysomnografie werden zunächst beschrieben:

Die Einschlaflatenz (EL) verschlechterte sich in der IPT-I-Gruppe von der Baseline bis zur Eingangspolysomnografie deutlich, die tatsächliche Schlafzeit (TST), die Schlafqualität (SQ) und das morgendliche Erholungsgefühl (mE) verschlechterten sich leicht. Der randomisierten Zuteilung der Patienten zu den Untersuchungsgruppen war ein Erstgespräch vorausgegangen, das die unterschiedliche Ausprägung der Schlafstörung bewirkt haben könnte. Darin waren die IPT-I-Patienten bereits mit der Möglichkeit konfrontiert worden, dass zwischen ihrer Schlafstörung und ihren interpersonellen Problemen ein Zusammenhang bestehen könnte. Die im Vergleich zur 
IPT-Gruppe gegensätzlichen Veränderungen in der PMR-Gruppe sind noch bemerkenswerter. Alle vier untersuchten Parameter TST, EL, SQ und mE besserten sich deutlich, wie die Verlaufsbetrachtung der Ergebnisse zeigt. Schon allein die Aussicht auf das Erlernen einer bewährten Entspannungstherapie hatte eine positive Wirkung (Müller-Popkes 1997).

Im Anschluss an die Diskussion der Veränderungen von der Baseline bis zur Eingangspolysomnografie erfolgt nun die Diskussion der Veränderungen im Verlauf der Behandlung, eingebettet in die Rahmendaten der Baseline und der Messungen im Schlaflabor.

Vor dem eigentlichen Behandlungsbeginn verbessert sich die tatsächliche Schlafzeit in beiden Gruppen von der Baseline bis zum Behandlungsbeginn nach der Eingangspolysomnografie um mehr als 20 Minuten, obwohl bis dahin noch keine spezifische Therapie, entweder PMR oder IPT-I begonnen worden war. Nach der randomisierten Zuteilung war mit den Patienten lediglich ein Eingangsgespräch mit Anamneseerhebung und Informationsvermittlung über den geplanten Studienablauf geführt worden. Hierbei könnte jedoch die IPT-I-Gruppe durch die bereits auf die spezifische Therapie ausgerichtete Anamneseerhebung für einen möglichen Zusammenhang zwischen Schlafstörung und diese auslösende interpersonelle Probleme sensibilisiert worden sein, was sich durch vermehrtes Grübeln nachteilig auf den Schlaf in der Eingangspolysomnografie ausgewirkt haben könnte, da die Beschäftigung mit eigenen Problemen zu vermehrtem Grübeln und dadurch zu einer Verschlechterung geführt haben könnte (Paterok 1993). Die Erwartung einer aufdeckenden Behandlung könnte zudem eine andere, angstauslösende Wirkung haben als die Aussicht auf eine reine Entspannungstherapie (Müller-Popkes 1997).

Die PMR-Gruppe verbessert ihre tatsächliche Schlafzeit vor Therapiebeginn deutlich (s. Abb. 3.13-3.16). Auf diesen guten Wert pendelt sich die PMR-Gruppe erst in der zweiten Behandlungshälfte wieder ein, kann sich bezüglich der TST auch noch weiter verbessern, zeigt jedoch während der abschließenden Kontrollpolysomnografie eine deutlich kürzere tatsächliche Schlafzeit, die fast auf das Baseline-Niveau abgesunken ist. Die Ursache könnte das Abschiedsphänomen (s. Kapitel 4.4.3.1) sein.

Die Einschlaflatenz bessert sich in der IPT-I-Gruppe im Gegensatz zur PMR-Gruppe, letztere hätte unter Berücksichtigung der Baseline (Müller-Popkes 1997) auch eine Besserung erlangt, wenn nur die Werte bis zur vorletzten Sitzung berücksichtigt worden wären, denn vor der letzten Sitzung und in der Kontrollpolysomnografie verschlechtert 
sich die PMR-Gruppe wieder, möglicherweise durch das Abschiedsphänomen (s. Kapitel 4.4.3.1); auch das Eingehen der Übungszeit in die Einschlaflatenz, wie ebenfalls in Kapitel 4.4.3.1 diskutiert, wäre als Erklärung für die Verschlechterung denkbar.

In der Schlafqualität kommt es im Wesentlichen in beiden Gruppen zu zunehmenden, wenn auch geringen Schwankungen. In der PMR-Gruppe zeigt sich eine kontinuierliche Verbesserung bis zum letzten Behandlungstermin mit anschließendem Einbruch in der Kontrollpolysomnografie, die sowohl mit dem Abschiedsphänomen zu erklären wäre als auch mit der Tatsache, dass die PMR-Patienten in der fremden Umgebung des Schlaflabors doch einfach nicht so gut schlafen wie zu Hause. Das Phänomen der guten Eingangspolysomnografie ist bereits zuvor in diesem Unterkapitel erklärt worden, ebenso die diskrete Verschlechterung der IPT-I-Gruppe in der Eingangspolysomnografie. Die IPT-I-Gruppe zeigt im Vergleich zur Baseline im Wesentlichen eine diskrete Verbesserungstendenz.

Das morgendliche Erholungsgefühl verbessert sich nicht in der IPT-I-Gruppe, unter Berücksichtigung der Baseline (Müller-Popkes 1997) in der PMR-Gruppe jedoch leicht. Dieses für beide Gruppen unterschiedliche Verhalten in der täglichen Einschätzung des morgendlichen Erholungsgefühls wäre ohne die Verlaufsbetrachtung, in der sich während der gesamten Behandlung die PMR-Gruppe durchgehend morgens besser erholt zeigt als die IPT-I-Gruppe, nicht deutlich geworden. Der positive Effekt der PMR auf das morgendliche Erholungsgefühl überrascht nicht (Turner und Ascher 1979), ist jedoch auch schon stärker beobachtet worden (Espie et al. 1989). In der vorliegenden Untersuchung konnte die Verbesserung in der PMR-Gruppe während der laufenden Behandlung ohne Berücksichtigung der Baseline nicht dargestellt werden (s. Kapitel 4.4.3). Die nun in diesem Unterkapitel diskutierten Nachuntersuchungen mit Einbettung der Verlaufsbetrachtung in die Daten Baseline, Eingangs- und Kontrollpolysomnografie verdeutlichen auch am Beispiel des morgendlichen Erholungsgefühls, dass „unspezifische“ Effekte Einfluss auf die Ergebnisse haben, vor allem bei kleinen Stichproben wie in der zugrunde liegenden Studie.

Eine Veränderung des Studiendesigns könnte die prätherapeutischen Veränderungen verhindern und sich folgendermaßen gestalten: Ein vor der Eingangspolysomnografie stattfindendes Erstgespräch müsste die Patienten vor der Zuweisung zu den jeweiligen Behandlungsgruppen über beide Therapien gleichermaßen aufklären. Erst nach der dann folgenden Polysomnografie und dem Ausfüllen der begleitenden Fragebögen dürfte die randomisierte Gruppenzuteilung erfolgen. Da in der praktischen Durchführung aus Kapazitätsgründen die Patienten nicht parallel, sondern zeitlich versetzt 
polysomnografiert und behandelt wurden, war eine doppelblind durchgeführte Eingangspolysomnografie praktisch undurchführbar. Unter Verzicht auf jegliche Parallelisierung wäre eine postpolysomnografische randomisierte Zuteilung ausreichend, um die in unserer Studie beobachteten gegensätzlichen Veränderungen der beiden Gruppen von der Baseline bis zum Zeitpunkt der Eingangspolysomnografie abzuschwächen. Eine dann mögliche Unausgewogenheit in der Geschlechtsverteilung brächte wiederum Probleme in der Auswertung wegen eingeschränkter Vergleichbarkeit mit sich.

\subsubsection{Veränderungen in den ersten zwei Dritteln des Behandlungszeitraums}

Um zu klären, ob bereits acht Sitzungen IPT-I zu einer Verbesserung des Schlafs führen können, waren die Messzeiträume der zweiwöchigen Baseline sowie der dem zweiten und neunten Sitzungstermin jeweils vorhergehenden fünf Tage betrachtet worden. Durch diese Auswahl sollte das gegensätzliche Verhalten der Gruppen in den Eingangspolysomnografienächten unberücksichtigt gelassen werden, dem die bereits im vorangegangenen Unterkapitel diskutierten Effekte zugrunde lagen. Die IPT-I-Gruppe verbesserte sich während der spezifischen Therapie vom Zeitraum vor dem zweiten bis zum Zeitraum vor dem neunten Sitzungstermin in der Schlafqualität (SQ) nicht. Das morgendliche Erholungsgefühl ( $\mathrm{mE}$ ) änderte sich durch die ersten acht Sitzungen in der IPT-I-Gruppe gar nicht, in der PMR-Gruppe nur tendenziell während des unspezifischen Anteiles der Therapie, diese prätherapeutische Verbesserung in der PMR-Gruppe blieb während der Sitzungen erhalten.

Bei der vorliegenden Untersuchung wurde aufgrund der fehlenden Einbeziehung der letzten Sitzungen ein mögliches Abschiedsphänomen (s. Kapitel 4.4.3.1) nicht mit erfasst. Für ein solches Phänomen gibt es jedoch bei der Verlaufsbetrachtung über die elf Messzeiträume - vor der 2. Sitzung bis vor der 12. und damit letzten Sitzung Hinweise. Eine Verkürzung der IPT-I würde die Thematisierung des bevorstehenden Therapieendes aus Zeitgründen erschweren, was möglicherweise einen negativen Effekt auf den Therapieerfolg hätte.

Auch die quantitativen Parameter sollten varianzanalytisch im Verlauf betrachtet werden, ohne dass die Schlaflabornächte mit in die Auswertung eingehen. Um zu klären, ob die IPT-I auf acht Sitzungen verkürzt werden könnte, waren ebenfalls die Messzeitpunkte BL, S2, S9 betrachtet worden. Die Ergebnisse zeigen, dass sich beide Gruppen in ihren subjektiven quantitativen Schlafparametern veränderten. Für die in beiden Gruppen nachweisbaren Veränderungen von der Baseline bis zum eigentlichen 
Therapiebeginn (S2), die in einem Anstieg der tatsächlichen Schlafzeit (TST) um mehr als 20 Minuten und einer Abnahme der Einschlaflatenz (EL) um ca. zwölf Minuten bestehen, gibt es zwei mögliche Erklärungen: Zum einen können die bereits oben erwähnten Effekte (s. Kapitel 4.4.3, 4.4.1.2.2) vorliegen, andererseits wäre denkbar, dass die Patienten gelernt haben, die quantitativen Parameter genauer einzuschätzen. Der lediglich in der PMR-Gruppe nachgewiesene TST-Anstieg unter der Therapie von weiteren 20 Minuten zeigt, dass diese Therapie als solche eine direkte Wirkung auf die TST hat. Von der IPT-I kann dies dagegen nicht behauptet werden. Einer fehlenden weiteren Senkung der EL unter der PMR-Therapie steht ein direkter Erfolg in der IPT-IGruppe gegenüber, die während der ersten acht Sitzungen ihre EL um mehr als zehn Minuten senken konnte und damit die 30-Minuten-Grenze unterschritt. Das Ausbleiben ähnlicher Effekte in der PMR-Gruppe könnte damit erklärt werden, dass deren EL schon zu Therapiebeginn unter 30 Minuten lag und sie sich daher nur schwer noch weiter senken konnte. Es könnte jedoch auch sein, dass fälschlicherweise die Übungszeit für die PMR in der EL mit enthalten ist (s. Kapitel 4.4.1.2.1).

\subsection{Die Therapeutin}

Sowohl die IPT-I als auch die PMR sind durch dieselbe Therapeutin (Müller-Popkes 1997) durchgeführt worden, die in beiden Therapieformen ausgebildet ist. Sie verfügte über langjährige klinische Erfahrung und über Erfahrung mit ambulanten schlafgestörten Patienten. Sie stand zu Beginn der Durchführung der IPT-I unter ständiger Supervision der Verfasserin des IPT-I-Manuals (Schramm 1993 unveröffentlicht, zit. n. Müller-Popkes 1997).

Die Therapeutin erfüllt damit die von Klerman et al. (1984) aufgestellten Kriterien einer IPT-Therapeutin. Daher können die mäßigen Therapieeffekte nicht auf die Therapeutin zurückgeführt werden.

\subsection{Zusammenfassende Beurteilung und Ausblick}

Zusammenfassend lässt sich sagen, dass beide Therapien positive Auswirkungen auf den Schlaf haben, bei insgesamt mäßigen Effekten ist die PMR-Gruppe der IPT-IGruppe jedoch leicht überlegen. Die große Heterogenität einer kleinen Stichprobe hat möglicherweise dazu geführt, dass Therapieeffekte nicht sichtbar werden. Eine Trennung nach Auswirkung auf ein- und durchschlafgestörte Patienten (Espie 1991) erschien bei dieser kleinen Stichprobe jedoch nicht sinnvoll. Die IPT-I sollte in dieser 
Studie auf ihre Wirksamkeit bei primärer Insomnie überprüft werden. Sie ist sowohl in der Durchführung als auch in der Ausbildung und Supervision des Therapeuten die wesentlich aufwendigere Behandlungsmethode.

Eine für die vorliegende Untersuchung durchgeführte Auswertung zusätzlichen Datenmaterials zeigte als neues Ergebnis eine positive Auswirkung der IPT-I auf die Häufigkeit der Schlafunterbrechungen. In Anbetracht der im Vergleich zur PMR keinesfalls überzeugenderen Daten bezüglich der Schlafverbesserung wäre zu überlegen, die IPT-I aufzugeben. Zuvor sollte jedoch eine Studie mit einem modifizierten Design mit einer größeren Stichprobe durchgeführt werden. Der Grund der bislang gezeigten mäßigen Wirksamkeit der IPT-I mag jedoch darin liegen, dass die Schlafstörungen der Patienten dieser Studie seit langem chronifiziert sind. Die Urform der IPT-I behandelt die Depression, die im Gegensatz zur Insomnie weniger schleichend beginnt. Wichtig ist daher eine Aufklärung der Bevölkerung über Schlafstörungen, damit Patienten sich frühzeitiger in Behandlung begeben als zu einem Zeitpunkt, an dem die Auslöser nur noch schwer oder gar nicht mehr therapeutisch angehbar sind. Gegen diese Theorie sprechen allerdings Hinweise für einen Zusammenhang zwischen der Dauer der Schlafstörung und den Verbesserungen von Schlafqualität und Tagesbefindlichkeit im Laufe der IPT-I-Behandlung (Müller-Popkes 1997). Eine Folgestudie mit einer größeren Stichprobe würde eine individuelle Auswertung ermöglichen. Eine hier vorausgesetzte Beschränkung der Krankheitsdauer böte die Möglichkeit, den Auslöser der Schlafstörung zu therapieren, bevor sich die Insomnie verselbständigt. Einjahreskatamnesen könnten eine verlässliche Auskunft über die Stabilität des Behandlungserfolges geben. Die explorative Datenanalyse hat ergeben, dass die Verkürzung der Therapien zunächst keinen nachteiligen Effekt erwarten lässt, auch wenn über die Aufrechterhaltung der Effekte über das Behandlungsende hinaus keine Aussagen gemacht werden können. Eine Umsetzung in einer Folgestudie mit einer Beschränkung der Behandlungsdauer auf acht Sitzungen wäre denkbar.

Vor folgendem Hintergrund wäre eine Modifikation der IPT-I denkbar: In der Adaption der IPT für Patientinnen mit Bulimie (Fairburn 1993) wurde bewusst die Behandlung der Symptomatik außen vor gelassen und sich ausschließlich auf die Behandlung der interpersonellen Problematik konzentriert, darin unterschied sich diese vor allem langfristige Erfolge verbuchende Therapie von ihrer Urform. Sie hatte über das Behandlungsende hinausreichend zunehmende Wirkung bis zur Einjahreskatamnese. Die IPT-I hingegen beschritt den gegensätzlichen Weg, als sie dem Symptom selbst, der Schlafstörung, mehr zeitlichen Raum in der Behandlung gab. Interessant wäre daher 
eine Modifikation der in der vorliegenden Untersuchung dargestellten IPT-I-Form, in der ebenso wie in der Bulimie-Adaption das Symptom, d.h. in diesem Fall die Schlafstörung, nicht thematisiert wird. Hierfür dürfte jedoch die Akzeptanz der Insomniepatienten fehlen, die die Symptomorientierung vermissen (Müller-Popkes 1997), vor allem wenn ihnen im Falle der Chronifizierung der Zusammenhang zwischen der Schlafstörung und auslösenden Ursachen nicht ersichtlich ist. Die Bulimie ist zwar ebenfalls wie die primäre Insomnie eine zur Chronifizierung neigende Störung; eine ausschließlich ursachenorientierte Therapie der chronifizierten, sich von der auslösenden Ursache verselbständigten Schlafstörung wird jedoch als nicht aussichtsreich betrachtet (Hajak und Rüther 1991b), oft ist diese Ursache nach jahrelanger Insomnie auch nicht mehr erkennbar (Steinberg et al. 1987). Ist die Ursache bekannt, so erscheint grundsätzlich eine individuellere Annäherung an die spezifische zugrunde liegende Problematik erfolgversprechend zu sein: Eine spezifische TrauerBehandlung zeigte bei Shear et al. 2005 mit 51\% eine höhere und schnellere Ansprechrate als die Anwendung der Interpersonellen Psychotherapie mit 28\%, die ebenfalls auf die Trauer den Behandlungsfokus legen kann. Dieser Effekt mag an der höheren Spezialisierung dieser Behandlung auf die Ursache liegen. Der folgende Ansatz zur verbesserten Wirksamkeit der IPT zur Behandlung depressiver Patienten ließe sich möglicherweise auch auf die Insomniepatienten übertragen: Caspar et al. 2005 konnten die selbst aufgestellte Hypothese teilweise bestätigen, dass die Komplementarität des Therapeutenverhalten mit der durch die Patienten (aber nicht durch andere) als erfolgreich beurteilten Veränderung korreliert. Das heißt, dass der Therapeut dem Patienten spontan eine maßgeschneiderte Beziehung anbietet, die sich auf die wichtigsten Ziele des Patienten konzentriert. Die Therapie der chronifizierten Schlafstörung stellt weiterhin eine Herausforderung dar, der mit einem individuelleren therapeutischen Zuschnitt auf die Ursache der Schlafstörung und die Bedürfnisse des Patienten begegnet werden könnte. 


\section{Zusammenfassung}

Ein- und Durchschlafstörungen, die mehr als dreimal pro Woche über mindestens vier Wochen auftreten und zu Einbußen in der Leistungsfähigkeit am Tage führen, sind die Leitsymptome des Krankheitsbildes der überwiegend chronischen Insomnie. Zu den prädisponierenden und auslösenden Faktoren einer Insomnie zählen einschneidende Lebensereignisse, mangelnde soziale Kompetenz sowie die Neigung, vor allem negative Gefühle in sich aufzunehmen. Eine Therapieform, die diese Faktoren gezielt bearbeitet, ist die Interpersonelle Psychotherapie (IPT), die an der Bearbeitung zwischenmenschlicher Probleme ansetzt und für die Behandlung von Depressionen entwickelt wurde. Die IPT wurde für Insomniepatienten modifiziert (IPT-I) und deren Wirkung auf den Schlaf in einer randomisierten und kontrollierten Studie untersucht.

25 Patienten mit einer primären Insomnie (Alter 47 \pm 19 Jahre, 11m 14w, Dauer der Erkrankung: Mittelwert 9 Jahre, Min. 0,5 Max. 35 Jahre) wurden nach Ausschluss einer organischen oder psychiatrischen Ursache der Schlafstörung nach dem Zufallsprinzip in zwei Behandlungsgruppen eingeteilt und entweder mit IPT-I oder Progressiver Muskelrelaxation nach Jacobson (PMR) behandelt. Das jeweilige Verfahren wurde durch dieselbe Therapeutin in zwölf wöchentlichen Einzelsitzungen durchgeführt.

Der Therapieerfolg wurde mittels stationärer polysomnografischer Ableitungen im Schlaflabor jeweils zwei Nächte vor und nach der Behandlung gemessen sowie anhand von Patientenfragebögen evaluiert mit Angaben zu subjektiven qualitativen und quantitativen Schlafparametern vor, während und direkt nach der Behandlung. In diesen Fragebögen beurteilten die Patienten den Schlaf rückblickend über einen zweiwöchigen Zeitraum sowie täglich den Schlaf der vergangenen Nacht.

Die vorliegende Untersuchung betrachtet über die subjektiven und objektiven Schlafveränderungen vor und nach den Behandlungen hinaus die subjektive Beurteilung des Schlafes im Verlauf der Behandlungen und die Möglichkeit einer Therapieverkürzung.

Die im Schlaflabor untersuchten objektiven Parameter zeigten in der IPT-I-Gruppe tendenzielle Besserungen aller vier untersuchten Schlafparameter Schlafeffizienz, Einschlaflatenz, Gesamtschlafzeit und nächtliche Wachzeit, in der PMR-Gruppe nur der beiden letztgenannten Parameter. 
Die Auswirkungen der beiden Therapieformen auf den bereits prätherapeutisch nicht sehr beeinträchtigten subjektiven Schlaf zeigten sich zu den Messzeitpunkten der Schlaflabornächte vor und nach der Behandlung als gering: In der täglichen Schlafbeurteilung verbesserte sich die IPT-I-Gruppe in der Einschlaflatenz sowie tendentiell in den qualitativen Schlafparametern und in der Häufigkeit der Schlafunterbrechungen, in der PMR-Gruppe verschlechterten sich dagegen tendentiell Einschlaflatenz und Schlafqualität. In der Zwei-Wochen-Rückschau verbesserten sich die bereits vor Behandlungsbeginn in der PMR-Gruppe höhere Schlafqualität und das morgendliche Erholungsgefühl signifikant nur in der PMR-Gruppe, in der IPT-I-Gruppe verbesserte sich nur das morgendliche Erholungsgefühl lediglich tendentiell. Die Schlafquantität zeigte sich nur in einzelnen Parametern signifikant gebessert: Die nächtliche Wachzeit nahm in beiden Gruppen und die Einschlaflatenz nahm in der PMR-Gruppe annähernd signifikant ab.

Die Beurteilungen der subjektiven Parameter in den während des gesamten Behandlungszeitraumes täglich ausgefüllten Patientenfragebögen wichen von den Ergebnissen der vorangegangenen Untersuchung über dieselbe Stichprobe (MüllerPopkes 1997) vor allem aus zwei Gründen ab: 1. Prätherapeutische Veränderungen und 2. Abweichendes Schlafverhalten und / oder abweichende Schlafbeurteilung im Schlaflabor. In der täglichen Verlaufsbetrachtung während des gesamten Behandlungszeitraumes zeigte sich in der IPT-I-Gruppe in der mittleren Phase eine tendenzielle Verschlechterung von Gesamtschlafzeit, Schlafqualität und morgendlichem Erholungsgefühl. Ursache könnte vermehrte psychische Belastung durch die Problembearbeitung während des mittleren Behandlungsabschnitts sein.

Bereits nach acht Sitzungen wurden in beiden Gruppen signifikante Verbesserungen der subjektiven Schlafquantität deutlich: Die IPT-I-Gruppe schlief schneller ein, die PMRGruppe schlief länger. Die PMR-Gruppe konnte zudem tendenziell ihre subjektive Schlafqualität verbessern.

Beide Therapien zeigten in dieser Untersuchung insgesamt mäßige Effekte mit größerer Wirksamkeit der PMR vor allem auf den subjektiven Schlaf. Die IPT-I blieb dagegen angesichts des im Vergleich größeren Aufwandes hinter den Erwartungen zurück. Aufgrund der kleinen heterogenen Stichprobe wurden Therapieeffekte vermutlich nicht abgebildet. Ein längerer Nachbeobachtungszeitraum zur Beurteilung der Langzeitwirkung der IPT-I auf die chronifizierte Schlafstörung wäre zur Beurteilung der klinischen Relevanz wünschenswert. 


\section{Anhang}

ถุ

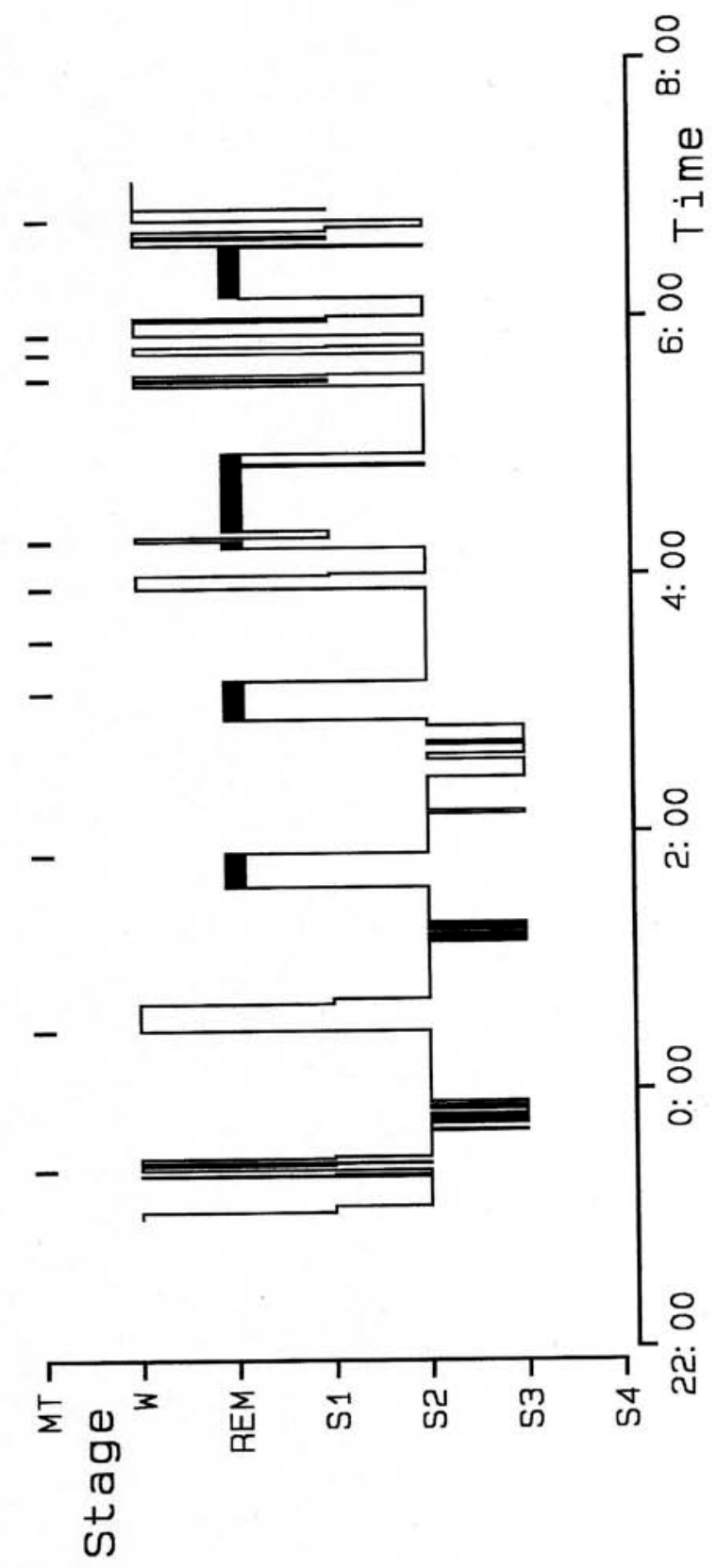




\begin{tabular}{llr} 
GESAMTE ZEIT & IM BETT \\
\hline \hline Beginn & $=$ & $23: 00: 00$ \\
Ende & $=$ & $7: 03: 30$ \\
Dauer & $=$ & $8: 03: 30$
\end{tabular}

\begin{tabular}{|c|c|c|c|c|}
\hline & EPOCHEN & DAUER & LATENZ & \%TIB \\
\hline $\begin{array}{l}\text { TIB } \\
\text { SPT } \\
\text { TST } \\
\text { SWS }\end{array}$ & $\begin{array}{r}967 \\
929 \\
830 \\
67\end{array}$ & $\begin{array}{l}8: 03: 30 \\
7: 44: 30 \\
6: 55: 00 \\
0: 33: 30\end{array}$ & & $\begin{array}{r}96.1 \\
85.8 \\
6.9\end{array}$ \\
\hline $\begin{array}{r}\text { FZ } \\
\text { MT } \\
\text { W } \\
\text { REM } \\
\text { S1 } \\
\text { S2 } \\
\text { S3 } \\
\text { S4 }\end{array}$ & $\begin{array}{r}0 \\
11 \\
131 \\
190 \\
38 \\
530 \\
67 \\
0\end{array}$ & $\begin{array}{l}0: 05: 30 \\
1: 05: 30 \\
1: 35: 00 \\
0: 19: 00 \\
4: 25: 00 \\
0: 33: 30\end{array}$ & $\begin{array}{l}0: 22: 00 \\
2: 34: 30 \\
0: 03: 00 \\
0: 06: 00 \\
0: 41: 00\end{array}$ & $\begin{array}{r}1.1 \\
13.5 \\
19.6 \\
3.9 \\
54.8 \\
6.9\end{array}$ \\
\hline
\end{tabular}

GESAMTE SCHLAFPERIODE

\begin{tabular}{llr}
\hline \hline Latenz & $=$ & $0: 06: 00$ \\
Beginn & $=$ & $23: 06: 00$ \\
Ende & $=$ & $6: 50: 30$ \\
Dauer & $=$ & $7: 44: 30$
\end{tabular}

\begin{tabular}{|c|c|c|c|c|}
\hline & EPOCHEN & DAUER & $\%$ SPT & \%TST \\
\hline $\begin{array}{l}\text { SPT } \\
\text { TST } \\
\text { SWS }\end{array}$ & $\begin{array}{r}929 \\
830 \\
67\end{array}$ & $\begin{array}{l}7: 44: 30 \\
6: 55: 00 \\
0: 33: 30\end{array}$ & $\begin{array}{r}89.3 \\
7.2\end{array}$ & 8.1 \\
\hline $\begin{array}{r}\text { FZ } \\
\text { MT } \\
\text { W } \\
\text { REM } \\
\text { S1 } \\
\text { S2 } \\
\text { S3 } \\
\text { S4 }\end{array}$ & $\begin{array}{r}0 \\
11 \\
99 \\
190 \\
32 \\
530 \\
67 \\
0\end{array}$ & $\begin{array}{l}0: 05: 30 \\
0: 49: 30 \\
1: 35: 00 \\
0: 16: 00 \\
4: 25: 00 \\
0: 33: 30\end{array}$ & $\begin{array}{r}1.2 \\
10.7 \\
20.5 \\
3.4 \\
57.1 \\
7.2\end{array}$ & $\begin{array}{r}1.3 \\
22.9 \\
3.9 \\
63.9 \\
8.1\end{array}$ \\
\hline
\end{tabular}




\section{Literaturverzeichnis}

Agnew HW, Webb WB and Williams RL (1966): The first night effect: an EEG study of sleep. Psychophysiology 2, 263-266

Ancoli-Israel S, Cole R, Alessi C, Chambers M, Moorcroft W, Pollak CP (2003): The role of actigraphy in the study of sleep and circadian rythms. Sleep $\underline{26}$ (3), 342-392

Anderer P, Gruber G, Parapatics S, Woertz M, Miazhynskaia T, Klosch G, Saletu B, Zeitlhofer J, Barbanoj MJ, Danker-Hopfe H, Himanen SL, Kemp B, Penzel T, Grozinger M, Kunz D, Rappelsberger P, Schlogl A, Dorffner G (2005): An E-health solution for automatic sleep classification according to Rechtschaffen and Kales: validation study of the Somnolyzer $24 \times 7$ utilizing the Siesta database. Neuropsychobiology $\underline{51}$ (3), 115-133

APA 1987 = American Psychiatric Association: Diagnostisches und statistisches Manual psychischer Störungen (DSM-III-R). Deutsche Bearbeitung und Einführung von Wittchen H-U, Saß H, Zaudig M, Köhler K. Beltz, Weinheim 1987

APA $1996=$ American Psychiatric Association: Diagnostisches und statistisches Manual psychischer Störungen (DSM-IV). Deutsche Bearbeitung und Einführung von Saß H, Wittchen H-U, Zaudig M. Hogrefe-Verlag, Göttingen 1996

ASDA = American Sleep Disorders Association (1992): EEG arousals: scoring rules and examples: a preliminary report from the Sleep Disorders Atlas Task Force of the American Sleep Disorders Association. Sleep 15 (2), 173-184

ASDA $=$ American Sleep Disorders Association: The international classification of sleep disorders. Rochester MN 2001

ASDC = Association of Sleep Disorders Centers (1979): Diagnostic classification of sleep and arousal disorders. Sleep 2, 1-137

Beck JS: Praxis der kognitiven Therapie. Beltz, Weinheim 1995

Bernstein DA, Borkovec TD: Progressive relaxation technique: a manual for the helping professions. Research Press Company, Champaign IL 1973 
Bernstein DA, Borkovec TD: Entspannungstraining. Handbuch der progressiven Muskelentspannung. 7. Auflage, (Reihe „Leben lernen“ Nr.16), Verlag J. Pfeiffer, München 1995

Bixler EO, Kales A, Leo LE, Slye T (1973): A comparison of subjective estimates and objective sleep laboratory findings in insomniac patients. Sleep Res 2, 134

Bonnet MH, Arand DL (1997): Physiological activation in patients with Sleep State Misperception. Psychosom Med $\underline{59}$ (5), 533-540

Bonnet MH, Arand DL (1998): Heart rate variability in insomniacs and matched normal sleepers. Psychosom Med $\underline{60}$ (5), 610-615

Bootzin RR: A comparison of stimulus control instructions and progressive relaxation training in the treatment of sleep-onset insomnia. Northwestern University, Evanston IL 1975, unveröffentlicht; zit. n. Espie 1991, 178-180

Bootzin RR and Nicassio PM: Behavioral treatments for insomnia; in: Progress in behavior modification, Vol. 6; hrsg. v. Hersen M, Eisler RM, Miller PM; Academic Press, New York 1978, 1-45

Borkovec TD (1982): Insomnia. J Consult Clin Psychol 무, 880-895

Borkovec TD, Fowles D (1973): Controlled investigation of the effects of progressive relaxation and hypnotic relaxation on insomnia. J Abnorm Psychol 2, 153158Borkovec TD, Weerts TC (1976): Effects of progressive relaxation on sleep disturbance: An electroencephalic evaluation. Psychosom Med $\underline{38}$, 173-180

Borkovec TD, Hennings BL (1978): The role of physiological attention-focusing in the relaxation treatment of sleep disturbance, general tension and specific stress reaction. Behav Res Ther $\underline{16}$, 7-20

Borkovec TD, Steinmark SW, Nau SD (1973): Relaxation training and single item desensitization in the group treatment of insomnia. J Behav Ther Exp Psychiatry $\underline{4}$, 401410

Borkovec TD, Kaloupek DG, Slama KM (1975): The facilitative effect of muscle tension-release in the relaxation treatment of sleep disturbance. Behav Ther $\underline{6}, 301-309$ 
Borkovec TD, Grayson JB, O`Brien GT, Weerts TC (1979): Relaxation treatment of pseudoinsomnia and idiopathic insomnia: An electroencephalographic evaluation. J Appl Behav Anal 12, 37-54

Bortz J: Lehrbuch der Statistik. Für Sozialwissenschaftler. Korrigierter Nachdruck der ersten Auflage, Springer, Berlin 1979

Bortz J, Lienert GA, Boehnke K: Verteilungsfreie Methoden in der Biostatistik. Springer, Berlin 1990

Bowlby J: Attachment. Basic Books, New York 1969

Brosius G und Brosius F: SPSS Base System und Professional Statistics. International Thomson Publishing, Bonn 1995

Brown GW, Harris T: Social origins of depression: a study of psychiatric disorders in women. Tavistock, London 1978

Brown GW, Harris TO, Copeland JR: Depression und Verlustereignisse; in: Sozialer Streß und psychische Erkrankung. Lebensverändernde Ereignisse als Ursache seelischer Störungen; hrsg. v. Katschnig H; Urban \& Schwarzenberg, München 1980, 308-333

Brown GW, Andrews B, Harris T (1986): Social support, self-esteem and depression, Psychol Med $\underline{16}$, 813-831

Brummett BH, Krystal AD, Siegler IC, Kuhn C, Surwit RS, Züchner S, Ashley-Koch A, Barefoot JC, Williams RB (2007): Associations of a regulatory polymorphism of monoamine oxidase-A gene promoter (MAOA-uVNTR) with symptoms of depression and sleep quality. Psychosom Med $\underline{69}$ (5), 396-401

Brusco LI, Fainstein I, Marquez M, Cardinali DP (1999): Effect of melatonin in selected populations of sleep-disturbed patients. Biol Signals Recept $\underline{8}$ (1-2), 126-131

Bühl A, Zöfel P: SPSS für Windows Version 6.1. Praxisorientierte Einführung in die moderne Datenanalyse. 3.Auflage; Addison-Wesley-Longman, Bonn 1996

Buysse DJ, Reynolds CF III, Monk TH, Bermon SR and Kupfer DJ (1989): The Pittsburgh Sleep Quality Index: a new instrument for psychiatric practice and research. Psychiatry Res 28, 193-213; (deutsche Übersetzung: Schramm E: Pittsburgher Schlafqualitätsindex. Freiburg; in: Müller-Popkes K: Interpersonelle Psychotherapie zur 
Behandlung von Patienten mit primärer Insomnie: Ein Vergleich mit progressiver Muskelrelaxation. Cuvillier-Verlag, Göttingen 1997; Anhang)

Carskadon MA, Dement WC, Mitler MM, Guilleminault C, Zarcone VP, Spiegel R (1976): Self-reports versus sleep laboratory findings in 122 drug-free subjects with complaints of chronic insomnia. Am J Psychiatry 133, 1382-1388

Carroll KM, Rounsaville BJ, Gawin FH (1991): A comparative trial of psychotherapies for ambulatory cocaine abusers: relapse prevention and interpersonal psychotherapy. Am J Drug Alcohol Abuse 17 (3), 229-247

Caspar F, Grossmann C, Unmüssig C, Schramm E (2005): Complementary therapeutic relationship: Therapist behavior, interpersonal patterns, and therapeutic effects. Psychother Res $\underline{15}$ (1-2), 91-102

Cautela JR, Wisocki PA (1977): The thought stopping procedure: Description, application and learning theory interpretations. Psychol Rep 2, 255-264

Cernovsky ZZ (1984): Lifestress measures and reported frequency of sleep disorders. Percept Mot Skills $\underline{58}$, 39-49

Chambers MJ (1992): Therapeutic issues in the behavioral treatment of insomnia. Prof Psychol: Res Pract 23, 131-138

Chediak A, Esparis B, Isaacson R, de la Cruz L, Ramirez J, Rodriguez JF, Abreu A (2006): How many polysomnograms must sleep fellows score before becoming proficient at scoring sleep? J Clin Sleep Med 2 (4), 427-430

Cheek RE, Shaver JL, Lentz MJ (2004): Variations in sleep hygiene practices of women with and without insomnia. Res Nurs Health 27 (4), 225-236

Clarenbach P, Steinberg R, Weess HG, Berger M (1995): Empfehlung zu Diagnostik und Therapie der Insomnie. Deutsche Gesellschaft für Schlafforschung und Schlafmedizin DGSM. Nervenarzt $\underline{66}$ (10), 723-729

Coates TJ , Killen JD, George J, Marchini E, Silverman S, Hamilton S, Thorensen CE (1982): Discriminating good sleepers from insomniacs using all-night polysomnograms conducted at home. J Nerv Ment Dis $\underline{170}$, 224-230 
Coates TJ, Killen JD, Silverman S, George J, Marchini E, Hamilton S, Thoresen CE (1983): Cognitive activity, sleep disturbance, and stage specific differences between reported and recorded sleep. Psychopharmacology (Berl) 20, 243-250

Collop NA (2002): Scoring variability between polysomnography technologists in different sleep laboratories. Sleep Med $\underline{3}$ (1), 43-47

Coursey RD, Buchsbaum M, Frankel BL (1975): Personality measures and evoked responses in chronic insomniacs. J Abnorm Psychol 4ㅗ, 239-249

Coyle K, Watts FN (1991): The factorial structure of sleep dissatisfaction. Behav Res Ther $\underline{29}$ (6), 513-520

Coyne JC (1976): Depression and the response of others. J Abnorm Psychol 모, 186193

Crowley K, Trinder J, Kim Y, Carrington M, Colrain IM (2002): The effects of normal aging on sleep spindle and K-complex production. Clin Neurophysiol $\underline{113}$ (19), 16151622

Dement W, Seidel W, Carskadon C (1984): Issues in the diagnosis and treatment of insomnia. Psychopharmacology (Berl) Supplementum $\underline{1}, 12-43$

Dijk DJ, Duffy JF, Czeisler CA (2001): Age-related increase in awakenings: impaired consolidation of nonREM sleep at all circadian phases. Sleep 24 (5), 565-577

DiMascio A, Weissman MM, Prusoff BA, Neu C, Zwilling M, Klerman GL (1979): Differential symptom reduction by drugs and psychotherapy in acute depression. Arch Gen Psychiatry $\underline{36}$, 1450-1456

Eberlein G: Gesund durch Autogenes Training. ECON Taschenbuch Verlag Düsseldorf 1996

Edinger JD, Stout AL, Hoelscher TJ (1988): Cluster analysis of insomniacs’ MMPI profiles: relation of subtypes to sleep history and treatment outcome. Psychosom Med 50, 77-87

Edinger JD, Wohlgemuth WK, Radtke RA, Marsh GR, Quillian RE (2001): Does cognitive-behavioral insomnia therapy alter dysfunctional beliefs about sleep? Sleep $\underline{24}$ (5), 591-599 
Ehlers CL, Frank E, Kupfer DJ (1988): Social zeitgebers and biological rhythms: a unified approach to understanding the etiology of depression. Arch Gen Psychiatry $\underline{45}$, 948-952

Elkin I, Shea MT, Watkins JT, Imber SD, Sotsky SM, Collins JF, Glass DR, Pilkonis PA, Leber WR, Docherty JP et al. (1989): National Institute of Mental Health Treatment of Depression Collaborative Research Program: general effectiveness of treatment. Arch Gen Psychiatry 46, 971-983

Engel RR, Knab B: Theoretische Vorstellungen zur Genese von Schlafstörungen; in: Psychophysiologische Merkmale klinischer Symptome; 1. Psychophysiologische Dysfunktionen; hrsg. v. Vaitl D, Knapp TW, Birbaumer N, Dt. Gesellschaft für Psychologie; Beltz, Weinheim 1985, 128-142

Erman MK, Loewy DB, Scharf MB (2005): Effects of temazepam $7.5 \mathrm{mg}$ and temazepam $15 \mathrm{mg}$ on sleep maintenance and sleep architecture in a model of transient insomnia. Curr Med Res Opin 21 (2), 223-30

Espie CA: The psychological treatment of insomnia. John Wiley \& Sons, Chichester 1991

Espie CA, Lindsay WR (1987): Cognitive strategies for the management of severe sleep-maintenance insomnia: A preliminary investigation. Behav Psychother $\underline{15}$, 338395

Espie CA, Lindsay WR, Brooks DN, Hood DM, Turvey T (1989): A controlled comparative investigation of psychological treatments for chronic sleep-onset insomnia. Behav Res Ther 르, 79-88

Espie CA, MacMahon KM, Kelly HL, Broomfield NM, Douglas NJ, Engleman HM, McKinstry B, Morin CM, Walker A, Wilson P (2007): Randomized clinical effectiveness trial of nurse-administered small-group cognitive behavior therapy for persistent insomnia in general practice. Sleep $\underline{30}$ (5), 574-584

Fahrenberg J, Hampel R, Selg H: Das Freiburger Persönlichkeitsinventar FPI. Revidierte Fassung FPI-R und teilweise geänderte Fassung FPI-A1. Handanweisung. 4. Auflage, Hogrefe-Verlag, Göttingen 1984

Fairburn CG: Interpersonal psychotherapy for bulimia nervosa; in: New applications of interpersonal psychotherapy; hrsg. v. Klerman GL, Weissman MM; American Psychiatric Press, Washington DC 1993, 353-378 
Fairburn CG, Jones R, Peveler RC, Carr SJ, Solomon RA, O`Connor ME, Burton J, Hope RA (1991): Three psychological treatments for bulimia nervosa: a comparative trial. Arch Gen Psychiatry 48, 463-469

Fairburn CG, Jones R, Peveler RC, Hope RA, O`Connor M (1993): Psychotherapy and bulimia nervosa: longer-term effects of interpersonal psychotherapy, behavior therapy and cognitive therapy. Arch Gen Psychiatry 50, 419-428

Floyd JA, Medler SM, Ager JW, Janisse JJ (1989): Age-related changes in initiation and maintenance of sleep: a meta-analysis. Res Nurs Health $\underline{23}$ (2), 106-117

Foley SH, Rounsaville BJ, Weissman MM, Sholomskas D, Chevron E (1989): Individual versus conjoint interpersonal psychotherapy for depressed patients with marital disputes. Int J Fam Psychiatry $\underline{10}$, 29-42

Ford DE, Kamerow DB (1989): Epidemiologic study of sleep disturbances and psychiatric disorders. JAMA 262, 1479-1484

Frank E (1991): Interpersonal psychotherapy as a maintenance therapy for patients with recurrent depression. Psychotherapy 28, 259-266

Frank E, Frankel D, Carter S, Cornes C, Kupfer DJ: Manual of the adaption of interpersonal psychotherapy to the treatment of bipolar disorder. Western Psychiatric Institute and Clinic, Pittsburgh PA 1990a, unveröffentlicht; zit. n. Müller-Popkes 1997, 71-72

Frank E, Kupfer DJ, Perel JM (1990b): Three year outcomes for maintenance therapies in recurrent depression. Arch Gen Psychiatry 47, 1093-1099

Frank E, Kupfer DJ Walter EF, McEachran AB, Cornes C (1991): Efficacy of interpersonal psychotherapy as a maintenance treatment for recurrent depression: contributing factors. Arch Gen Psychiatry $\underline{48}$, 1053-1059

Frank E, Frank N, Cornes C, Imber SD, Miller MD, Morris SM, Reynolds CF III.: Interpersonal psychotherapy in the treatment of late-life depression; in: New applications of interpersonal psychotherapy; hrsg. v. Klerman GL, Weissman MM; American Psychiatric Press, Washington DC 1993, 167-198

Frankel BL, Coursey RD, Buchbinder R, Snyder F (1976): Recorded and reported sleep in chronic primary insomnia. Arch Gen Psychiatry $\underline{33}$, 615-623 
Freedman R, Papsdorf J (1976): Biofeedback and progressive relaxation treatment of sleep-onset insomnia: a controlled all-night investigation. Biofeedback Self Regul $\underline{1}$, 253-271

Friebel V, Friedrich S (1992): Entspannungsverfahren bei Schlafstörungen. Krankenhauspsychiatrie $\underline{3}$, 82-87

Friedman L, Bliwise DL, Yesavage JL, Salom SR (1991): A preliminary study comparing sleep restriction and relaxation treatments for insomnia in older adults. $\mathrm{J}$ Gerontol: Psychol Sci $\underline{46}$ (1), 1-8

Garfield SL (1981): Evaluating the psychotherapies. Behav Ther 12, 295-307

Gershman L, Clouser RA (1974): Treating insomnia with relaxation and desensitization in a group setting by an automated approach. J Behav Ther Exp Psychiatry $\underline{5}$, 31-35

Gillin JC (1998): Are sleep disturbances risk factors for anxiety, depressive and addictive disorders? Acta Psychiatr Scand 98 (Suppl 393), 39-43

Gillin JC, Byerley WF (1990): The diagnosis and management of insomnia. N Engl J Med 322, 239-248

Görtelmeyer R: Schlaffragebogen A und B (SF-A und SF-B). Selbstbeurteilungsskala (S); in: Internationale Skalen für Psychiatrie; hrsg. v. Collegium Internationale Psychiatriae Scalarum (CIPS); Beltz, Weinheim 1981, o.Sz.

Haba-Rubio J, Ibanez V, Sforza E (2004): An alternative measure of sleep fragmentation in clinical practice: the sleep fragmentation index. Sleep med $\underline{5}(6), 577-$ 581

Hager W, Westermann R (1983): Entscheidung über statistische und wissenschaftliche Hypothesen: Probleme bei mehrfachen Signifikanztests zur Prüfung einer wissenschaftlichen Hypothese. Z Sozialpsychol 14, 106-117

Hajak G, Rüther E: Chronic insomnia in the elderly; in: Biological Psychiatry, vol. I; hrsg.v. Racagni G, Brunello N, Fukendo T; Elsevier, Amsterdam 1991a, 845-848

Hajak G, Rüther E: Chronische Insomnien - ein diagnostisches und therapeutisches Problemfeld; in: Schlaf; hrsg. v. Steinberg R; Tilia, Klingenmünster 1991b, 60-64 
Hajak G, Rüther E: Therapie von Ein- und Durchschlafstörungen; in: Handbuch der Therapie psychiatrischer Erkrankungen; hrsg. v. Möller HJ; Enke, Stuttgart 1993, 663704

Hajak G, Rüther E: Insomnie. Springer, Berlin 1995

Hajak G, Müller-Popkes K, Riemann D, Mayer G, Lauer C, Backhaus J, Crönlein T, Hoffmann M, Kästner E, Löchte B et al. (1997): Psychologische, psychotherapeutische und andere nichtpharmakologische Therapieformen zur Behandlung der Insomnie. Fortschr Neurol Psychiat $\underline{65}$, 133-144

Hajak G, Rodenbeck A, Vorderholzer U, Riemann D, Cohrs S, Hohagen F, Berger M, Rüther E (2001): Doxepin in the treatment of primary insomnia: a placebo-controlled, double-blind, polysomnographic study. J Clin Psychiatry $\underline{62}$ (6), 453-463

Halász P, Terzano M, Parrino L, Bódizs R (2004): The nature of arousal in sleep. J Sleep Res $\underline{13}$ (1), 1-23

Hall M, Vasko R, Buysse D, Ombao H, Chen Q, Cashmere JD, Kupfer D, Thayer JF (2004): Acute stress affects heart rate variability during sleep. Psychosom Med $\underline{66}$ (1), 56-62

Hall M, Thayer JF, Germain A, Moul D, Vasco R, Puhl M, Miewald J, Buysse DJ (2007): Psychological stress is associated with heightened physiological arousal during NREM sleep in primary insomnia. Behav Sleep Med $\underline{5}, 178-193$

Hammer N, Todorova A, Hofmann HC, Schober F, Vonderheid-Guth B, Dimpfel W (2001): Description of healthy and disturbed sleep by means of the spectral frequency index (SFx) - a retrospective analysis. Eur J Med Res $\underline{6}$ (8), 333-344

Hatoum HT, Kong SX, Kania CM, Wong JM, Mendelson WB (1998): Insomnia, healthrelated quality of life and healthcare resource consumption. A study of managed-care organisation enrollees. Pharmacoeconomics $\underline{14}$ (6), 629-637

Harris J, Lack L, Wright H, Gradisar M, Brooks A (2007): Intensive Sleep Retraining treatment for chronic primary insomnia: a preliminary investigation. J Sleep Res $\underline{16}$ (3), 276-284

Hauri P: What can insomniacs teach us about the functions of sleep? In: The functions of sleep, hrsg. v. Drucker-Colin R, Shkurovick M, Sterman MB; Academic Press, New York 1979, 251-271 
Hauri P (1981): Treating psychophysiologic insomnia with biofeedback. Arch Gen Psychiatry $\underline{38}, 752$

Hauri P: Primary insomnia; in: Principles and practice of sleep medicine; hrsg. v. Kryger MH, Roth T, Dement WC; Saunders, Philadelphia PA 1989, 442-447

Hauri PJ (1997): Can we mix behavioral therapy with hypnotics when treating insomniacs? Sleep 20 (12), 1111-1118

Hauri PJ, Esther MS (1990): Insomnia. Mayo Clin Proc 65, 869-882

Haynes SN, Woodward S, Moran R, Alexander D (1974): Relaxation treatment of insomnia. Behav Ther $\underline{5}$, 555-558

Healey ES, Kales A, Lawrence JM, Bixler EO, Chamberlin K, Soldatos CR (1981): Onset of insomnia. Role of life-stress events. Psychosom Med 43 (5), 439-451

Heath AC, Kendler KS, Eaves LJ, Martin NG (1990): Evidence for genetic influences on sleep disturbance and sleep pattern in twins. Sleep $\underline{13}$ (4), 318-335

Henderson S (1977): The social network, support and neurosis: the function of attachment in social life. Br J Psychiatry $\underline{131}$, 185-191

Herrera-Arellano A, Luna-Villegas G, Cuevas-Uriostegui ML, Alvarez L, VargasPineda G, Zamilpa-Alvarez A, Tortoriello J (2001): Polysomnographic evaluation of the hypnotic effect of Valeriana edulis standardized extract in patients suffering from insomnia. Planta medica $\underline{67}$ (8), 695-699

Herrmann-Maurer EK, Schneider-Helmert D, Schoenenberger GA (1988): One personality pattern for all insomniacs is a myth. Sleep Res $\underline{17}, 125$

Herzog DB, Keller MB, Lavori PW, Sacks NR (1991): The course and outcome of bulimia nervosa. J Clin Psychiatry $\underline{52}$ (10, suppl), 4-8

Hoffmann SO (1980): Psychodynamik und Therapie von Schlafstörungen. Internist Prax 20, 495-500

Hohagen F, Rinke K, Schramm E, Riemann D, Weyerer S, Berger M (1993): Prevalence and treatment of insomnia in general practice. A longitudinal study. Eur Arch Psychiatry Clin Neurosci 242, 329-336

Hohagen F, Käppler C, Schramm E, Riemann D, Weyerer S, Berger M (1994): Sleep onset insomnia, sleep maintaining insomnia and insomnia with early morning 
awakening - temporal stability of subtypes in a longitudinal study on general practice attenders. Sleep 17, 551-554

Holzrichter S, Hajak G, Rudolf G, Schlaf G, Westenhöfer J, Rodenbeck A, Rabba J, Pudel V, Rüther E (1994): Schlafbeschwerden in West-Deutschland - Eine Repräsentativumfrage. Wien Med Wochenschr, Sonderheft, 62-73

Horowitz M: Stress response syndromes. Jason Aronson, New York 1976

Horowitz LM, Strauß B, Kordy H: Inventar zur Erfassung interpersonaler Probleme. Deutsche Version (IIP-D). Beltz, Weinheim 1994

Huang YL, Liu RY, Wang QS, Van Someren EJ, Xu H, Zhou JN (2002): Ageassociated difference in circadian sleep-wake and rest-activity rythms. Physiol Behav $\underline{76}$ (4-5), 597-603

Huupponen E, Gómez-Herrero G, Saastamoinen A, Värri A, Hasan J, Himanen SL (2007): development and comparison of four sleep spindle detection methods. Artif Intell Med $\underline{40}$ (3), 157-70

Iwata K, Makao M, Yamamoto M, Kimura M (2000): Differentiation of physiological states under sensory deprivation. Method Inf Med $\underline{39}$ (2), 168-170

Jacobs GD, Benson H, Friedman R (1996): Perceived benefits in a behavioral-medicine insomnia program: a clinical report. Am J Med 100, 212-216

Jacobson E: Progressive relaxation. University of Chicago Press, Chicago 1938

Kales A, Kales JD: Evaluation and treatment of insomnia. Oxford University Press, New York 1984

Kales A, Caldwell AB, Preston TA, Healey S, Kales JD (1976): Personality patterns in insomnia. Theoretical implications. Arch Gen Psychiatry 33, 1128-1134

Kales A, Caldwell AB, Soldatos CR, Bixler EO, Kales JD (1983): Biopsychobehavioural correlates of insomnia, II: Pattern specifity and consistency with the MMPI. Psychosom Med $\underline{45}$ (4), 341-356

Kales A, Bixler EO, Vela-Bueno A, Cadieux RJ, Soldatos CF, Kales JD (1984): Biopsychobehavioural correlates of insomnia, III: Polygraphic findings of sleep difficulty and their relationship to psychopathology. Int J Neurosci $\underline{23}$, 43-56 
Kecklund G, Akerstedt T (2004): Apprehension of the subsequent working day is associated with a low amount of slow wave sleep. Biol Psychol $\underline{66}$ (2), 169-176

Kelly MB, Delahaye C, le Gal M (1993): Quality of life and insomnia. Results of the validation studies for a specifically designed questionnaire. JAMA SEA (suppl.) Jan. 1993, 21-26 (incl. appendix: The QOLI questionnaire, 33-36)

Khalsa SB (2005): Treatment of chronic insomnia with yoga: a preliminary study with sleep-wake diaries. Appl Psychophysiol biofeedback 29 (4), 2269-78

Klerman GL, Weissman MM, Rounsaville BJ, Chevron ES: Interpersonal psychotherapy of depression. Basic Books, New York 1984

Kordy H, von Rad M, Senf W (1989): Empirical hypotheses on the psychotherapeutic treatment of psychosomatic patients in short and long-term time-unlimited psychotherapy. Psychother Psychosom $\underline{52}$ (1-3), 155-163

Kunz D, Mahlberg R, Müller C, Tilmann A, Bes F (2004): Melatonin in patients with reduced REM sleep duration: two randomized controlled trials. J Clin Endocrinol Metab $\underline{89}$ (1), 128-134

Lacks P, Rotert M (1986): Knowledge and practice of sleep hygiene techniques in insomniacs and good sleepers. Behav Res Ther 24 (3), 365-368

Lacks P, Powlishta, K (1989): Improvement following behavioral treatment for insomnia: Clinical signifikance, long-term maintenance and predictors of outcome. Behav Ther 20, 117-134

Lacks P, Morin CM (1992): Recent advances in the assessment and treatment of insomnia. J Consult Clin Psychol $\underline{60}$ (4), 586-594

Lacks P, Bertelson AD, Gans L, Kunkel J (1983a): The effectiveness of three behavioral treatments for different degrees of sleep onset insomnia. Behav Ther 14, 593-605

Lacks P, Bertelson AD, Sugarman J, Kunkel J (1983b): The treatment of sleep maintenance insomnia with stimulus-control techniques. Behav Res Ther 21, 291-295

Lavie P (1983): Incidence of sleep apnea in a presumably healthy working population: a significant relationship with excessive daytime sleepiness. Sleep $\underline{6}$, 312-318 
Lamarche CH, Ogilvie RD (1997): Electrophysiological changes during the sleep onset period of psychophysiological insomniacs, psychiatric insomniacs, and normal sleepers. Sleep $\underline{20}$ (9), 724-733

Lawrence PS, Tokarz T: A comparison of relaxation training and stimulus control. Paper presented at the Association for the Advancement of Behavior Therapy, New York 1976, zit. nach Espie 1991, 179-180

Levin YI (1998): "Brain music" in the treatment of patients with insomnia. Neurosci Behav Physiol 28 (3), 330-335

Lichstein KL, Means MK, Noe SL, Aguillard RN (1997): Fatigue and sleep disorders. Behav Res Ther $\underline{35}$ (8), 733-740

Lichstein KL, Riedel BW, Wilson NM, Lester KW, Aguillard RN (2001): Relaxation and sleep compression for late-life insomnia: a placebo-controlled trial. J Consult Clin Psychol $\underline{69}$ (2), 227-239

Lick J, Heffler D (1977): Relaxation training and attention placebo in the treatment of severe insomnia. J Consult Clin Psychol $\underline{45}$, 153-161

Manjunath NK, Telles S (2005): Influence of Yoga and Ayurveda on self-rated sleep in a geriatric population. Indian J Med Res $\underline{121}$ (5), 683-690

McCurry SM, Logsdon RG, Teri L, Vitiello MV (2007): Evidence-based psychological treatments for insomnia in older adults. Psychol Aging 22 (1), 18-27

Marchini EJ, Coates TJ, Magistrad JG, Waldum SJ (1983): What do insomniacs do, think, and feel during the day? A preliminary study. Sleep $\underline{6}, 147-152$

Markowitz JC, Klerman GL, Perry SW, Clougherty KF, Josephs LS: Interpersonal psychotherapy for depressed HIV-seropositive patients; in: New applications of interpersonal psychotherapy; hrsg. v. Klerman GL, Weissman MM; American Psychiatric Press, Washington DC 1993, 199-224

Mellinger GD, Balter MB, Uhlenhuth EH (1985): Insomnia and its treatment. Prevalence and correlates. Arch Gen Psychiatry 42, 225-232

Mendelson WB: Human sleep: research and clinical care. Plenum Press, New York 1987 
Mendelson WB (1993): Insomnia and related sleep disorders. Psychiatr Clin North Am $\underline{16}$ (4), 841-851

Mendelson WB, James SP, Garnett D, Sack DA, Rosenthal NE (1986): A psychophysiological study of insomnia. Psychiatry Res $\underline{19}$, 267-284

Meyer A: Psychobiology: a science of man. CC Thomas, Springfield IL 1957

Montakab H (1999): Akupunktur und Insomnie. Forsch Komplementärmed $\underline{6}$ Suppl. 1, 29-31

Morgenthaler T, Kramer M, Alessi C, Friedman L, Boehlecke B, Brown T, Coleman J, Kapur V, Lee-Chiong T, Owens J, Pancer J, Swick T, American Academy of Sleep Medicine (2006): Practice parameters for the psychological and behavioral treatment of insomnia: an update. An american academy of sleep medicine report. Sleep 29 (11), 1415-1419

Monti JM, Attali P, Monti D, Zipfel A, de la Giclais B, Morselli PL (1994): Zolpidem and rebound insomnia - a double-blind, controlled polysomnographic study in chronic insomniac patients. Pharmacopsychiatry $\underline{27}$ (4), 166-175

Morin CM, Kwentus JA (1988): Behavioral and pharmacological treatments for insomnia. Ann Behav Med 10, 91-100

Morin CM, Gaulier B, Barry T, Kowatch RA (1992): Patients' acceptance of psychological and pharmacological therapies for insomnia. Sleep $\underline{15}$, 302-305

Morin CM, Kowatch RA, Barry T, Walton E (1993): Cognitive-behavior therapy for late-life insomnia. J Consult Clin Psychol $\underline{61}$ (1), 137-146

Morin CM, Culbert JP, Schwartz SM (1994): Nonpharmacological interventions for insomnia: a meta-analysis of treatment efficacy. Am J Psychiatry 151 (8), 1172-1180

Morin CM, Bootzin RR, Buysse DJ, Edinger JD, Espie CA, LichsteinKL (2006): Psychological and behavioral treatment of insomnia: update of the recent evidence (1998-2004). Sleep 29 (11), 1398-1414

Morin AK, Jarvis CI, Lynch AM (2007): Therapeutic options for sleep-maintenance and sleep-onset insomnia. Pharmacotherapy 27 (1), 89-110 
Moul DE, Germain A, Cashmere JD, Quigley M, Miewald JM, Buysse DJ (2007): Examination initial sleep onset in primary insomnia: a case-control study using 4seconds epochs. J Clin Sleep Med $\underline{3}$ (5), 479-488

Mufson LH, Moreau D, Weissman MM, Klerman GL: Interpersonal psychotherapy for adolescent depression; in: New applications of interpersonal psychotherapy; hrsg. v. Klerman GL, Weissman MM; American Psychiatric Press, Washington DC 1993, 129 166

Müller-Popkes K: Interpersonelle Psychotherapie zur Behandlung von Patienten mit primärer Insomnie: Ein Vergleich mit progressiver Muskelrelaxation. Cuvillier-Verlag, Göttingen 1997 (zugl.: Diss., Kiel 1997)

Murtagh DRR and Greenwood KM (1995): Identifying effective psychological treatments for insomnia: a meta-analysis. J Consult Clin Psychol $\underline{63}$, 79-89

Nau SD, Walsh JK (1983): Sleep hygiene of insomnia patients. Sleep Res 12, 268

Nicassio P and Bootzin R (1974): A comparison of progressive relaxation and autogenic training as treatments for insomnia. J Abnorm Psychol 조, 253-260

Nicassio P, Boylan MB and McCabe TG (1982): Progressive relaxation, EMG biofeedback and biofeedback placebo in the treatment of sleep-onset insomnia. $\mathrm{Br} \mathrm{J}$ Med Psychol 포, 159-166

Nieuwenhuijs DJ (2006): Processed EEG in natural sleep. Best practice \& research. Clin Anaesthesiol $\underline{20}$ (1), 49-56

Norman RG, Pal I, Stewart C, Walsleben JA, Rapoport DM (2001): Interobserver agreement among sleep scorers from different centers in a large dataset. Sleep $\underline{23}$ (7), 901-908

Nowell PD, Buysse DJ, Reynolds CF III, Hauri P, Roth T, Stephanski EJ, Thorpy MJ, Bixler E, Kales A, Manfredi RL, Vgontzas AN, Stapf DM, Houck PR, Kupfer DJ (1997a): Clinical factors contributing to the differential diagnosis of primary insomnia and insomnia related to mental disorders. Am J Psychiatry 154 (10), 1412-1416

Nowell PD, Mazumdar S, Buysse DJ, Dew MA, Reynolds CF III, Kupfer DJ (1997b): Benzodiazepines and zolpidem for chronic insomnia. A meta-analysis of treatment efficacy. JAMA 278 (24), 2170-2177 
Ott H, Oswald I, Fichte K und Sastre-Y-Hernandez M: Visuelle Analogskalen zur Erfassung von Schlafqualität (VIS-A und VIS-M). Selbstbeurteilungsskala (S); in: Internationale Skalen für Psychiatrie; hrsg. v. Collegium Internationale Psychiatriae Scalarum (CIPS); Beltz, Weinheim 1981, o.Sz.

Partinen M, Kaprio J, Koskenvuo M, Putkonen P, Langinvainio H (1983): Genetic and environmental determination of human sleep. Sleep $\underline{6}$, 179-185

Paterok B: Gruppentherapie bei primären Schlafstörungen: Effektivität eines multifaktoriellen Ansatzes. Lit Verlag, Münster 1993 (zugl.: Diss., Bielefeld 1993)

Penzel T, Hajak G, Hoffmann M, Lund R, Podszus T, Pollmächer T, Schäfer T, Schulz H, Sonnenschein W, Spieweg I (1993): Empfehlungen zur Durchführung und Auswertung polygraphischer Ableitungen im diagnostischen Schlaflabor. Z EEG EMG $\underline{24}, 65-70$

Pillar G, Betito M, Schnall RP, Dvir I, Sheffy J, Lavie P (2003): An automatic ambulatory device for detection of AASM defined arousals from sleep: the WP 100 . Sleep Med $\underline{4}$ (3), 207-212

Polo-Kantola P, Erkkola R, Helenius H, Irjala K, Polo O (1998): When does estrogen replacement therapy improve sleep quality? Am J Obstet Gynecol 178 (5), 1002-1009

Pritz A: Kurzgruppenpsychotherapie. Struktur, Verlauf und Effektivität von autogenem Training, progressiver Muskelentspannung und analytisch fundierter Kurzgruppenpsychotherapie. Springer, Berlin 1990

Rechtschaffen A: Polygraphic aspects of insomnia; in: The abnormalities of sleep in man; hrsg. v. Gastaut H, Lugaresi E, Berti-Ceroni G; Gaggi, Bologna 1968, 109-125

Rechtschaffen A, Kales A: A manual of standardized terminology, techniques and scoring system for sleep stages of human subjects; hrsg. v. National Institute of Health, Bethesda MD 1968

Ree MJ, Pollitt A, Harvey AG (2006): An investigation of interpretive bias in insomnia: an analog study comparing normal and poor sleepers. Sleep $\underline{29}$ (10), 1359-1362

Regestein QR, Dambrosia J, Hallet M, Murawski B, Paine M (1993): Daytime-alertness in patients with primary insomnia. Am J Psychiatry 150 (10), 1529-1534 
Riedel BW, Lichstein KL, Peterson BA, Epperson MT, Means MK, Aguillard RN (1998): A comparison of the efficacy of stimulus control for medicated and nonmedicated insomniacs. Behav Modif 22 (1), 3-28

Rombaut N, Maillard F, Kelly F and Hindmarch I (1990): The quality of life of insomniacs questionnaire (QOLI). Med Sci Res 18, 845-847; (deutsche Übersetzung: Müller-Popkes K: Fragebogen zur Lebensqualität; in: Interpersonelle Psychotherapie zur Behandlung von Patienten mit primärer Insomnie: Ein Vergleich mit progressiver Muskelrelaxation. Cuvillier-Verlag, Göttingen 1997; Anhang)

Rounsaville BJ, Glazer W, Wilber CH (1983): Short-term interpersonal psychotherapy in methadone-maintained opiate addicts. Arch Gen Psychiatry $\underline{40}$, 629-636

Rounsaville BJ, Carroll K: Interpersonal psychotherapy for patients who abuse drugs; in: New applications of interpersonal psychotherapy; hrsg. v. Klerman GL, Weissman MM; American Psychiatric Press, Washington DC 1993, 319-352

Russel GFM (1979): Bulimia nervosa: an ominous variant of anorexia nervosa. Psychol Med $\underline{9}, 429-448$

Saletu-Zyhlarz G, Anderer P, Brandstätter N, Dantendorfer K, Gruber G, Mandl M, Ritter K, Zoghlami A, Saletu B (2000): Placebo-controlled sleep laboratory studies on the acute effects of zolpidem on objective and subjective sleep and awakening quality in nonorganic insomnia related to neurotic and stress-related disorder. Neuropsychobiology $\underline{41}$ (3), 139-148

Salin-Pascual RJ, Roehrs TA, Merlotti LA, Zorick F, Roth T (1992): Long-term study of the sleep of insomnia patients with sleep state misperception and other insomnia patients. Am J Psychiatry $\underline{149}$ (7), 904-908

Schiff A (2006): Rückenmassage und verwandte Techniken zur Förderung des Schlafes bei älteren Menschen: Eine Literaturanalyse. Pflege 19 (3), 163-173

Schoicket SL, Bertelson AD, Lacks P (1988): Is sleep hygiene a sufficient treatment for sleep maintenance insomnia? Behav Ther $\underline{19}$, 183-190

Schramm E: Psychodiagnostische Erfassung von Schlafstörungen; in: Handbuch des normalen und gestörten Schlafs; hrsg. v. Berger M; Springer, Berlin 1992, 45-66 
Schramm E: Modifizierte Version der Interpersonellen Psychotherapie zur Behandlung von Insomnie (IPT-I). Manual; Freiburg 1993, unveröffentlicht; zit. n. Müller-Popkes 1997, 8, 58, 69-70

Schramm E (2007): Interpersonelle Psychotherapie bei affektiven Störungen. Psychiatr Psychother up2date $\underline{1}$, 357-365

Schramm E, Berger M (1994): Zum gegenwärtigen Wissensstand der interpersonellen Psychotherapie. Nervenarzt $\underline{65}$ (1), 2-10

Schramm E, Hohagen F, Graßhoff U, Riemann D, Hajak G, Weeß H-G, Berger M: Testretest-reliability of a structured interview for sleep disorders according to DSM-III-R (SIS-D); in: Sleep; hrsg. v. Horne J; Pontenagel Press, Göttingen 1990

Schramm E, Hohagen F, Graßhoff U, Berger M: Strukturiertes Interview für Schlafstörungen nach DSM III-R. Beltz, Weinheim 1991

Schultz JH, Luthe W: Autogenic training. A psychophysiological approach in psychotherapy. Grune and Statton, New York 1959

Shear K, Frank E, Houck PR, Reynolds CF $3^{\text {rd }}$ (2005): Treatment of complicated grief: a randomized controlled trial. JAMA 293 (21), 2601-2608

Sholomskas AJ, Chevron ES, Prusoff BA (1983): Short-term interpersonal therapy (IPT) with the depressed elderly: case reports and discussion. Am J Psychother 37, 552566

Sievertsen B, Nordhus IH (2007): Management of insomnia in older adults. $\mathrm{Br} \mathrm{J}$ Psychiatry 190, 285-286

Simen S, Hajak G, Schlaf G, Westenhöfer J, Rodenbeck A, Bandelow B, Pudel V und Rüther E. (1995): Chronifizierung von Schlafbeschwerden - Ergebnisse einer Repräsentativumfrage. Nervenarzt $\underline{66}$, 686-695

Simen S, Rodenbeck A, Schlaf G, Müller-Popkes K, Hajak G (1996): Schlafbeschwerden und Schlafmitteleinnahme im Alter- Ergebnisse einer Repräsentativumfrage in Westdeutschland. Wien Med Wochenschr 13/14, 306-309

Soares CN, Murray BJ (2006) Sleep disorders in woman: clinical evidence and treatment strategies. Psychiatr Clin North Am $\underline{29}$ (4), 1095-1113 
Spielman AJ, Caruso LS, Glovinsky PB (1987): A behavioral perspective on insomnia treatment. Psychiatr Clin North Am 10 (4), 541-553

Steinberg R, Brenner PM, Lund R, Rüther E: Behandlung chronischer Schlafstörungen; in: Schlaf-Wach-Funktionen; hrsg. v. Hippius H, Rüther E, Schmauß M; Springer, Berlin 1987, 131-143

Steinmark SW, Borkovec TD (1974):Active and placebo treatment effects on moderate insomnia under counterdemand and positive demand instructions. J Abnorm Psychol $\underline{\text { 33, }}$ 157-163

Stepanski E, Lamphere J, Badia P, Zorick F, Roth T (1984): Sleep fragmentation and daytime sleepiness. Sleep $\underline{7}, 18-26$

Stepanski EJ (2005): Hypnotics should not be considered for the initial treatment of chronic insomnia. Con. J Clin Sleep Med 151 (2), 125-128

Sullivan HS: The interpersonal theory of psychiatry. WW Norton, New York 1953

Trilling JS (1992): A problem-solving approach to the treatment of insomnia: selected case histories. J Clin Psychiatry $\underline{53}$ (6) (suppl), 42-45

Trinder J (1988): Subjective Insomnia without objective findings: A pseudodiagnostic classification? Psychol Bull $\underline{103}$, 87-94

Turner RM and Ascher LM (1979): Controlled comparison of progressive relaxation, stimulus control, and paradoxical intention therapies for insomnia. J Consult Clin Psychol 47, 500-508

Vein AM, Sudakow KV, Levin YI, Yumatov EA, Strygin KN, Kovrov GV (2002): Stages of sleep after psychoemotional tension: the individual character of changes. Neurosci Behav Physiol $\underline{32}$ (5), 513-518

Vgontzas AN, Kales A (1999): Sleep and its disorders. Annu Rev Med 50, 387-400

Vollrath M, Wicki W, Angst J (1989): The Zurich study: VIII. Insomnia: association with depression, anxiety, somatic syndromes, and course of insomnia. Eur Arch Psychiatry Neurol Sci 239, 113-124

Walsh JK, Schweitzer PK (1999): Ten-year trends in the pharmacological treatment of insomnia. Sleep 22 (3), 371-375 
Weissman MM, Klerman GL: Conjoint interpersonal psychotherapy for depressed patients with marital disputes; in: New applications of interpersonal psychotherapy; hrsg. v. Klerman GL, Weissman MM; American Psychiatric Press, Washington DC 1993, 103-127

Weissman MM, Prusoff BA, DiMascio A, Neu C, Goklaney M, Klerman GL (1979): The efficacy of drugs and psychotherapy in the treatment of acute depressive episodes. Am J Psychiatry 136, 555-558

Weissman MM, Greenwald S, Nino-Murcia G, Dement WC (1997): The morbidity of insomnia uncomplicated by psychiatric disorders. Gen Hosp Psychiatry $\underline{19}$ (4), 245-250

Westermann R, Hager W (1982): Entscheidung über statistische und wissenschaftliche Hypothesen: Zur Differenzierung und Systematisierung der Beziehungen. Z Sozialpsychol $\underline{13}, 13-21$

WHO = Weltgesundheitsorganisation: Internationale Klassifikation psychischer Störungen ICD-10. Kapitel V (F). Klinisch-diagnostische Leitlinien; hrsg. v. Dilling H, Mombour W, Schmidt MH; Huber, Bern 1991

WHO = Weltgesundheitsorganisation: Taschenführer zur ICD-10-Klassifikation psychischer Störungen; hrsg. v. Dilling H, Freyberger HJ. 4. überarb. Aufl. unter Berücksichtigung der German Modification (GM) der ICD-10. Huber, Bern 2008 (nach dem englischsprachigen Pocket Guide von J.E. Cooper; Churchill Livingstone, Edinburgh, London, Melbourne, New York, Tokyo 1994)

Williams RL, Karacan I, Hursch CJ: Electroencephalography of human sleep: Clinical applications. Wiley, New York 1974

Wohlgemuth WK, Edinger JD, Fins AI, Sullivan RJ Jr (1999): How many nights are enough? The short-term stability of sleep parameters on elderly insomniacs and normal sleepers. Psychophysiology $\underline{36}$ (2), 233-244

Wolpe J: Psychotherapy by reciprocal inhibition. Stanford University Press, Stanford CA 1958

Woody GE, Luborsky L, McLellan AT (1983): Psychotherapy for opiate addicts: does it help? Arch Gen Psychiatry 40, 639-645

Woolfolk RL, McNulty TF (1983): Relaxation treatment for insomnia: a component analysis. J Consult Clin Psychol $\underline{51}$, 495-503 
Zerssen D von: Paranoid-Depressivitäts-Skala. Parallelformen PD-S und PD-S'. Depressivitäts-Skala. Parallelformen D-S und D-S'. Beltz, Weinheim 1976 


\section{Danksagung}

Allen voran danke ich Herrn Professor Dr. Göran Hajak für die Überlassung des Themas und die sehr kompetente Betreuung. Mit seiner Freundlichkeit, seinem Wohlwollen und seiner Kritik hat er sehr zur Fertigstellung dieser Arbeit beigetragen.

Herrn Prof. Dr. E. Rüther bin ich sehr dankbar, dass ich an der klinischen Untersuchung mitarbeiten konnte, für die er die erste Idee hatte.

Ganz besonders danke ich Frau Dr. Kristin Müller-Popkes. Sie hat mit großem Einfühlungsvermögen die Therapien durchgeführt, mir in statistischen Fragen beigestanden und die Dissertation betreut. Ständig stand sie für Fragen und bei Problemen zur Verfügung und bot mit ihrer psychologischen Prägung stets konstruktive Kritik.

Allen Mitarbeiterinnen und Mitarbeitern des Schlaflabors der Abteilung Psychiatrie danke ich sehr für ihre Hilfe, ohne die mir die Auswertung der Schlafableitungen nicht möglich und der Zugang zur Literatur erschwert gewesen wäre. Meiner Kommilitonin Stefanie Kesten danke ich herzlich für die sehr gute Zusammenarbeit in der Patientenbetreuung und der Datenerhebung. 